\title{
Rocket Based Combined Cycle Exchange Inlet Performance Estimation at Supersonic Speeds
}

\author{
by
}

\section{Aliaksandr Murzionak}

\author{
A Thesis submitted to \\ the Faculty of Graduate Studies and Research \\ in partial fulfilment of \\ the requirements for the degree of \\ Master of Applied Science \\ in
}

\author{
Aerospace Engineering \\ Department of Mechanical and Aerospace Engineering \\ Carleton University \\ Ottawa, Ontario, Canada \\ January 2013
}

Copyright (C)

2013 - Aliaksandr Murzionak 
Library and Archives

Canada

Published Heritage

Branch

395 Wellington Street

Ottawa ON K1A ON4

Canada
Bibliothèque et

Archives Canada

Direction du

Patrimoine de l'édition

395 , rue Wellington

Ottawa ON K1A ON4

Canada
Your file Votre référence

ISBN: 978-0-494-94260-4

Our file Notre référence

ISBN: 978-0-494-94260-4
NOTICE:

The author has granted a nonexclusive license allowing Library and Archives Canada to reproduce, publish, archive, preserve, conserve, communicate to the public by telecommunication or on the Internet, loan, distrbute and sell theses worldwide, for commercial or noncommercial purposes, in microform, paper, electronic and/or any other formats.

The author retains copyright ownership and moral rights in this thesis. Neither the thesis nor substantial extracts from it may be printed or otherwise reproduced without the author's permission.
AVIS:

L'auteur a accordé une licence non exclusive permettant à la Bibliothèque et Archives Canada de reproduire, publier, archiver, sauvegarder, conserver, transmettre au public par télécommunication ou par l'Internet, prêter, distribuer et vendre des thèses partout dans le monde, à des fins commerciales ou autres, sur support microforme, papier, électronique et/ou autres formats.

L'auteur conserve la propriété du droit d'auteur et des droits moraux qui protege cette thèse. $\mathrm{Ni}$ la thèse ni des extraits substantiels de celle-ci ne doivent être imprimés ou autrement reproduits sans son autorisation.
In compliance with the Canadian Privacy Act some supporting forms may have been removed from this thesis.

While these forms may be included in the document page count, their removal does not represent any loss of content from the thesis.
Conformément à la loi canadienne sur la protection de la vie privée, quelques formulaires secondaires ont été enlevés de cette thèse.

Bien que ces formulaires aient inclus dans la pagination, il n'y aura aucun contenu manquant. 


\section{Abstract}

A method to estimate the performance of an exchange inlet for a Rocket Based Combined Cycle engine is developed. This method is to be used for exchange inlet geometry optimization and as such should be able to predict properties that can be used in the design process within a reasonable amount of time to allow multiple configurations to be evaluated. The method is based on a curve fit of the shocks developed around the major components of the inlet using solutions for shocks around sharp cones and $2 \mathrm{D}$ estimations of the shocks around wedges with blunt leading edges. The total pressure drop across the estimated shocks as well as the mass flow rate through the exchange inlet are calculated. The estimations for a selected range of free-stream Mach numbers between 1.1 and 7 are compared against numerical finite volume method simulations which were performed using available commercial software (Ansys-CFX). The total pressure difference between the two methods is within $10 \%$ for the tested Mach numbers of 5 and below, while for the Mach 7 test case the difference is $30 \%$. The mass flow rate on average differs by less than $5 \%$ for all tested cases with the maximum difference not exceeding $10 \%$. The estimation method takes less than 3 seconds on $3.0 \mathrm{GHz}$ single core processor to complete the calculations for a single flight condition as oppose to over 5 days on 8 cores at $2.4 \mathrm{GHz}$ system while using 3D finite volume method simulation with 1.5 million elements mesh. This makes the estimation method suitable for the use with exchange inlet geometry optimization algorithm. 


\section{Acknowledgments}

I would like to thank my supervisor professor J. Etele for guiding me towards the completion of this work. I would also like to thank my colleagues for providing me with good advices.

I am very grateful to my family who were always supportive through my years of education and who made this work possible. A special thanks to my brother, who, despite his tight schedule at the time, managed to thoroughly go through this thesis and provide with constructive feedback. 


\section{Table of Contents}

$\begin{array}{ll}\text { Abstract } & \text { ii }\end{array}$

$\begin{array}{ll}\text { Acknowledgments } & \text { iii }\end{array}$

Table of Contents $\quad$ iv

List of Tables $\quad$ vi

List of Figures $\quad$ vii

List of Acronyms $\quad$ x

List of Symbols $\quad$ xi

1 Introduction $\quad 1$

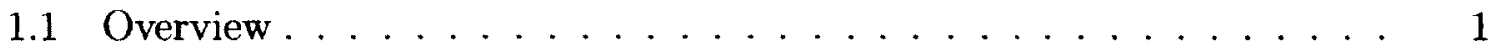

$1.2 \mathrm{RBCC} \ldots \ldots \ldots \ldots \ldots \ldots \ldots$

1.3 Problem Statement . . . . . . . . . . . . . . . 9

1.4 Computational Methods .................. 10

1.4.1 Navier-Stokes Solver . . . . . . . . . . . . . . 11

1.4.2 Method of Characteristics . . . . . . . . . . . . 12

1.4.3 Semi-Analytical Method .............. 13

2 Exchange Inlet Geometry $\quad 15$ 


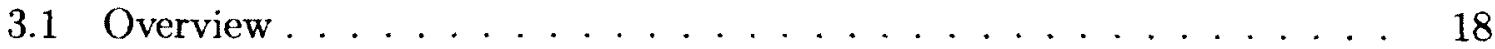

3.2 Isentropic compressible flow and oblique shock equations . . . . . . . 21

3.3 Centre Body Shock Estimation . . . . . . . . . . . . . 25

3.4 Fairings Shock Estimation . . . . . . . . . . . . . . . 28

3.5 Cowl Shock Estimation . . . . . . . . . . . . . . 35

3.6 Mass Flow Rate Estimation . . . . . . . . . . . . . . . . 46

4 2D CFD simulations $\quad 49$

$4.12 \mathrm{D}$ geometry . . . . . . . . . . . . . . . . . . 49

4.2 Domain, Mesh and Simulation setup . . . . . . . . . . 50

4.3 Grid Sensitivity Study . . . . . . . . . . . . . 55

4.4 Results and comparison ..................... 58

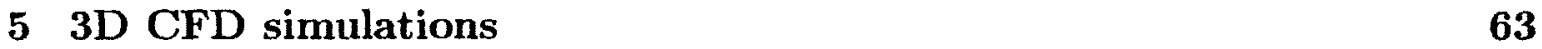

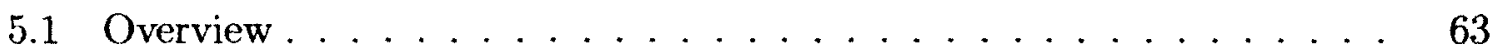

$5.23 \mathrm{D}$ Simulation Setup . . . . . . . . . . . . . . 63

5.3 Grid sensitivity analysis . . . . . . . . . . . . . . 69

5.4 Results and comparison .................. 72

5.4 .1 Shock geometry ................... 72

5.4 .2 Total pressure . . . . . . . . . . . . . 79

5.4 .3 Mass flow rate ..................... 82

6 Conclusions and Recommendations $\quad 85$

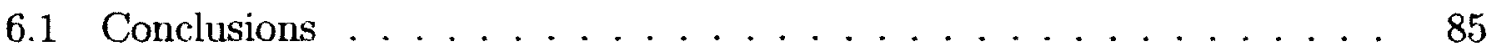

6.2 Recommendations ................... 86

$\begin{array}{ll}\text { List of References } & \mathbf{8 8}\end{array}$ 


\section{List of Tables}

3.1 Summary of angles used for the shock-expansion wave interaction calculations. ....................... 44

4.1 List of meshes for $2 \mathrm{D}$ simulations. . . . . . . . . . . . 55

4.2 Grid convergence summary. . . . . . . . . . . . . 55

5.1 Flight Conditions. . . . . . . . . . . . . . 66

5.2 List of meshes for 3D grid convergence study . . . . . . . . . . . . 69

5.3 Grid convergence summary. . . . . . . . . . . . . 71

5.4 Pressure ratio difference. . . . . . . . . . . . . . . 81

5.5 Mass flow rate percent difference. . . . . . . . . . . . . . . . 83 


\section{List of Figures}

1.1 Approximate specific impulse performance of different propulsion cycles (modified from [1]. with additional infornation from [2]) . . . . 2

1.2 Schematic of an RBCC engine. . . . . . . . . . 4

1.3 Modes of operation of an $\mathrm{RBCC}$ engine. . . . . . . . . . 5

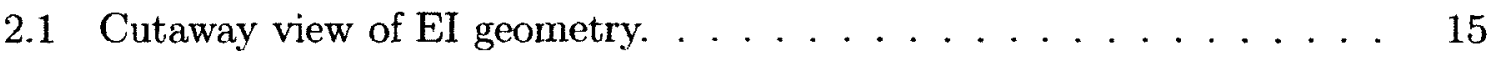

2.2 Definition of half-section of the EI and the svmmetry planes. . . . . 17

2.3 Section of EI geometry as imported into the estimation code. . . . . 17

3.1 Main shocks generated by the geometry . . . . . . . . . . . 19

3.2 Supersonic expansion around a corner. . . . . . . . . . 22

3.3 Oblique shock nomenclature . . . . . . . . . . . . . . . . 23

3.4 Shock generated by a cone at zero angle of attack. . . . . . . . 26

3.5 Cone shock with non-dimensional velocity components shown. . . . 26

3.6 Cone shock angle dependence on the cone half-angle and the free stream Mach number. . . . . . . . . . . . . . 27

3.7 Schematic of cone shock generated by a centre body. . . . . . . 28

3.8 Shock generated by the fairing. . . . . . . . . . . . . . 29

3.9 Shock generated by a wedge. . . . . . . . . . . . . . . . 29

3.10 Variables used to determine detached shock geometries. . . . . . . 31

3.11 Shock generated by the fairing with shock-plane shown. . . . . . . 34

3.12 Flow acceleration around the fairing. . . . . . . . . 35 
3.13 Possible reflection shocks. $\ldots \ldots \ldots \ldots \ldots \ldots$

3.14 Variables required for Mach reflection analysis. . . . . . . . . . 38

3.15 Mach reflection. . . . . . . . . . . . . . . . . 40

3.16 Side view of cowl shock structure . . . . . . . . . . . 41

3.17 Shock/Expansion wave intersection (numbers in brackets indicate the regions shown in Figure 3.16 from previous analysis). . . . . . . . 43

3.18 Variables needed for the calculation of the mass flow rate through EI. 46

3.19 Half-section with the variables for area calculations. . . . . . . . . 47

4.1 Simple 2D geometry. . . . . . . . . . . . . . . . . . . 49

$4.22 \mathrm{D}$ domain with boundary conditions shown $(\mathrm{R}=0.1 \mathrm{~m}) . \ldots \ldots$

4.3 Examples of the mesh for 2D CFD simulations $(R=0.01 \mathrm{~m}) \ldots \ldots 52$

4.4 Mach gradient distribution along the symmetry plane for different mesh $\operatorname{sizes}\left(N_{\infty}=1.1\right) \ldots \ldots \ldots \ldots \ldots \ldots \ldots \ldots \ldots \ldots \ldots \ldots \ldots \ldots$

4.5 Plot of Residuals $\left(M_{\infty}=2.0\right) \ldots \ldots \ldots \ldots \ldots \ldots$

4.6 Stand-off distance as a function of the mesh size. $\ldots \ldots \ldots$

4.7 Mach distribution along the symmetry plane. . . . . . . . . 57

4.8 Mach gradient distribution along the symmetry plane $\left(\Lambda_{\infty}=2.0\right) \ldots \quad 57$

4.9 Shock stand-off distance vs Mach number. . . . . . . . . . . 58

4.10 Shape of the shocks produced by a blunt body at different Mach numbers $(k=0.1 \mathrm{~m}) \ldots \ldots \ldots \ldots \ldots \ldots \ldots \ldots \ldots \ldots \ldots$

4.11 Shape of the shocks produced by a blunt body at different Mach num-

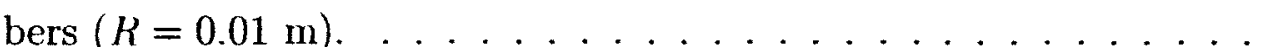

4.12 Shape of the shocks produced by a blunt body at different Mach numbers, for CFD $\theta=0 \mathrm{deg}$, while for Billig and Estimations $\theta=3 \mathrm{deg}$ $(R=0.01 \mathrm{~m}) \ldots \ldots \ldots \ldots \ldots \ldots \ldots \ldots \ldots$

4.13 Shape of the shocks produced by a blunt body with different deflection angles $\left(R=0.01 \mathrm{~m}, M_{\infty}=2.0\right) \ldots \ldots \ldots \ldots \ldots \ldots$ 
4.14 Contour plot of the flow direction for $\left(R=0.01 \mathrm{~m}, M_{\infty}=2.0\right) \ldots \ldots$

5.1 Half-section of the EI used for 3D simulations with symmetry planes shown. . . . . . . . . . . . . . . . . 64

5.2 The domain setup for CFD simulations with boundaries shown. . . . 64

5.3 Centre body tip geometry. . . . . . . . . . . . . . 65

5.4 Simulated flight profile. . . . . . . . . . . . . . 65

5.5 Plot of Residuals $\left(M_{\infty}=2.0\right) \ldots \ldots \ldots \ldots \ldots \ldots$

5.6 Test locations for the time and grid convergence checks. . . . . . . 67

5.7 Time Convergence (Coarse Mesh, $\left.M_{\infty}=2.0\right) \ldots \ldots \ldots 6$

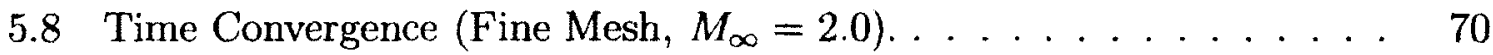

5.9 Grid Convergence. . . . . . . . . . . . . . . 72

5.10 Shock geometries obtained from 3D CFD simulations. . . . . . . . 73

5.11 Cowl shock $\left(M_{\infty}=7\right) \ldots \ldots \ldots \ldots \ldots \ldots \ldots$

5.12 Mach distribution (Symmetry Plane 1) with lines representing the estimated shocks. . . . . . . . . . . . . . . 76

5.13 Fairing shock location and its projection to Symmetry Plane 1. . . . 77

5.14 Mach distribution after the fairing shock (Selected Planes) . . . . . 78

5.15 Mach number along the centre body surface (estimation line ends at the location of the fairing shock $) \ldots \ldots \ldots \ldots 79$

5.16 Pressure loss through the intake with cumulative total pressure after each shock shown. . . . . . . . . . . . . . . 79

5.17 Example of averaging locations for total pressure comparison $\left(M_{\infty}=\right.$

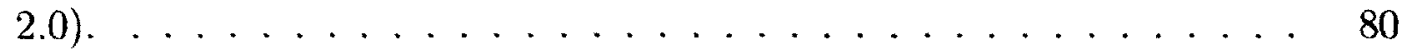

5.18 Pressure ratio difference. . . . . . . . . . . . . . . 82

5.19 Estimations of the mass flow rate . . . . . . . . . . . 83

5.20 Mass flow rate comparison. . . . . . . . . . . . . . 84

5.21 Mass flow rate percent difference. . . . . . . . . . . . 84 


\section{List of Acronyms}

\begin{tabular}{ll}
\hline Acronyms & Definition \\
\hline CFD & Computation Fluid Dynamics \\
DAB & Diffusion and Afterburning \\
EI & Exchange Inlet \\
FVM & Finite Volume Method \\
HR & High Resolution advection scheme \\
ODE & Ordinary Differential Equation \\
RBCC & Rocket Based Combined Cycle \\
RMS & Root Mean Square \\
SMC & Simultaneous Mixing and Combustion \\
wrt & with respect to \\
\hline
\end{tabular}




\section{List of Symbols}

\begin{tabular}{|c|c|}
\hline Symbols & Definition \\
\hline$a$ & $\begin{array}{l}\text { Distance from the coordinate origin to the vertex of a hy- } \\
\text { perbole }[\mathrm{m}]\end{array}$ \\
\hline$d_{s}$ & Shock stand-off distance $[\mathrm{m}]$ \\
\hline$\dot{m}$ & Mass flow rate $[\mathrm{kg} / \mathrm{s}]$ \\
\hline$r$ & Radial position [m] \\
\hline$v$ & Non-dimensional velocity component \\
\hline$x$ & $\mathrm{x}$ coordinate $[\mathrm{m}]$ \\
\hline$y$ & y coordinate $[\mathrm{m}]$ \\
\hline$A$ & Area $\left[\mathrm{m}^{2}\right]$ \\
\hline$M$ & Mach number \\
\hline$P$ & Static pressure $[\mathrm{Pa}]$ \\
\hline$P_{o}$ & Total Pressure $[\mathrm{Pa}]$ \\
\hline$R$ & Radius $[\mathrm{m}]$ \\
\hline
\end{tabular}




\begin{tabular}{|c|c|}
\hline$K_{a u r}$ & Gas constant (air) $[\mathrm{J} / \mathrm{kg} / \mathrm{K}]$ \\
\hline$T$ & Static Temperature $[\mathrm{K}]$ \\
\hline$T_{0}$ & Total Temperature $[\mathrm{K}]$ \\
\hline$V$ & Velocity $[\mathrm{m} / \mathrm{s}]$ \\
\hline $\bar{V}$ & Non-dimensional velocity \\
\hline$\beta$ & Shock angle [rad] \\
\hline$\gamma$ & Ratio of specific heats \\
\hline$\epsilon$ & Eccentricity of a hyperbole \\
\hline$\nu$ & Prandtl-Meyer function \\
\hline$\theta$ & Flow deflection angle / Flow direction angle $[\mathrm{rad}]$ \\
\hline$\rho$ & Density $\left[\mathrm{kg} / \mathrm{m}^{3}\right]$ \\
\hline$\tau$ & Mach wave angle [rad] \\
\hline$\psi$ & $\begin{array}{l}\text { Angle of the ray of constant properties for flows around } \\
\text { cones [rad] }\end{array}$ \\
\hline
\end{tabular}

\section{Superscript}

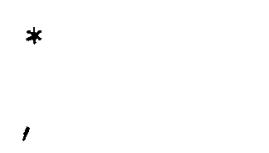

Throat/choked conditions

Direction with respect to horizontal line

\section{Subscript}

Conditions before a shock 


\begin{tabular}{|c|c|}
\hline 2 & Conditions after a shock \\
\hline c & Cone \\
\hline$c b$ & Centre body \\
\hline$c w$ & Cowl \\
\hline$d$ & Near-detached shock conditions \\
\hline$i$ & Incident shock \\
\hline$n$ & Normal component of velocity/Mach \\
\hline$o$ & Stagnation conditions \\
\hline$r$ & Reflected shock / radial component \\
\hline$t$ & Tangential component of velocity/Mach \\
\hline$s$ & Shock \\
\hline$s s$ & Slipstream \\
\hline$w$ & Wedge \\
\hline$\infty$ & Free stream conditions \\
\hline$F F$ & Flow Field \\
\hline
\end{tabular}




\section{Chapter 1}

\section{Introduction}

\subsection{Overview}

For more than 50 years humans have been launching satellites into space. Currently, the only means available for space launch applications are rockets. Rockets carry both the fuel and the oxidiser on board, and as such they can operate in atmosphere as well as in space. However, carrying the oxidiser on board is what makes the rockets heavy and less efficient than air breathing engines. In addition to being heavy, most of the rockets for space launch applications are not reusable. The exceptions to this are some of the partially reusable systems such as the US Space Shuttle system (now retired), X-37 [3]. and Soviet space system "Energia-Buran" (flew only once into space) [4]. In addition to these vehicles there were a number of vehicles for testing the gliding descent from space (US: M2, X-20 [3]; USSR: BOR [4], [5]). Over time it became apparent that these reusable systems are even more expensive than nonreusable, mainly due to the maintenance. This, however, does not stop research and development of reusable space launch systems. Currently, there are a few projects that try to make reusable or partially reusable space launchers more appealing from the financial point of view. Reusable Falcon 9 by SpaceX, and reusable boosters for Angara by Khrunichev State Research and Production Space Center are just a few 
examples of such systems. These new vehicles use the traditional rocket engines, with improved performance and better materials, which can potentially reduce the cost of space launch, however, at this point they do not address the issue of the weight of the oxidiser on board, which is still being carried by the vehicle.

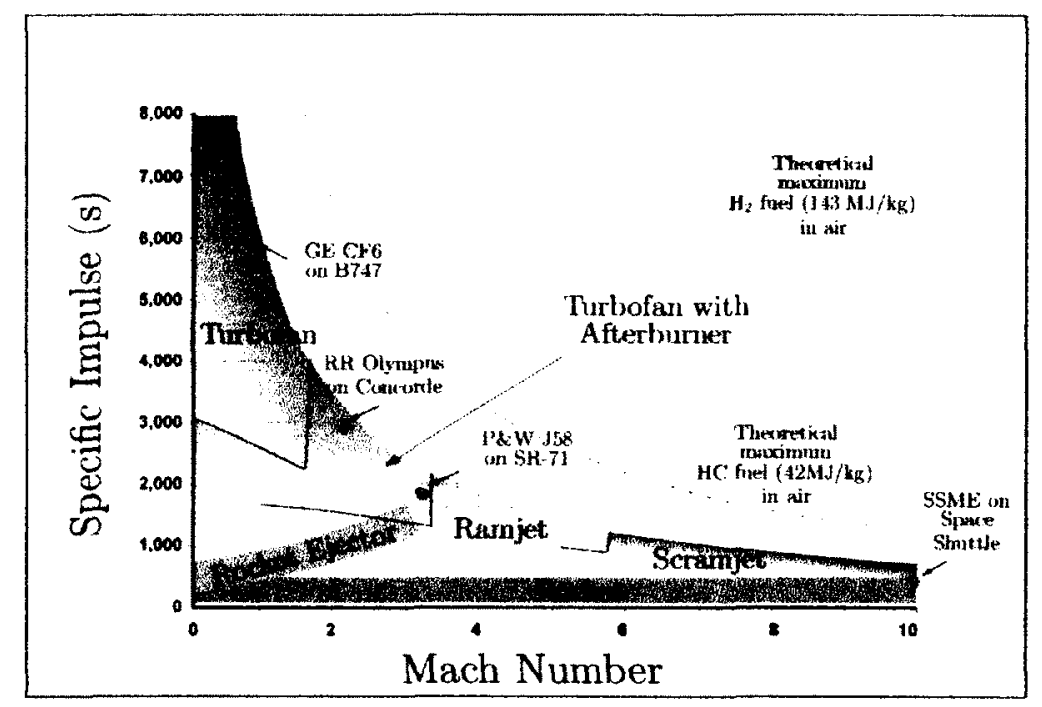

Figure 1.1: Approximate specific impulse performance of different propulsion cycles (modified from [1], with additional information from [2]).

The idea of not carrying fuel at all in the space launch vehicle also exists and includes such concepts as space elevator and ground based power systems (laser/microwave powered propulsion). The space elevator currently lacks the materials of required strength, while the vehicles powered from the ground by means of laser or microwave run into the issue of laser/microwaves absorption by air or dissipation. This means that at least for now chemical powered vehicles are the only way to go. Figure 1.1 shows the specific impulse comparison of different chemical engines. As seen from the figure the turbojet engines have higher specific impulse as opposed to the rocket engines, meaning lower fuel consumption for the same amount of thrust, therefore lighter overall vehicle in the case of the air breathing engine. This leads to an idea of using an aircraft as a first stage of a space launch system in which case a rocket is lifted to high altitude by an aircraft with high performance air 
breathing engines. Examples include Pegasus [6], SpaceShipOne and SpaceShipTwo. In these cases large spaceships and satellites would require a large carrier design. An alternative to an aircraft dropped rockets is combined cycle propulsion, which uses different modes of operation (shown in Figure 1.1) along the flight trajectory improving the overall specific impulse of the vehicle potentially reducing the overall weight, while increasing the payload fraction as compared to rocket-only configuration. The combined cycle vehicles seem to be promising with the current level of technology and were previously extensively analysed [7], [8], and [9]. A few of these engines are currently under active development, namely Skylon [10]. Combined cycle engines are just that, they combine two or more modes of operation within the same engine, one of which conld be a rocket mode for space flight, while other modes are air-breathing types. There are mainly two of them that are under consideration: Rocket-BasedCombined-Cycle (RBCC) and Turbine-Based-Combined-Cycle (TBCC). A TBCC is build around a turbojet engine, which operates from start to low supersonic speeds, then switches to ramjet with a possibility of a switch to scramjet mode of operation at higher Mach numbers. Once the engine reaches the altitude where airbreathing modes of operation are not viable the TBCC engine is operated as a rocket. Figure 1.1 indicates that all of the modes used by the TBCC have higher specific inuulse, when compared to rocket engines, and as such have potential in the reduction of the weight of the entire vehicle, while increasing the weight portion of the payload carried by such vehicle. The best example of the TBCC engine is the Skylon project with the SABRE (Synergistic Air-Breathing Rocket Engine) engine [10]. Another example is a French Griffon II aeroplane build in the 1957 [9]: which means that the idea of the TBCC engines is not new. In the case of Griffon II. the aircraft only operated in turbojet and ramjet modes without the need for a rocket engine since it was not meant for space flights. The RBCC on the other hand uses ejector mode of operation instead of turbojet engine while all other modes of operations are still possible. 
This work is concentrated mainly on the RBCC and the design of an Exchange Inlet (EI) for improved performance. The next section describes the RBCC engines in more detail.

\subsection{RBCC}

In this work an RBCC engine is defined as an engine which combines within the same housing the rocket engine with an air breathing engine. This means they use common components and common flow paths. Figure 1.2 shows a schematic of an RBCC engine. The RBCC engine consists of a rocket engine with the rocket nozzle directing the rocket exhaust into the mixing duct. In the design currently being developed at Carleton University, the air comes through air passages within the exchange inlet, which also houses the rocket engine. In Figure 1.2 the rocket exhaust is shown to be located at the outer radius of the mixing duct. This is just one configuration among

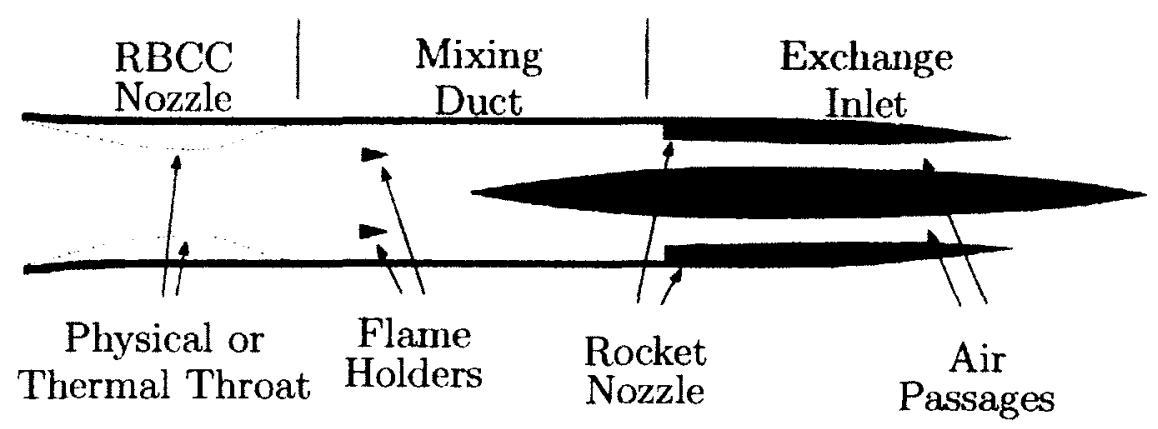

Figure 1.2: Schematic of an RBCC engine.

many being analysed by many authors. Both air and rocket exhaust enter the mixing duct where the two are mixed and/or fuel is added for combustion depending on the mode of operation and the RBCC configuration. The products of combustion leave the RBCC nozzle producing thrust. The choking is achieved by employing either a physical or a thermal throat. The thermal throat is a preferred means for flow control due to the idea of employing scramjet mode of operation, which doesn't need 
a physical throat as the flow is supersonic along the entire length of the engine. As was mentioned previously, the RBCC engine usually employs different modes of operation depending on the flight conditions. These modes include ejector, ramjet, scramjet, and rocket modes of operations, shown in Figure 1.3. The next few paragraphs describe each of these modes of operation in more detail.

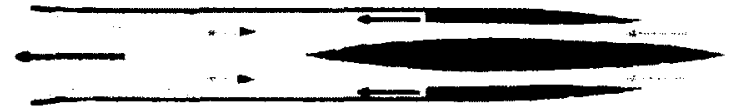

(a) Ejector Mode

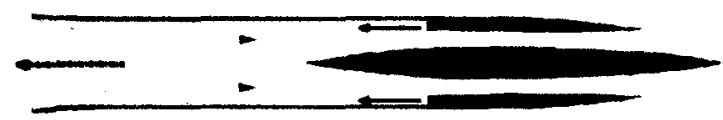

(c) Rocket Mode

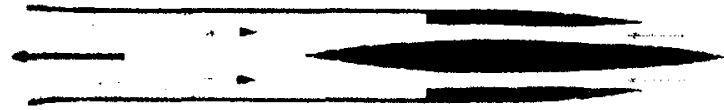

(b) Ramjet/Scramjet Mode Entrained Air Rocket Exhaust Injected Fuel RBCC Exhaust

Figure 1.3: Modes of operation of an RBCC engine.

The ejector mode of operation is used at lift-off and during the subsonic and part of the supersonic flight profile. As was shown in Figure 1.1 the ejector mode has higher specific impulse as compared to a pure rocket engine, hence. the desire to use this mode. The ejector mode is one of the few alternative to a pure rocket engine at subsonic flights. The other being turbofan/turbojet engine. The main advantage of the ejector mode over the turbojet engines is the absence of any additional heavy turbine components and a simplified flow passage. This, however, comes at the cost of specific impulse and therefore might result in the need to carry more fuel on board, though not as much as in the case of pure rocket. In the ejector mode the high energy stream that comes from the rocket engine is used to entrain air from the atmosphere through the exchange inlet. The air is then mixed with the high energy stream, which could be fuel rich (simultaneous mixing and combustion - SMC) or the fuel could be added after the mixing of the two streams - diffusion and after-burning (DAB). The unburned fuel then reacts with the oxygen in the entrained air increasing the thermal energy of the mixture, which is accelerated though the nozzle, producing thrust. For 
higher efficiency of the ejector mode a good mixing between the rocket and air streams is required. A longer mixing duct allows for better mixing, however, it also means a heavier engine, so a compromise between the two is required. There are different ways to improve the mixing of the two streams. One is the pulsing rocket stream [11]. Another way is to increase the area of interaction between the streams, as is done by multiple rocket engines. Both of these methods were extensively analysed by different authors, who came to conclusion that it is possible to reduce the length of the mixing duct to a length over diameter ratio (L/D) of 2.5 [12]. The simplest design with the rocket being in the center showed a good mixing at L/D of around 8 to 10 [13]. This means that with proper configuration of the rocket streams, it is possible to considerably reduce the length of the mixing duct. In Figure 1.2 and 1.3 the fuel is shown to be injected inside the mixing chamber. This fuel injection is not required for the case of SMC. In the case of DAB, some authors suggest a premixing of the fuel and air even before the air enters the mixing chamber. Even though there are many studies on the topic of rocket ejectors, their application is relatively scarce. This cannot be said about the next mode of operation which takes over the ejector mode at higher speeds - ramjet.

At speeds above Mach 2 but below Mach 5-7 the ramjet mode of operation can be used to propel the RBCC engine (Figure 1.3b). Although it was shown that ramjets could be used even at low subsonic speeds they were found to be inefficient until supersonic speeds [9] and they cannot operate at standstill, hence the need for an initial acceleration mode, which in the case of an RBCC engine is the rocket ejector. A ramjet is probably the simplest airbreathing engine known. It can be as simple as a pipe with a nozzle at the end, plus a fuel injection system. For a more efficient ramjet engine a bit more elaborated design is needed. The ramjet intake is designed to slow down the oncoming air and convert the dynamic pressure to static pressure (ram effect) for more efficient combustion. At low speeds dynamic pressure is low and 
as such the rise in the static pressure is also low which explains the ramjet inefficiency at low speeds. Once air is compressed the fuel is added and the thrust is generated. In this mode the high energy rocket stream is not required (though can be used), which simplifies the analysis of the engine. For supersonic flights the compression happens through the series of shocks generated by the intake structure, which slows the air to subsonic speeds. These shocks need to be taken into account during the design stage. Unfortunately, at higher Mach numbers (above Mach 5-7) the deceleration of the air to subsonic would result in static temperatures in excess of the $2000 \mathrm{~K}$, which is above the limit of many known materials, and the combustion at these temperatures would be limited due to dissociation of the molecules [14]. As such, the airflow is decelerated to lower supersonic speeds. Since the airflow is supersonic the combustion happens at supersonic or mixed supersonic/subsonic speeds, meaning the mode of operation is scramjet (which means supersonic combustion ramjet). This leads to additional challenges, which will not be described here. For more information on scramjets the reader is directed to [15].

The concept of ramjet engine came to light in early 1900 s, with further testing and development in the next years in many parts of Europe (France, Germany, Hungary, Russia and UK) and US [9]. Since then ramjets have been used to power jets (Griffin II) and many missiles (V-1, SA-4, VEGA, X-7) as early as the 1930s. Since ramjets are unable to work from a standstill they have to be initially propelled forward by other means. In the case of the RBCC engines the ejector mode of operation is used. In the case of the Griffin II the aircraft is propelled by a turbojet engine, while in the case of the missiles usually a solid rocket motor is used to initially propel them to the supersonic speed. In the 1960s the work was done on the inter continental ballistic missile to use ramjet technology - Gnom [16]. The missile was considerably lighter than equivalent solid-fuel or liquid-fuel rocket. However, the rocket was never completed due to the death of the chief designer. After a few ground tests the project 
was cancelled. As seen from the above examples the ramjet technology is quiet mature with an extensive use in military applications.

The scramjet technology on the other hand is still in development. Currently, there is extensive research being performed in the area of supersonic combustion and scramjet vehicles, with major research being done in Europe and Russia, Japan, and USA. However in all of these research works, the engines are accelerated to hypersonic speeds by means of solid rockets. To date research in scramjet propulsion has led to the creation of the X-43A achieving Mach 9.8 powered by scramjet engine [17].

The final mode of operation of the $\mathrm{RBCC}$ engine is the rocket mode. In the case of non-combined cycles, the rocket engines operate at either over-expanded (the nozzle pressure is slightly lower than atmospheric pressure) or under-expanded (the nozzle pressure is slightly higher than atmospheric pressure) nozzle exit conditions. Neither of the conditions represent the maximum nozale efficiency of the rocket. At higher altitudes the atmospheric pressure is much lower than that at lower altitude, meaning that if the rocket nozzle is designed to operate in the lower atmosphere it is going to be under-expanded in the higher atmosphere. Under-expansion means that it is still possible to accelerate the flow further, which is to improve the performance of the nozzle. In the case of the rockets one reason for multi-staging is to bring the nozzle conditions closer to the local atmospheric. In the case of the RBCC engine, the rocket mode is activated in the higher atmosphere where pressure is negligible. This means that the rocket exhaust, after leaving the rocket nozzle (Figure 1.3c) continues to expand, achieving higher speeds at the RBCC nozzle exit, which means higher performance of the engine.

From a theoretical perspective the RBCC engine has a higher overall specific impulse and therefore better performance than an equivalent rocket engine, including the performance outside of the atmosphere. From the practical perspective the RBCC engine adds more complexity over a rocket engine. This complexity comes from the 
need to combine different modes of operation and have seamless transition between these modes. Another issue is the ejector configuration, or more precisely the configuration of the rocket nozzles. As was mentioned, a simple RBCC configuration with a single centred rocket nozzle results in long and heavy mixing duct. Reduction in the mixing duct length requires a more elaborate configuration. A large number of rocket engines within the RBCC engine would result in complicated flow paths and plumbing, requiring multiple combustion chambers. One possible way to simplify this and reduce the number of parts is to have a single combustion chamber with the exhaust being diverted through an elaborate nozzle design into a circular rocket exhaust profile as proposed by Cerantola and Etele [18], [19]. This idea is the basis of the research described in this work.

\subsection{Problem Statement}

At Carleton University one possible RBCC engine design is being studied. Work on this design has led to the development of the exchange inlet which contains a modified flow path for the rocket exhaust, while allowing a smooth flow of air into the mixing duct, where air and high energy exhaust from the rocket are mixed for further combustion. The performance of the exchange inlet at subsonic speeds has been examined in previous works [20] and [21]. Although the design method was able to estimate the total pressure losses for the rocket flow path [19] as well as through the air passages due to viscous effects [20], there were no means of accounting for losses due to shocks at supersonic flight conditions.

At supersonic speeds the shock waves generated by the inlet geometry are causing the compression of incoming air and are essential to the performance of the inlet. This leads to the need to calculate the total pressure losses across the developed shocks. The work described within this text presents a method to estimate the pressure losses 
through the intake due to shocks at supersonic speeds. This method is based on shock shape fit and can be used for both sharp and blunt bodies. It provides fast estimations of the pressure losses for geometries of the type present in the exchange inlet without the need for time consuming meshing and 3D simulations. This method could be used in a genetic algorithm already developed [22] to help optimise the exchange inlet geometry over a selected flight regime. The estimations obtained for one possible geometry of the exchange inlet are presented and compared to the results from 3D numerical simulations.

\subsection{Computational Methods}

The calculations of the total pressure loss for the RBCC engine EI comes down to calculating or estinating the flow field around and within the EI to find the geometry and the strength of the generated shocks. There are a few ways of solving for the flow at supersonic speeds. One of these methods includes the solution of the full Navier-Stokes equations using Finite Volume Method (FVM), though in the case of an inviscid flow assumption, they are simplified to Euler equations. Another method is the method of characteristics, which simplifies the governing equations even further along characteristic lines. These methods are numerical approximations of the flow field and have been shown to generate reasonable results for many geometries and in many situations. These methods have their advantages and disadvantages. A different approach is to estimate the shock geometry based on an analytical or an empirical solution and use this geometry to solve for property changes across the shock. 


\subsubsection{Navier-Stokes Solver}

One of the most widely used numerical methods in fluid dynamics is Finite Volume Method for domain discretization and the solution of the governing equations. For this method the domain of interest is broken down into small elements (mesh) for which a discretized form of governing equations is solved. The smaller the elements the more closely the results model the real flow. However. reduction in the size of the elements leads to an increase in their numbers and therefore increase in the number of equations that need to be solved. This leads to an increase in computational time. This method is very versatile and can be used for either subsonic or supersonic flows, as well as for a mixed flows. However, this method has its weaknesses. In the case of supersonic flow with a shock present, the discontinuities, which are the shocks, are not necessary modelled as discontinuities. The computations for this method are done for a finite volume, and if the discontinuity happens to be inside this volume, this method will only have information on either end of the volume, meaning the discontinuity is not captured very well. The method is still able to capture the property changes across

the shock relatively well, provided the element size is small enough. Unfortunately, the optimum size of the elements is specific to each case, leading to the requirement of results validation based on different element sizes. This could result in relatively big meshes and therefore long computational times.

In addition to long computational times, the mesh generation also takes a considerable amount of time. There are mainly two types of meshes: structured or unstructured. Structured mesh means that all elements are ordered and the computation is performed in a pattern resulting in improved computational times as opposed to unstructured mesh. The elements in unstructured mesh can be numbered in any order independently of their respective position, which leads to the requirements of storing the information about which elements connects to which elements. 
This connectivity information requires additional memory for storage and requires addition computation time for the search through this information. In general the structured mesh is harder to create and more time intensive on the human side, but offers a better quality of mesh. In this work a software called ICEM CFD is being used [23]. This software allows for a structured mesh to be created and imported into the solver, which in this case is Ansys-CFX [24]. Once the structured mesh is created ICEM CFD allows for easy manipulations of the elements size, which are relatively fast to change. Alternatively, it is possible to create an unstructured mesh, which takes relatively little human time to setup, but takes a considerable amount of time to compute. Depending on the mesh size it can take from a few minutes to a number of hours to generate the mesh, making the mesh refinement process a bit more time consuming overall as compared to the structured mesh. The time required to create the mesh in certain cases can be as long as the simulation time itself. This leads many to look into mesh-less methods, which unfortunately are not available in the commercial simulation tools used for this work. Of note is that the mesh-less method computes the location of data points based on a "natural coordinate" system of the local flow [25] but does not refuire a mesh to be created prior the computations. An example of a mesh-less method is the method of characteristic described below.

\subsubsection{Method of Characteristics}

The method of characteristics is often used for fast computations of supersonic fluws around relatively simple geometries. This method is based on the constant characteristic lines along which the governing equations are transformed. In the case of supersonic flow the characteristic lines are the lines along which the partial differential equations become ordinary differential equations which are easy to solve. This method is based on the fact that the supersonic flow is hyperbolic, meaning that a given point has effect on some region downstream of it, but not upstream. The two 
boundaries of this confined region are the characteristic lines, that emanate from or intersect at a given point. In the case of rotational flow an additional characteristic is added, that represents a streamline. The flow field is calculated from an initial boundary and calculated in steps. This method has relatively few iterations, with the exception of rotational flow, where the streamline characteristic requires some additional iterations. This makes the method computationally inexpensive. The main disadvantage of this method is that it does not work for regions of subsonic or subsonic-supersonic flow.

\subsubsection{Semi-Analytical Method}

Semi analytical methods are based on many simplified assumptions required to generate the results. In the rase of supersonic flow, the analytical part of the analysis would be the change in properties of the flow across a shock. The geometry of the shock on the other hand can be found analytically only for two cases: a shock generated by a wedge of infinite thickness and length, and a shock generated by a cone. In the case of the cone shock the solution requires numerical integration [26], arguably making the wedge shock the only type of shock geometry for which a closed form solution exists. For the case of a detached shock no analytical solution exists, and as such the shock geometry needs to be solved through a numerical method or one could use an approximate shock profile, and calculate the flow properties based on this profile.

In the case of the shock profile there have been several attempts to simplify the analysis, especially in the days when powerful computers were not available. A few examples of these experimental curve fits were presented by Ambrosio [27], Billig [28] and Love [29]. Love used an elliptical profile for the bow section of the shock, while taking into account slightly different blunt body geometry configurations. Ambrosio and Billig on the other hand used a hyperbolic shock profile. Billig's shock profile is 
described in more detail in Chapter 3.

When computers became somewhat readily available, numerical methods became more popular. Some authors created interesting methods for dealing with detached shocks. These methods mainly involve a variation of a numerical differencing method on a transformed set of Euler equations. The transformations are performed for a mesh in the region around the stagnation point and the solution for the flow in the subsonic as well as the supersonic regions behind the shock are found, while calculating the geometry of the shock itself. These methods usually involve an iterative approach for solving the flow field and use transient simulations as according to [30] steady state simulations do not produce accurate results.

The next section describes the semi-analytical method that is used for estimating the flow around the EI, followed by a comparison of this method to the FVM CFD simulations performed using Ansys-CFX [24]. Through the text, CFD simulations refer to the simulations performed using Ansys-CFX, while the terms "estimations" and "estimation method" refer to the developed semi-analytical method described in Chapter 3. 


\section{Chapter 2}

\section{Exchange Inlet Geometry}

For an RBCC engine at low speeds, air is entrained with the help of a high energy gas coming from the rocket exhaust. The two streams are mixed inside a duct or a secondary combustion chamber, and then accelerated, thereby producing thrust. The exchange inlet (Figure 2.1) is designed to facilitate the mixing between these streams by means of enlarged contact area between the two streams. The rocket flow path

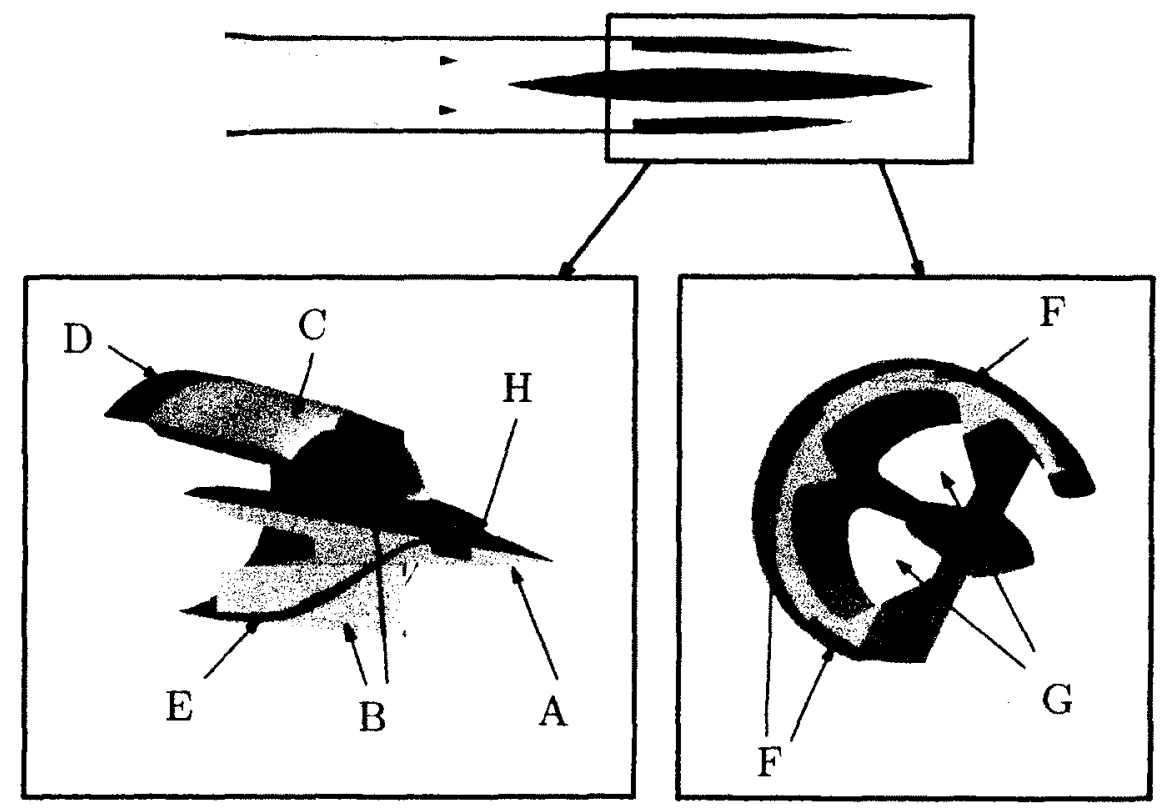

Figure 2.1: Cutaway view of EI geometry: A - Centre Body, B - Fairings, C - Cowl, D - Mixing Duct Entrance, E - Rocket Flow Path, F - Rocket Exhaust, G - Air Passage, $\mathrm{H}$ - Combustion Chamber. 
(E) is used to expand the flow of hot gases from the combustion chamber $(\mathrm{H})$ into the mixing duct (D) where it is mixed with the air entrained through the air passage (G) of the inlet. The shape of the rocket flow path is driven by the desire to increase the interaction between the air and the rocket exhaust using annular exhaust profile $(F)$, while reducing the number of pumps and the complexity associated with multiple combustion chambers. The result is a rocket flow path which diverts the rocket exhaust from a single combustion chamber into a circular stream of hot gas. The exchange inlet geometry shown in Figure 2.1 was initially analysed for subsonic flight conditions only [20], [22]. The blunt shapes of the leading edges of the cowl (C) and the fairings (B) are not well suited for supersonic flight conditions. Even though this is the case this geometry is still used to demonstrate how the high losses are estimated, since the geometry generates all of the shocks expected over the exchange inlet, including those that would be detached even for a sharper geometry. The detailed design procedures for this geometry are described by T. Waung in his thesis [20] and are not repeated here. The EI geometry used in this work is taken directly from $\mathrm{T}$. Waung results and as such is not currently optimized for any flight profile. Due to the symmetry of the geometry most of the analysis refers to the symmetry planes and the half-section of the EI all of which are shown in Figure 2.2. Symmetry Plane 1 is located between the fairings while Symmetry Plane 2 cuts the fairing into half. A single section is defined as the region between two Symmetry Plane 1, while the region bound by Symmetry Plane 1 and Symmetry Plane 2 is called half-section.

For the supersonic performance of the EI estimation method only the outer parts of the geometry are required: the centre body (A), the fairings (B), and the cowl (C). All of which are indicated in Figure 2.1. According to the design the centre body houses the combustion chamber. The rocket flow path, which diverts the high energy gas from the combustion chamber into the mixing chamber, is hidden inside the fairings and the cowl. The fairings connect the cowl to the centre body. At 


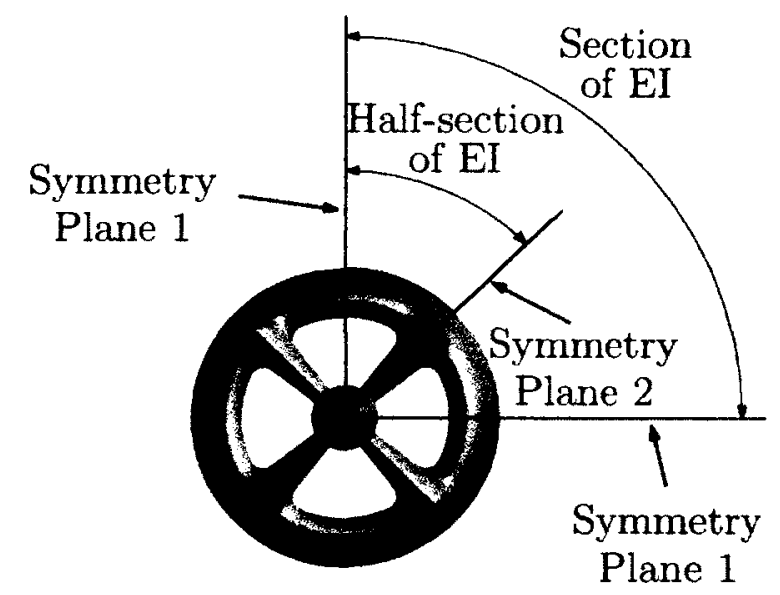

Figure 2.2: Definition of half-section of the EI and the symmetry planes.

supersonic speeds all of the outer geometries produce shocks. These shocks will be described later. First the EI geometry needs to be imported into the estimation code. Instead of using the procedures described by Wuang [20], the code reads the geometry files produced by the compiter code created by Wiang. These files contain the 2D lines for centre body and cowl geometry, which are axisymmetric, as well as the 3D surface for the fairing. The fillets between the fairings and the cowl are not imported because their effect is hard to account for. Figure 2.3 shows a half-section of the EI outer geometry imported into the estimation code and used as the base for all of the further analysis described in Chapter 3.

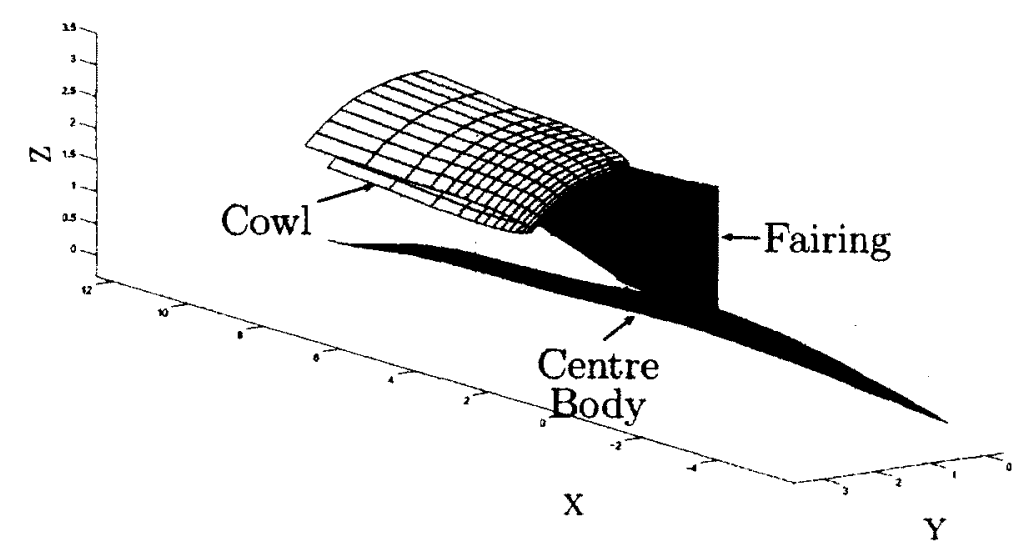

Figure 2.3: Section of EI geometry as imported into the estimation code. 


\section{Chapter 3}

\section{Estimation Method}

\subsection{Overview}

The estimation method described within this chapter is designed for fast estimations of the Exchange Inlet (EI) performance at supersonic flight conditions to select a viable geometry for further analysis. The method is not meant to provide an exact solution to the flow and is not as accurate as finite volume CFD computations, however, it is considerably faster and provides a reasonable estimate for the total pressure loss due to shocks across the EI. The estimation method is written using MATLAB software [31] and utilizes some of the MATLAB inner functions such as numerical ordinary differential equation (ODE) solver and interpolation algorithms.

The EI has three main components that generate shocks: cowl, fairings and the centre body (Figure 2.1). The shock from the centre body is approximated using a cone shock solution, while the shocks due to the other two components are approximated using a detached 2D shock solution. Figure 3.1 shows the shocks generated around the EI. As seen from the figure, the centre body shock is a cone, while the other two shocks are somewhat more elaborate.

The estimation method is built around determining the shock shapes and finding 


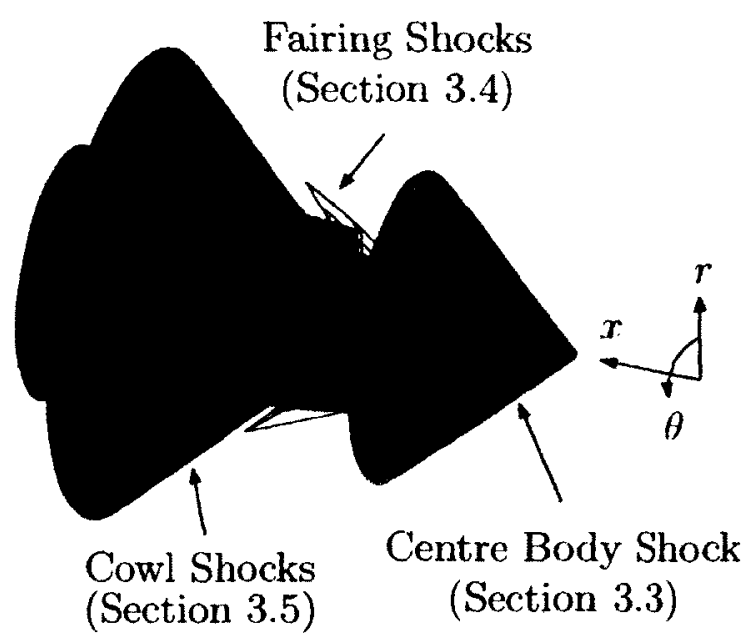

Figure 3.1: Main shocks generated by the geometry

the property changes across those shocks. The centre body generated shock is approximated using Taylor-Maccoll equation for conical shock solution [26]. The total pressure drop is obtained from the shock geometry and is the same along the entire surface of this shock. While the shock itself closely resembles a cone shock, the flow field around the centre body is affected by the curvature of the centre-body which causes the acceleration of the flow. This acceleration around the centre body is assumed to be isentropic and as such has no effect on total pressure which is the main performance criteria used in the estimation method. However, this acceleration still needs to be considered because the Mach number has effect on the next shock. The acceleration along the surface of the centre body is calculated using Prandtl-Meyer expansion function, and the flow field between the shock surface and the centre body surface is calculated from linear interpolation of the data from these two surfaces. This provides the Mach number needed to calculate the shock for the next geometry.

Once the flow field after the first shock is known the data is interpolated to find the conditions along the leading edge of the fairings. Based on these conditions the shocks are fit around the fairings (Section 3.4). For the purpose of the shock fitting, the fairing is represented as an infinite 2D blunt body. Multiple shocks are fitted 
along the leading edge of the fairing at different radii. This takes into account slight variations in the geometry of the leading edge of the fairing as well as the difference in the length of the shocks at different radii. This results in 3D shock-surface. The flow field from the previous step is projected onto this shock surface and property changes across the shock are calculated using equations described in Sections 3.2 and 3.4. The total pressure drop across this shock is averaged along the shock surface in the direction normal to the plane of the leading edge of the fairing. This results in a radial variation in total pressure, which is then projected onto the cowl shock (which assiumes isentropic flow withont any total pressure losses between the two shocks). The Mach number in this region is changing considerably due to the curvature of the fairing and the centre body. To take into account the change in Mach number certain assumptions are made to calculate the flow field between the fairing and the cowl shocks. These assumptions are described in more detail in Section 3.4.

The cowl is assumed to be an infinite 2D blunt object similar to the fairings. This allows for a relatively simple curve fit for the shock geometry. Once the shape of this shock is found, the calculated flow field from the previous shocks are interpolated to obtain the properties of the flow entering the cowl shock. This shock is then adjusted for a Mach stem, if one exists, which is calculated from the shape of the EI and the flow properties behind the cowl shock. Using this new shock shape the flow properties after the shock are adjusted to take into account the new shock geometry. After that the properties are averaged in radial direction and the total pressure drop across the $\mathrm{EI}$ is found. Section 3.5 describes this process in more details. In addition to the total pressure at the exit of the EI, the mass flow rate through the inlet is also computed (Section 3.6). 


\subsection{Isentropic compressible flow and oblique shock equations}

The following equations (Eqs. 3.1 to 3.5 ) correlate the static and stagnation conditions at a single point or along a streamline with no entropy generated along the said streamline. The derivations of these equations are available in many textbooks ( [26] and [32] are two examples) and are not included here. Eq. 3.1 correlates the total temperature $\left(T_{0}\right)$ to static temperature $(T)$ as a function of the Mach number $(M)$ and ratio of specific heats $(\gamma)$. The next equation describes the relation between total $\left(P_{o}\right)$ and static $(P)$ pressures, followed by total $\left(\rho_{0}\right)$ to static $(\rho)$ density ratio. Eq. 3.4 relates the area of a duct $(A)$ with a given Mach number at that location $(M)$ to the choking area $\left(A^{*}\right)$ for that geometry. Eq. 3.5 allows one to calculate the mass flow rate through the area $A$. provided one knows the total pressure and temperature at that location as well as the Mach number normal to that area. Eq. 3.5 is only used at the end of the analysis to calculate the mass flow rate through the EI. The rest of these equations are used throughout the analysis to find static conditions or the Mach number based on the total or stagnation conditions.

$$
\begin{gathered}
\frac{T_{o}}{T}=1+\frac{(\gamma-1)}{2} M^{2} \\
\frac{P_{o}}{P}=\left(\frac{T_{o}}{T}\right)^{\frac{\gamma}{\gamma-1}} \\
\frac{\rho_{o}}{\rho}=\left(\frac{T_{o}}{T}\right)^{\frac{1}{\gamma-1}} \\
\frac{1}{A^{*}}=\frac{1}{M}\left(\frac{1+0.5(\gamma-1) \Lambda I^{2}}{0.5(\gamma+1)}\right)^{\frac{\gamma+1}{2(\gamma-1)}}
\end{gathered}
$$




$$
\dot{m}=N A P_{o} \sqrt{\frac{\gamma}{R_{a i r} T_{o}}}\left(\frac{2}{2+(\gamma-1) M^{2}}\right)^{\frac{\gamma+1}{2(\gamma-1)}}
$$

Figure 3.2 depicts a supersonic turn around a corner by a turning angle $\theta$. The tum is considered to be isentropic. To make this turn the flow passes through a series of expansion waves (or an expansion fan) and is accelerated from $N_{1}$ to $M_{2}$. The expansion fan starts with an expansion wave at angle $\tau_{1}=\sin ^{-1}\left(1 / M_{1}\right)$ and terminates at $\tau_{2}=\sin ^{-1}\left(1 / M_{2}\right)$, both of these angles are measured with respect to the direction of the flow. $M_{2}$ is found from Prandtl-Meyer function defined by Eq. 3.6. That is $M_{2}=f\left(\nu_{2}\right)$, where $\nu_{2}=\nu_{1}+\theta$. Once $\nu_{2}$ is known $M_{2}$ is found by iterating Eq. 3.6. A detailed derivation of Eq. 3.6 is available in most textbooks on fluid dynamics ( [26], [32], [33], [34]).

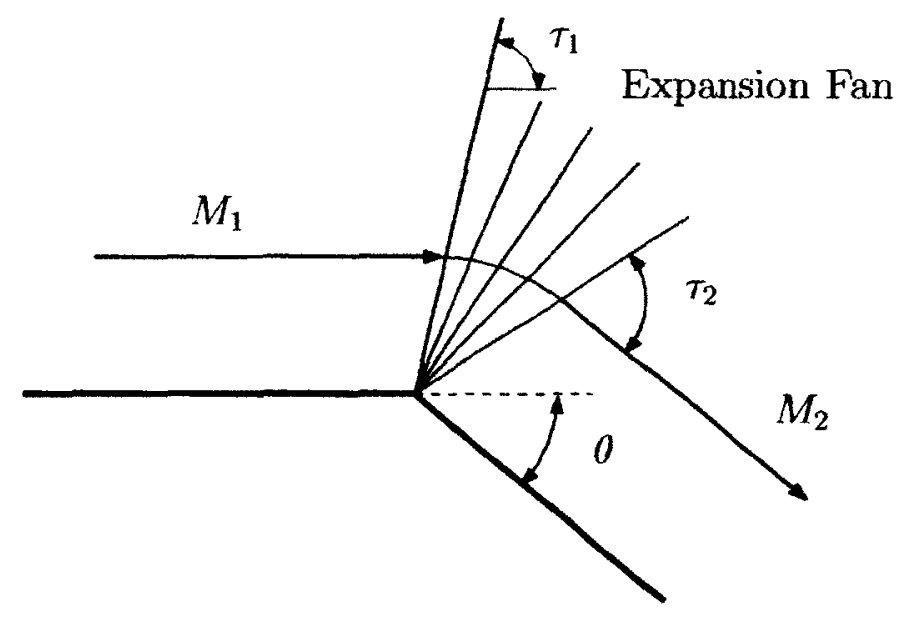

Figure 3.2: Supersonic expansion around a corner.

$$
\nu=\left(\frac{\gamma+1}{\gamma-1}\right)^{1 / 2} \tan ^{-1}\left(\frac{\gamma+1}{\gamma-1}\left(M^{2}-1\right)\right)^{1 / 2}-\tan ^{-1}\left(M I^{2}-1\right)^{1 / 2}
$$

The above equations work for an isentropic flow. Below is an example of nonisentropic flow. Figure 3.3 shows a schematic of a flow at Mach $M_{1}$ passing through an oblique shock at an angle $\beta$, resulting in the after shock Mach $M_{2}$ which is turned / deflected by an angle $\theta$ to the original flow direction. The normal components of 
the two Mach numbers are correlated through Eq. 3.7.

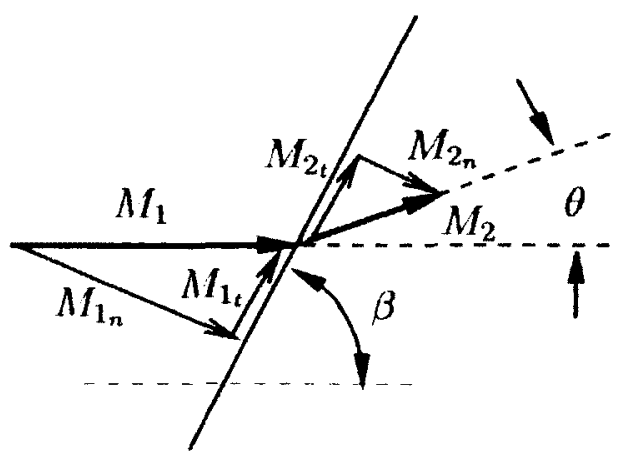

Figure 3.3: Oblique shock nomenclature.

$$
M_{2 n}^{2}=\frac{(\gamma-1) M_{1 n}^{2}+2}{2 \gamma M_{1 n}^{2}-(\gamma-1)}
$$

$M_{1 n}$ is defined as

$$
M_{1 n}=M_{1} \sin (\beta)
$$

To find the resulting Mach number after the shock the tangential component of the Mach needs to be found as well. Using the conservation of momentum in the tangential direction across the shock $\left(V_{2 t}=V_{1 t}\right)$ one can obtain the tangential component for the Mach number:

$$
\begin{gathered}
M_{2 \iota} \sqrt{\gamma K_{u i v} T_{2}}=M_{1 \imath} \sqrt{q R_{a i r} T_{1}} \\
M_{2 t}=M_{1 t} \sqrt{\frac{T_{1}}{T_{2}}}
\end{gathered}
$$


The resulting $M_{2}$ is calculated from Eq. 3.11 below:

$$
M_{2}^{2}=\Lambda_{2 t}^{2}+M_{2 n}^{2}
$$

The following equations (Eqs. 3.12 to 3.16 ) relate conditions after the shock to those before the shock.

$$
\begin{gathered}
P_{12}=\frac{P_{2}}{P_{1}}=\frac{2 \gamma M_{1 n}^{2}-(\gamma-1)}{\gamma+1} \\
\rho_{12}=\frac{\rho_{2}}{\rho_{1}}=\frac{(\gamma+1) M_{1 n}^{2}}{(\gamma-1) \Lambda I_{1 n}^{2}+2}=\frac{P_{1} M_{1 n}^{2}}{P_{2} M_{2 n}^{2}} \\
V_{12}=\frac{V_{2 n}}{V_{1 n}}=\frac{\rho_{1}}{\rho_{2}} \\
T_{12}=\frac{T_{2}}{T_{1}}=\frac{P_{2}}{P_{1}} \frac{\rho_{1}}{\rho_{2}}=\frac{P_{2}^{2} M_{2 n}^{2}}{P_{1}^{2} M_{1 n}^{2}} \\
P_{o 12}=\frac{P_{o 2}}{P_{o 1}}=\left(\frac{\rho_{2}}{\rho_{1}}\right)^{\frac{\lambda}{\gamma-1}}\left(\frac{P_{1}}{P_{2}}\right)^{\frac{1}{\gamma-1}}=\left(\frac{M_{1 n}^{2}}{M_{2 n}^{2}}\right)^{\frac{\gamma}{\gamma-1}}\left(\frac{P_{1}}{P_{2}}\right)^{\frac{\gamma+1}{\gamma-1}}
\end{gathered}
$$

The notation with two numbers in the subscript indicates the ratio between the two regions. For example $P_{12}$ in $\mathrm{Eq}_{4} 3.12$ indicates the pressure ratio for the flow going from region 1 to region 2 .

Based on the above equations it is possible to correlate the deflection of the flow $(\theta)$ with the shock angle $(\beta)$ and the upstream Mach number $\left(M_{1}\right)$ :

$$
\tan (\theta)=\frac{2 \cot (\beta)\left(M_{1 n}^{2}-1\right)}{M_{1}^{2}(\gamma+\cos (2 \beta))+2}
$$

All of the above equations are essential to calculating the properties of the flow 
around the EI geometry and provide the backbone for the analysis and the theory below.

\subsection{Centre Body Shock Estimation}

The centre body shock is estimated as a cone shock. The flow behind a shock generated by a cone can be solved using Taylor-Maccoll equation [26]. This solution takes into account 3-dimensional nature of the cone. For a cone shock there exist lines of constant properties, which emanate from the tip of the cone. These lines are shown in Figure 3.4 and 3.5. This means that $M_{2}$ is a function of $M_{1}, \beta_{c}$ (or $\theta_{c}$ ) and angle $\psi$ (shown in Figure 3.5). In fact, the solution to a shock cone is an ordinary differential equation (ODE) (Eq. 3.18), which does not have an analytical solution, as such the equation is solved numerically using ode 45 function of MATLAB [31]. Eq. 3.18 is written in non-dimensional form where $v_{r}$ and $v_{\psi}$ are components of non-dimensional velocity $\bar{V}$, shown in Figure 3.5. In this case $v_{\psi}$ is equal to the derivative of $v_{v}$. with respect to angle $\psi(\mathrm{Eq} .3 .19)$. The two components are related to the velocity term $\bar{V}$ through Eq. 3.20. The non-dimensional velocity $\bar{V}$ is related to the local Mach number $\mathrm{N}_{2}$ through Eq. 3.21. Detailed derivation of Eqs. 3.18 to 3.21 is available in [26] and [35]. A slightly different analytical approach to solving the flow field behind a cone shock is also available and is described in [36]. In this approach the ODE equation is avoided. However, numerical integration is still required, and it also requires iterations to find the solution. As a result this approach is not used in this work and will not be discussed any further.

$$
\frac{\gamma-1}{2}\left[1-v_{r}^{2}-\left(\frac{d v_{r}}{d \psi}\right)^{2}\right]\left[2 v_{r}+\frac{d v_{r}}{d \psi} \cot \psi+\frac{d^{2} v_{r}}{d \psi^{2}}\right]-\frac{d v_{r}}{d \psi}\left[v_{r} \frac{d v_{r}}{d \psi}+\frac{d v_{r}}{d \psi} \frac{d^{2} v_{r}}{d \psi^{2}}\right]=0
$$




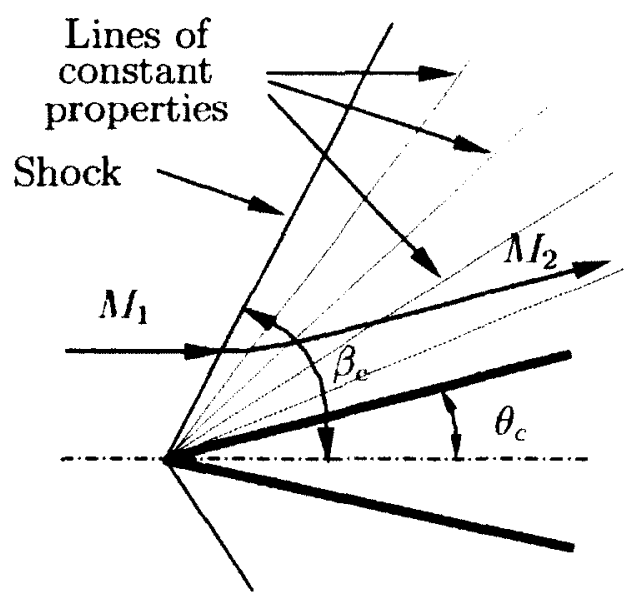

Figure 3.4: Shock generated by a cone at zero angle of attack.

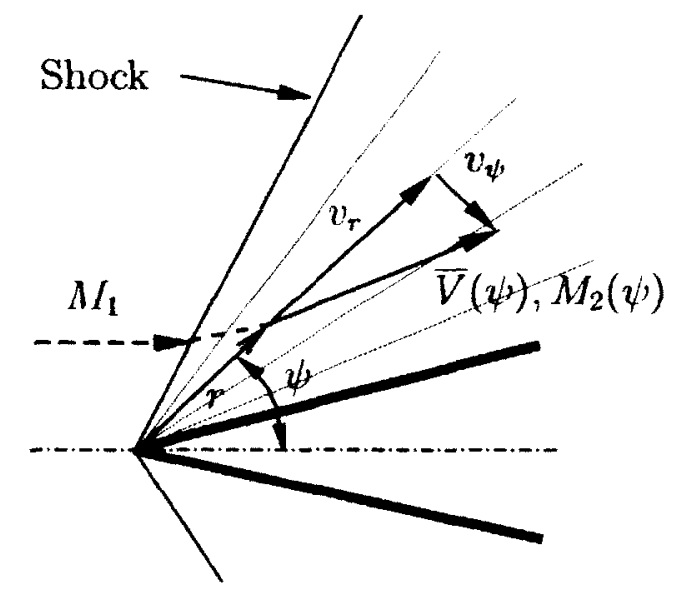

Figure 3.5: Cone shock with non-dimensional velocity components shown.

$$
\begin{gathered}
v_{\psi}=\frac{d v_{r}}{d \psi} \\
\bar{V}(\psi)^{2}=v_{r}^{2}+v_{\psi}^{2} \\
\bar{V}=\left(\frac{2}{(\gamma-1) \Lambda \Gamma_{2}^{2}}+1\right)^{-1 / 2}
\end{gathered}
$$

Eq. 3.18 is integrated from the shock $\left(\beta_{r}\right)$ to the surface of the cone $\left(\theta_{r}\right)$. The 
boundary conditions of this integration are $\frac{d v_{r}}{d \psi}\left(\psi=\theta_{c}\right)=0$ and the flow properties right after the shock which are found using Eq. 3.7 to 3.17. The fact that Eq. 3.18 is integrated starting from the shock surface means that the cone surface location or $\theta_{c}$ is not known beforehand. However, for the given problem $\theta_{c}$ is known and $\beta_{c}$ is what is needed. This leads to an iterative process of finding the desired $\beta_{c}$ for the given $\theta_{c}$. If one needs to find the cone solution for different conditions and different geometries, it might take a considerable amount of time to perform the numerical integration while continuously iterating. To speed up the calculation process the numerical integration is performed beforehand and the results for the $\beta_{r}, \theta_{r}$ and Mach number at the cone surface $M_{c}$ are stored as a function of the free stream Mach number in a database. Figure 3.6 shows the dependence of the cone shock angle $\left(\beta_{c}\right)$ on both the cone halfangle $\left(\theta_{c}\right)$ and the free stream Mach number. As seen from the figure for each half cone angle $\left(\theta_{c}\right)$ there exist two solutions: one corresponding to a weak shock (lower shock angle), and another one corresponding to strong shock (higher shock angle). In this work only the weak shock solution is of interest.

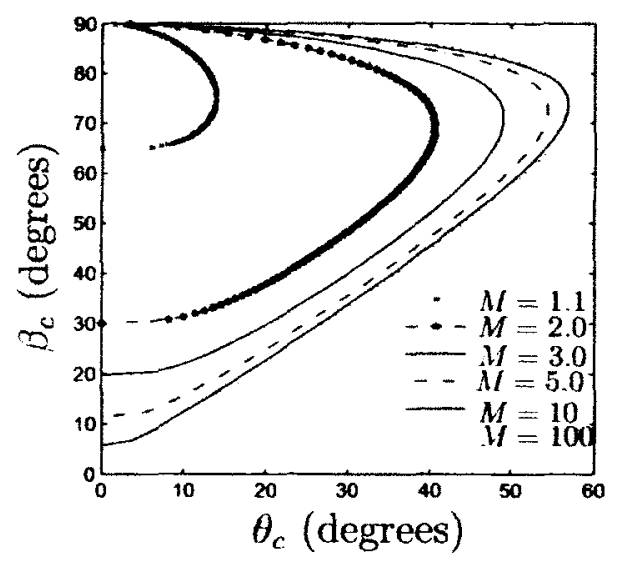

Figure 3.6: Cone shock angle dependence on the cone half-angle and the free stream Mach number.

The above describes the solution of the flow past a shock generated by a cone of infinite length. However; the surface of the centre-body is curved (as can be 
seen from Figure 2.1), this leads to acceleration of the flow along the surface of the centre body. The acceleration of the flow can be calculated by using Prandtl-Meyer expansion by starting at the tip of the centre body with $\theta=\theta_{c}$ and $M=M_{c}$, both of which are known from cone shock calculations. This yields the Mach distribution along the surface of the centre body $\left(M_{c b}\right.$ in Figure 3.7). At this point the properties of the flow are known along the surface of the centre body $\left(M_{c b}(x)\right)$ and along the downstream surface of the shock generated by this geometry $\left(M_{c s}\right)$. The flow field between the object surface and the shock requires additional computations $\left(M_{F F}\right)$. Assuming isentropic flow in this region for a given axial location both the Mach value and the direction of the flow are linearly interpolated in the radial direction between the centre body and the shock surface of the same x-location.

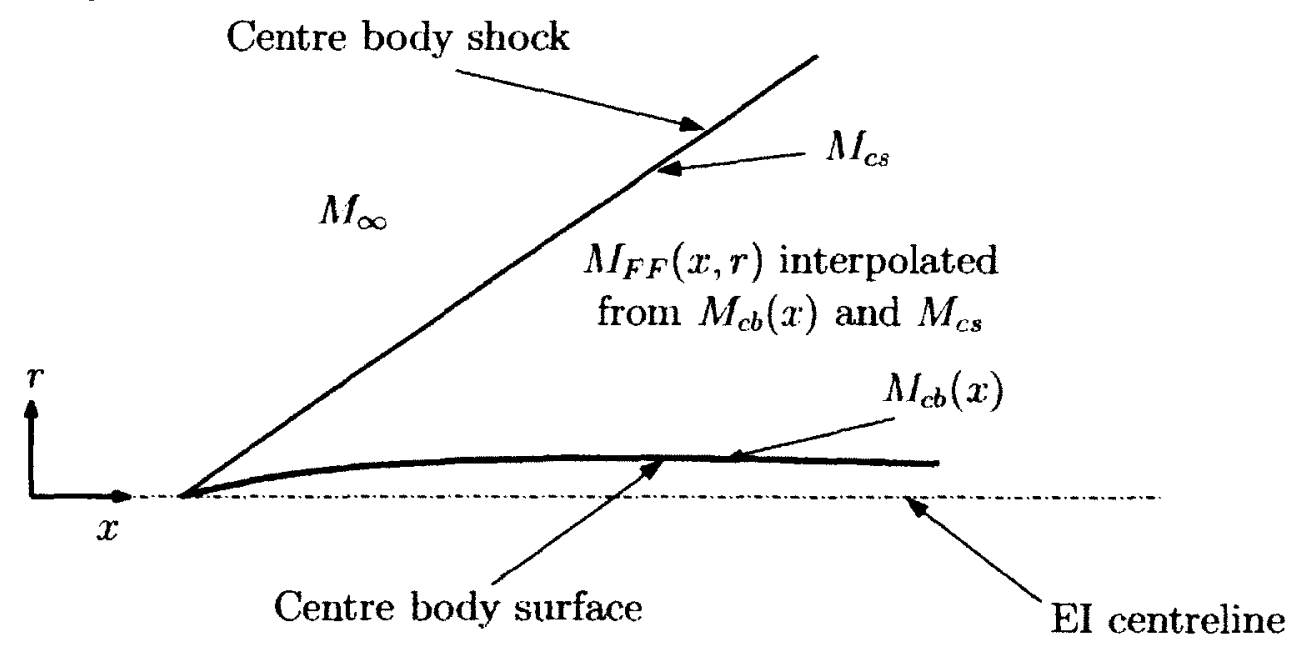

Figure 3.7: Schematic of cone shock generated by a centre body.

\subsection{Fairings Shock Estimation}

Figure 3.8 shows the EI fairings with shocks drawn around them. This is a zoomed in version of Figure 3.1 without centre body and cowl shocks. The detached bow shocks (shown) are caused by supersonic flow that comes after the centre body shock $\left(M_{F F}(x, r)\right)$. Before going into detailed analysis of the detached bow shock, a simple 
shock generated by a $2 \mathrm{D}$ wedge is considered.

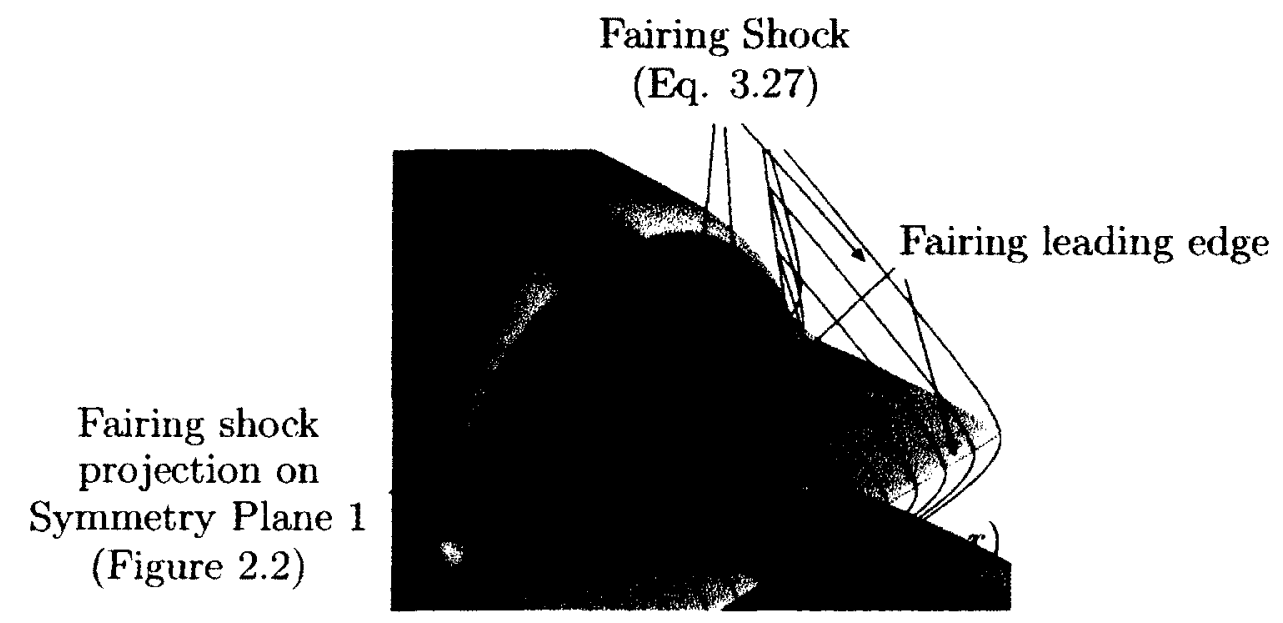

Figure 3.8: Shock generated by the fairing.

The equations described in Section 3.2 can be used to calculate the change in properties of the flow passing through a shock generated by a wedge. The schematic of such shock is shown in Figure 3.9. For the wedge the flow after the shock is aligned with the surface of the wedge. Hence Eq. 3.17 can be rewritten as Eq. 3.22 which relates the wedge angle $\theta_{w}$ and the shock angle $\beta_{w}$. To obtain $\beta_{w}$ one would have to iterate Eq. 3.17, since it is not possible to solve for $\beta_{w}$ directly.

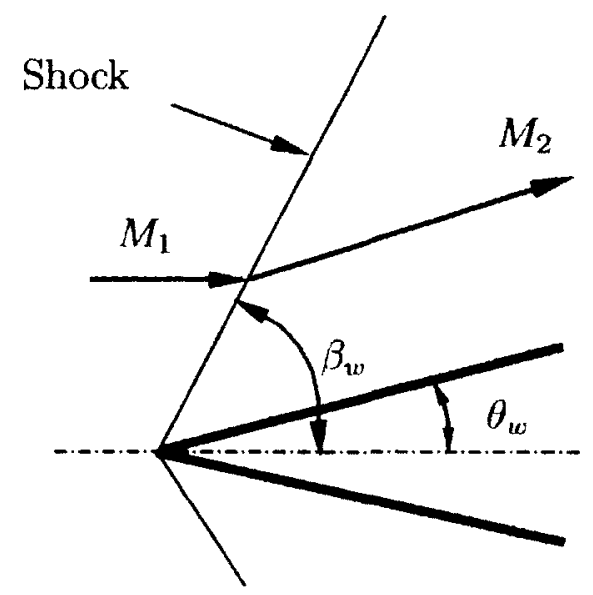

Figure 3.9: Shock generated by a wedge. 


$$
\tan \left(\theta_{u}\right)=\frac{2 \cot \left(\beta_{w}\right)\left(M_{1 n}^{2}-1\right)}{M_{1}^{2}\left(\gamma+\cos \left(2 \beta_{w}\right)\right)+2}
$$

By differentiating Eq. 3.22 with respect to $\beta_{w}$ one can obtain the shock angle at which the maximum deflection of the flow can be obtained at a given Mach number (Eq. 3.23) for which the shock is still attached. By substituting $\beta_{\theta_{d}}$ into Eq. 3.17 one can obtain the maxinum deflection angle $\left(\theta_{d}\right)$.

$$
\beta_{\theta_{d}}=\sin ^{-1}\left(\frac{\gamma+1}{4 \gamma M^{2}}\left\{M^{2}-\frac{4}{\gamma+1}+\left[M^{4}+8\left(\frac{\gamma-1}{\gamma+1}\right) M^{2}+\frac{16}{\gamma+1}\right]^{1 / 2}\right\}\right)^{1 / 2}
$$

If the wedge angle $\theta_{w}$ is larger than $\theta_{d}$ then the flow detaches from the wedge and a strong shock is formed. This situation looks similar to the one shown in Figure 3.10. An analytical solution to the detached shock is not available, however, there are other various approaches to solving for the shape of the detached shocks. Many of these involve the numerical solution of Euler's equations in one form or another. One of the examples includes a moving boundary method in which a mesh boundary is set as the shock and the location of that boundary is found through iterations, while at the same time solving for the flow field between the shock and the blunt object. One such method is described in [30]. A more general Finite Volume Method (FVM) is another example [37]. However, both of the above examples require considerable amount of computation resources to produce reasonable results, especially the FVM (used by Ansys-CFX). as they solve for the entire flow field after the shock. To keep the calculation time as short as possible a curve fit solution to the shape of the detached shock is used. Once the shape of the shock is known it is possible to find the flow properties right behind the shock using the equations described in the previous sections (Eqs. 3.7 to 3.17 ). 


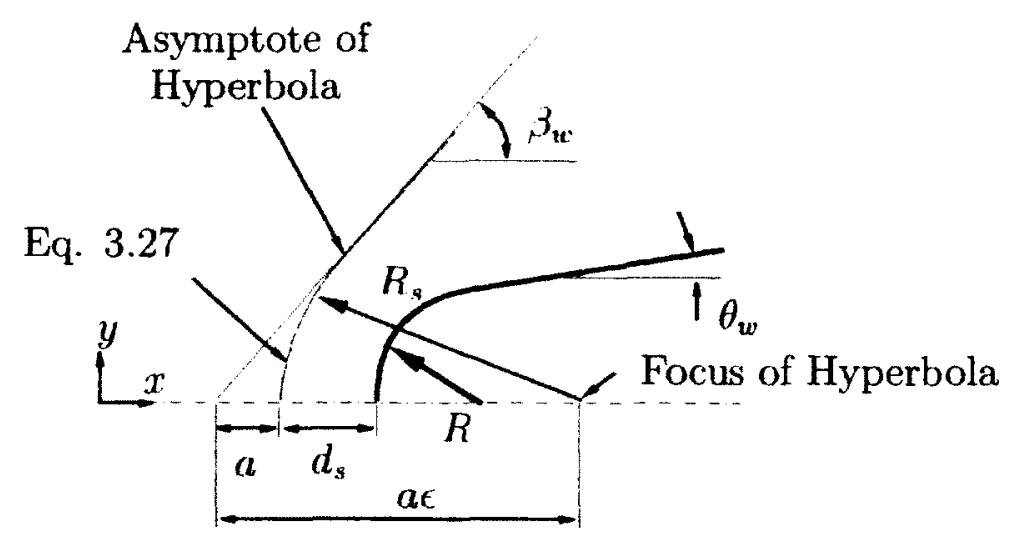

Figure 3.10: Variables used to determine detached shock geometries.

The proposed method requires the determination of the stand-off distance of the shock $d_{s}$ (shown in Figure 3.10) and the overall shape of the shock. Figure 3.10 shows the general schematic of a detached shock produced by a blunt object with several important parameters indicated: $R$ is the radius of the blunt leading edge of the object, $\theta_{w}$ is the angle at which the surface of the object leaves the blunt end (this is neasured with respect to the incoming flow direction and is a property of the fairing or cowl geometry), $d_{s}$ is the stand-off distance of the shock, $R_{s}$ is the radius of the curvature of the shock at the vertex, and $\beta_{w}$ is the angle of a $2 \mathrm{D}$ shock generated by a wedge deflection $\theta_{w}$ (Eq. 3.22). The stand-off distance is not actually required for pressure loss estimations across a single shock. However, when the intersections between the fairing and cowl shocks becomes important the stand-off distance is required to find the location of these intersections.

Though there is an analytical approximation to stand-off distance, it is applicable only at Mach numbers greater than five [34]. Instead, the shock stand-off distance equation (Eq. 3.24) taken from Billig [28] is used:

$$
\left(d_{s} / R\right)_{c y l i n d e r}=0.386 \exp \left(\frac{4.67}{\Lambda I^{2}}\right)
$$


Since $R$ is a property of the geometry (either fairing or cowl) it is known which allows $d_{s}$ to be found for a given Mach number.

With the values of $R$ and $\beta_{w}$ determined it is possible to use a hyperbolic profile of the following form [28]

$$
x=R+d_{s}-R_{s} \cot ^{2}\left(\beta_{w}\right)\left[\sqrt{\left(1+\frac{y^{2} \tan ^{2}\left(\beta_{w}\right)}{R_{s}^{2}}\right)}-1\right]
$$

to represent the shock shape (the origin is set at the center of the body curvature $R$ ). The variable $R_{s}$ is the radius of curvature of the shock, which for a cylinder according to Billig [28] is found from

$$
\left(R_{s} / R\right)_{\text {cylinder }}=1.386 \exp \left(\frac{1.8}{(\Lambda-1)^{0.75}}\right)
$$

For higher Mach numbers these equations provide good estimates for the shock shape in the immediate proximity to the blunt body. However, at distances further away from the body and at lower Mach numbers these approximations decrease in accuracy. The difference between the shock profile described by Billig (Eqs. 3.25 and 3.26) and the CFD simulations are discussed in Section 4.4 in more detail. Due to these differences a slightly modified procedure is used.

A hyperbola-like shock shape is still maintained using $\mathrm{Eq}$. 3.27 with $n$ parameter set to 1.7. $x_{o}$ is the location of the leading edge of the object. The value of $d_{s}$ is found using Eq. 3.24. Parameters $a$ and $b$ are related to the shock angle $\beta_{w}$, through $\mathrm{Eq}$. 3.28. The value of $\beta_{w}$ is found from Eq. 3.22 based on the known deflection angle $\theta_{w}$ (obtained from geometry) and the upstream Mach number. The value of $a$ is found from Eq. 3.29, where $\epsilon$ is defined by Eq. 3.30 and $R_{s}$ is estimated based on Eq. 3.31. Eq. 3.31 is based on curve-fit of the shock from 2D CFD simulations described in Chapter 4. 


$$
\begin{gathered}
\frac{\left(x-x_{o}+a+d_{s}\right)^{n}}{a^{n}}-\frac{y^{n}}{b^{n}}=1 \\
\frac{b}{a}=\operatorname{lan}\left(\beta_{\omega}\right) \\
a=\frac{R_{s}}{\epsilon-1} \\
\epsilon=\sqrt{1+\tan ^{2}\left(\beta_{w}\right)} \\
K_{s} / K=0.1589 \exp \left(\frac{6.3366}{M}\right)+1.9187
\end{gathered}
$$

This approximation provides slightly better results especially at lower Mach numbers $\left(M_{\infty}<2.0\right)$. Once the shock shape has been determined it can be applied to the leading edge of either fairing or cowl and the changes in properties across the shocks can be easily calculated using the equations for a $2 \mathrm{D}$ oblique shock.

Figure 3.11 shows a cut of the EI geometry with the example of the shock plane and the shock geometry. The calculations described above are performed for a number of shock planes located at different radii aloug the leading edge of the fairing. The exact number of shock planes is set by the user. The shock geometry is calculated based on the normal component of the Mach number along the leading edge of the fairing which is interpolated from the flow field calculated in Section 3.3. Once the shape of the shock is known the projection of the Mach number onto the shock plane $\left(M_{r y}\right)$ is again interpolated from the previously calculated flow field. The property changes across the shock are calculated from Eqs. 3.7 to 3.16 using local value of the shock angle $\beta$ along the shock line. Should the cone shock intersect the leading edge of the fairing or the fairing shock then the free stream conditions are used above 
the location of the intersection. The process is repeated for all of the shock planes resulting in a 3D surface for the fairing shock with all parameters known. At this point the total pressure behind the shock is averaged in $y$-direction.

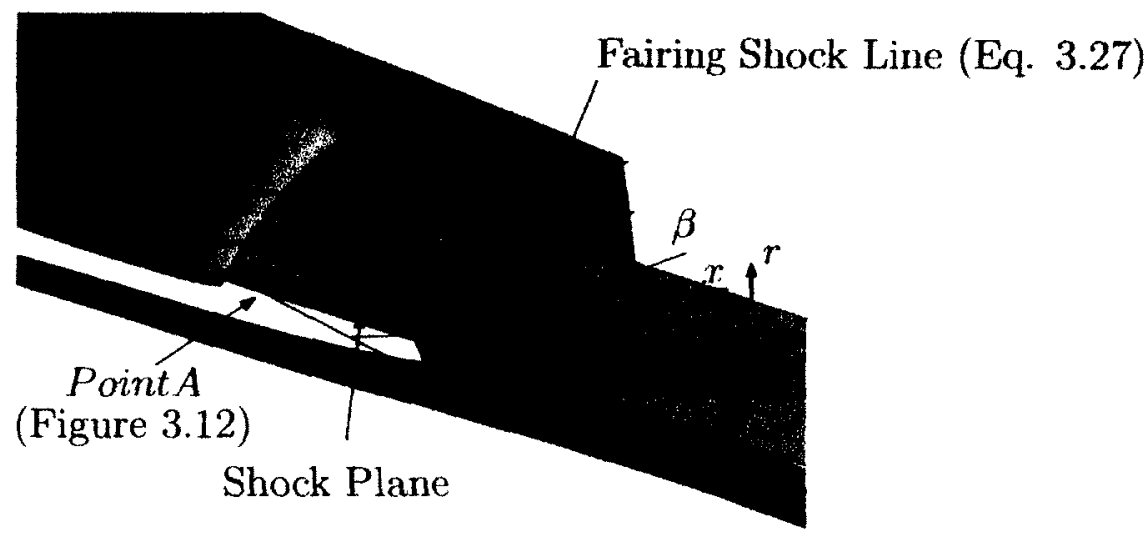

Figure 3.11: Shock generated by the fairing with shock-plane shown.

In terms of total pressure drop due to the presence of the fairings the above information is sufficient. However, due to the curvature of the fairing the flow is reacceleration which affects the cowl shock. If one assumes that the Mach wave drawn from the Point $\mathrm{A}$ in Figure 3.12 on the fairing shock back to the surface of the fairing is linear and has the same Mach number along the entire Mach wave, then this wave can be used as a starting line for the calculation of the flow field around the fairing. The angle of the slope of the surface at the point where this Mach wave intersects the object (Point B) is considered to be a reference angle. Based on the assumption that the Mach number at this point is the same as at point $\mathrm{A}$, one can calculate the Mach distribution along the surface by using the Prandtl-Meyer function. This results in an $x-y$ Mach distribution at every shock plane. To simplify the flow field the data is averaged in $\mathrm{y}$-direction. This process is repeated for all the shock planes resulting in an $\mathrm{x}-\mathrm{r}$ distribution of the Mach number. This method works only if Mach number at point $A$ is larger or equal to one. It is possible for the resultant Mach to be lower than 
Direction of the flow

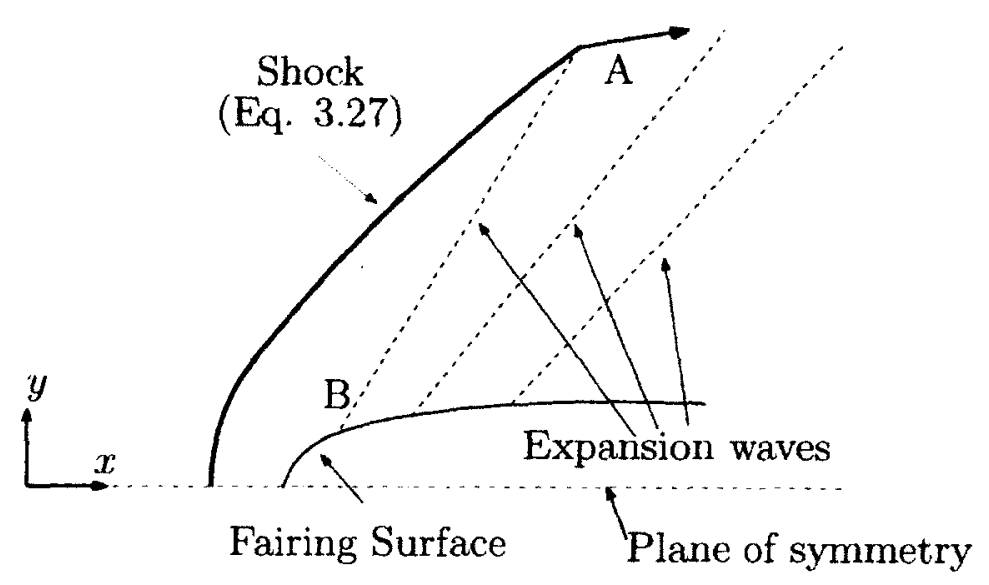

Figure 3.12: Flow acceleration around the fairing.

one (e.g. at lower $M_{\infty}$ ). In such cases the re-acceleration of the flow is not computed, this also means that the cowl shock is not computed either, since the computed flow is subsonic after the fairing shock.

In the actual EI analysis, Point $\mathrm{A}$ is always located on the symmetry plane (see Figure 3.11) away from the fairings which reflects the shock. All shock reflections are ignored for two reasons. One reason is that due to the inclination of the symmetry plane to the plane of the shock the reflected shock can no longer be treated as two dimensional, and as such becomes too complicated for the given method. The second reason is that due to the inclination of the symmetry plane the reflected shock is considerably weaker than the incident shock, and as such does not contribute significantly to the pressure drop, hence it is ignored. Same applies to the reflection of expansion waves.

\subsection{Cowl Shock Estimation}

The initial shock shape for cowl shock is estimated in a similar manner as it is done with the fairing shock, except there is a single shock line computed for the cowl as opposed to a set of shock lines computed for the fairing. Similar to the fairing, the 
cowl shock geometry is based on the flow condition interpolated from the flow field at the leading edge of the cowl. The formulation of the generated detached shock is the same as in the case of the fairing, that is Eqs. 3.24 and 3.27 to 3.31 are used. The complication for the cowl shock comes from the Mach stem, which cannot be ignored. Below is a short introduction of the reflected shock, which needs to be understood before analysing the Mach stem.

In the cases where a supersonic flow remains supersonic after passing through a shock and encounters a surface, the flow would have to make a turn by passing through another shock. Figure 3.13a shows a schematic of this situation. In this case the fluid is moving at Mach $M_{1}$ before encountering the first shock. After passing the first shock the new Mach number is $M_{2}$, which in this case is above one. The flow is now moving at angle $\theta_{r}$ with respect to the surface, which means it has to turn by that angle to be aligned with the surface again. Since at this point the flow is supersonic, to make this turn it has to pass through another shock (reflected shock). The situation where $\theta_{r}$ is lower than the maximum deflection angle $\theta_{d}$ is called a regular reflection and the flow behaves as shown in Figure 3.13a.

In cases where $\theta_{r}$ is larger than the maximum deflection angle $\theta_{d}$ the flow can no longer be tumed sufficiently using an attached shock and instead a strong shock, called a Mach stem, is generated at the surface. This situation is shown in Figure 3.13b. The flow passing through the Mach stem becones subsonic, while above the Mach stem the flow is either subsonic or supersonic and passes through a weak oblique shock (reflection shock). The resulting $\theta_{r_{s t e m}}$ is lower than $\theta_{d}$ and the flow above the slipstream is moving at an angle towards the surface.

The size of the Mach stem depends on the downstream conditions. Ben-Dor [38] describes a method to calculate the size of the Mach stem which works for a finite wedge geometry and a known back pressure. To use this method one first requires the direction of the flow after the reflected shock $\left(\theta_{s s}\right)$. A close up of the Mach reflection 


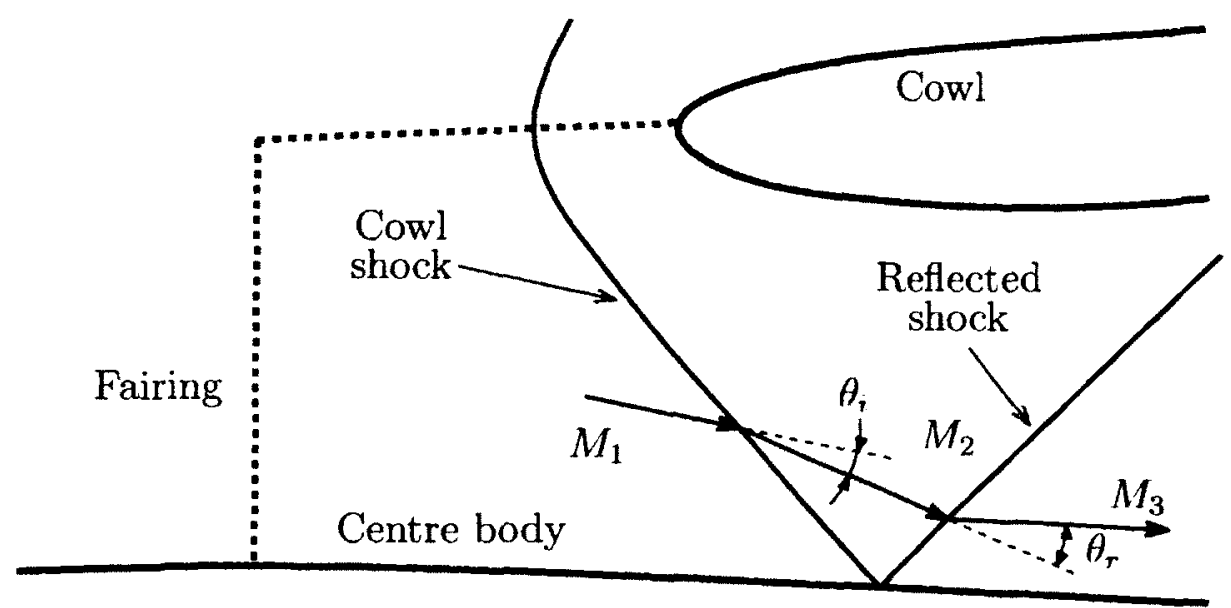

(a) Regular shock reflection $\left(\theta_{r}<\theta_{d}\right)$.

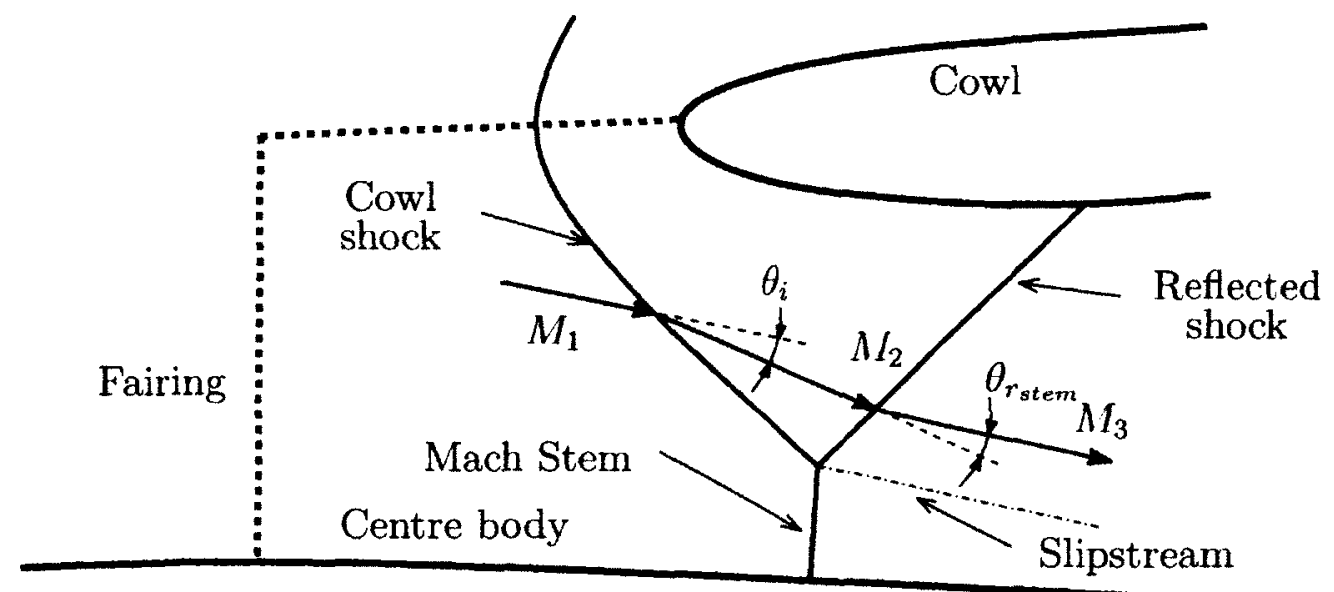

(b) Mach reflection $\left(\theta_{r}>\theta_{d}\right)$.

Figure 3.13: Possible reflection shocks.

case is redrawn in Figure 3.14 with the main variables indicated. In this figure, the Mach stem is assumed to be a normal shock. Subscript $i$ denotes the cowl (incident) shock while subscript $r$ stands for the reflected shock, and $s s$ is the slipstream. The numbered subscripts indicate the conditions upstream of the cowl shock (1); the conditions between the cowl shock and reflected shock (2); the conditions after the reflected shock (3); and the conditions under the slipstream after the Mach stem (4). The direction of the slipstream $\left(\theta_{s s}\right)$ can be found by noting that the static pressure below the slipstream and above it at the triple point are equal thus $P_{3}=P_{4}$. 


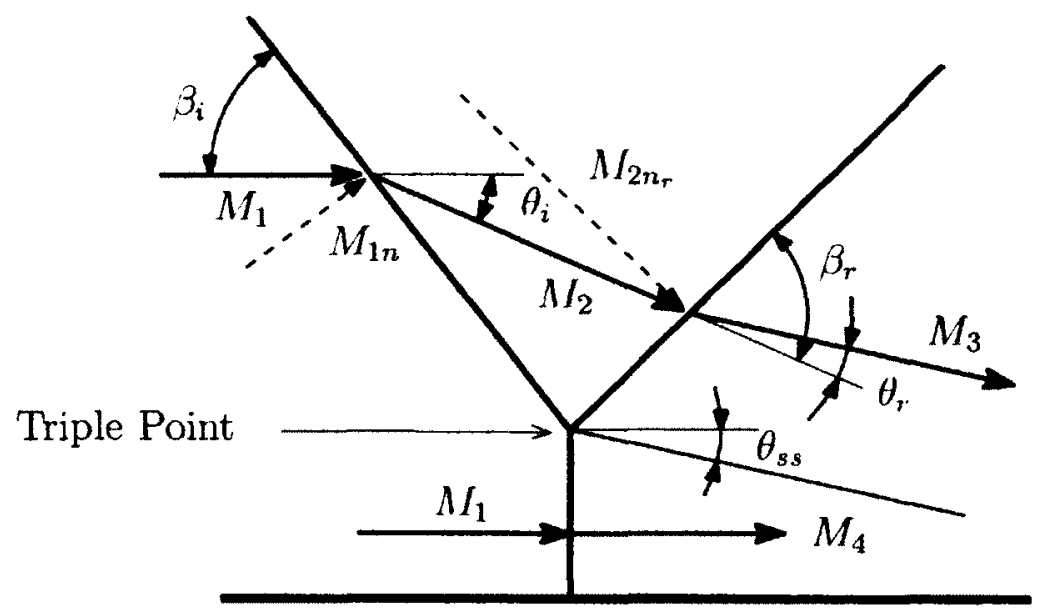

Figure 3.14: Variables required for Mach reflection analysis.

The conditions below the slipstream can be found by applying Eq. 3.12 (rewritten below)

$$
P_{14}=\frac{P_{4}}{P_{1}}=\frac{2 \gamma M_{1}^{2}-(\gamma-1)}{\gamma+1}
$$

where the pressure behind the Mach stem can be found using

$$
P_{4}=P_{1} P_{14}
$$

Similarly

$$
P_{2}=P_{1} P_{12}
$$

and

$$
P_{3}=P_{2} P_{23}=P_{1} P_{12} P_{23}
$$

The only unknown in Eq. 3.35 is $P_{23}$, which also requires $M_{2}$. Combining the above equations and the fact that $P_{3}=P_{4}, P_{23}$ can be found: 


$$
P_{23}=\frac{P_{14}}{P_{12}}=\frac{2 \gamma M_{1}^{2}-(\gamma-1)}{2 \gamma M_{1 n}^{2}-(\gamma-1)}
$$

where $M_{1 n}=M_{1} \sin \left(\beta_{i}\right)$, which is found from the fitted solution for the cowl shock. On the other hand $P_{23}$ is defined as

$$
P_{23}=\frac{2 \gamma M_{2 n_{r}}^{2}-(\gamma-1)}{\gamma+1}
$$

where $M_{2 n_{r}}=M_{2} \sin \left(\beta_{r}\right)$. The subscript $r$ in this case indicates that the normal component is with respect to the reflected shock. By combining the above two equations one can find $M_{2 n_{r}}$ :

$$
\begin{gathered}
M_{2 n_{r}}=\left(\left[\frac{2 \gamma M_{1}^{2}-(\gamma-1)}{2 \gamma M_{1 n}^{2}-(\gamma-1)}(\gamma+1)+(\gamma-1)\right] \frac{1}{2 \gamma}\right)^{1 / 2} \\
\beta_{r}=\sin ^{-1}\left(M_{2 n_{r}} / M_{2}\right)
\end{gathered}
$$

While $M_{2}$ can be found by following procedures for an oblique shock as outlined in Section 3.2 (Eqs. 3.7, 3.10 to 3.12 and 3.15).

With $M_{2}$ and $M_{2 n_{r}}$ known, $\theta_{r}$. can be found using Eq. 3.17 :

$$
\operatorname{lan}\left(\theta_{r}\right)=\frac{2 \cot \left(\beta_{r}\right) M_{2 n_{r}}^{2}-1}{M_{2}^{2}\left(\gamma+\cos \left(2 \beta_{r}\right)\right)+2}
$$

The angle of the slipstream is then the difference between the $\theta_{i}$ and $\theta_{r}$ (Eq. 3.41), while the conditions at 3 are completely defined once the conditions at 2 have been found (Figure 3.14).

$$
\theta_{s s}=\theta_{i}-\theta_{r}
$$

If one assumes that the flow passing through the Mach stem is going to choke 
further downstream, then knowing the conditions directly behind the Mach stem $\left(M_{4}\right)$ and assuming isentropic flow one can find the ratio of the area of the Mach stem to the throat area through Eq. 3.4. Therefore, assuming the slipstream doesn't change its direction the size of the Mach stem can be found if the throat area and its locations are known $\left(A^{*}\right.$ and $x^{*}$ ). Figure 3.15 shows a case where the cowl shock is generated by a sharp wedge. In this case an expansion wave emanates from the end of the wedge causing the flow above the slipstream $\left(M_{3}\right)$ to align itself with the lower surface, and hence with the direction of the flow passing through the throat $\left(M^{*}\right)$, which must be parallel to the the surface. This approach follows the ones discussed in more detail by Ben-Dor [38] and Moutan [39].

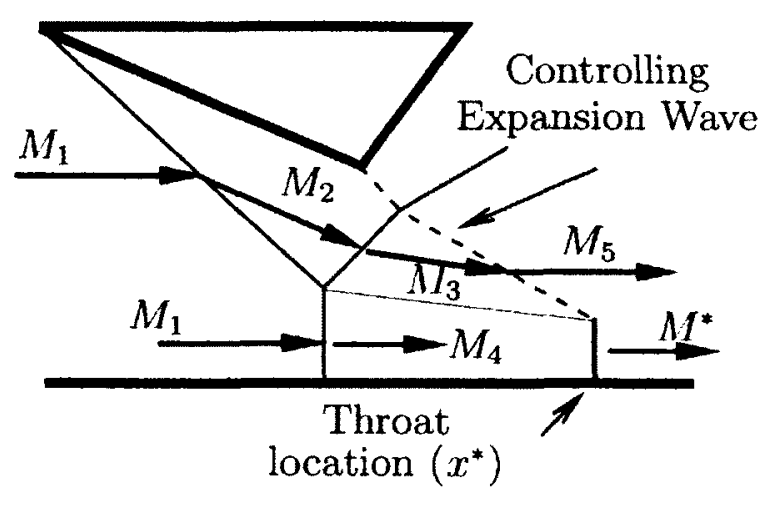

Figure 3.15: Mach reflection.

For the current geometry of the EI and more specifically the cowl region (as this is the region where the Mach stem may develop) one notes that the shock generated by the geometry is not a simple oblique shock, nor is the shape of the cowl a sharp wedge. Figure 3.16 shows the schematic of the shock geometry generated by the cowl. The figure also shows the fact that there is an angle between the surface of the centre body and EI axis $\left(\theta_{c b}\right)$ which needs to be considered. Unlike the case with a sharp wedge, the expansion wave doesn't have a single location of origin. Instead, 
the expansion wave is generated as the flow gradually turns around the surface of the cowl. Despite this, only a simple expansion wave is considered, which emanates from the cowl at a location determined as follows. Figure 3.16 shows the case where the expansion wave intersects the reflected shock. In this case, after the intersection point the expansion wave corresponds to $M_{5}$, which is parallel to the surface of the centre body. The slope of the expansion wave is equal to the slope of the Mach wave generated by the flow at $M_{5}$.

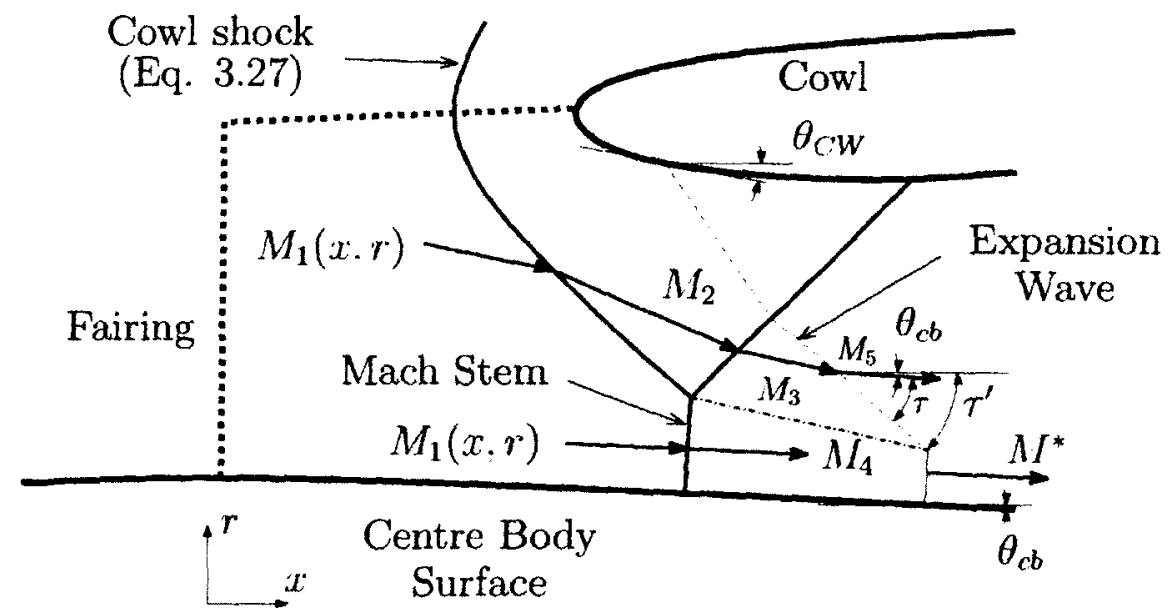

Figure 3.16: Side view of cowl shock structure.

The value of $M_{5}$ and therefore the slope of the expansion wave comes from the following analysis. Assuming isentropic flow between $M_{4}$ and $M^{*}$, and between $M_{3}$ and $M_{5}$, one can write $P_{u 4}=P_{0}^{*}$ and $P_{w 3}=P_{w 5}$, and using the definition of the stagnation pressure this yields

$$
\begin{aligned}
& P^{*}=\left(\frac{\gamma+1}{2+(\gamma-1) M_{4}^{2}}\right)^{\frac{\gamma}{\gamma-1}} P_{4} \\
& P_{5}=\left(\frac{2+(\gamma-1) M_{5}^{2}}{2+(\gamma-1) M_{3}^{2}}\right)^{\frac{\gamma}{\gamma-1}} P_{3}
\end{aligned}
$$

Equating Eqs. 3.42 and 3.43 (since the static pressures above and below the slipstream are equal) one can write Eq. 3.44 for $M_{5}$, where $M_{3}$ and $M_{4}$ are known 
from the previous shock and Mach stem analyses. The angle of the slope of the Mach wave at 5 is then $\tau=\sin ^{-1}\left(1 / M_{5}\right)$ (with respect to the direction of the $M_{5}$ ).

$$
M_{5}=\left[\left(\frac{2+(\gamma-1) M_{3}^{2}}{2+(\gamma-1) M_{4}^{2}}(\gamma+1)-2\right) \frac{1}{\gamma-1}\right]^{1 / 2}
$$

Although this yields the slope of the expansion wave hetween the reflected shock and the throat, this angle changes through the reflected shock. To calculate this change Figure 3.17 shows the schematic of a shock/expansion wave interaction, with a slipstrean generated from the point of intersection. The flow above the slip stream comes from a common region $A$, passes first through the expansion wave into region $B$, and then through an adjusted shock into region $C$. The flow below the slipstream comes from a common region $\mathrm{A}$, passes through the shock wave into region $\mathrm{D}$, and then through an adjusted expansion wave into region $\mathrm{E}$. The $\tau$ angles are the angles of the expansion waves and correspond to the Mach waves, that is $\tau_{B}=\sin ^{-1}\left(1 / M_{B}\right)$ and $\tau_{E}=\sin ^{-1}\left(1 / M_{E}\right)$. The prime sign, indicated in Figure 3.17, means that the angle is measured with respect to the horizon. The switch between the horizon reference angles and the angles measured with respect to some local direction is required for simplification of the analysis.

There are two conditions that apply along the slipstream shown in Figure 3.17: pressure is the same on both sides of the slipstream $\left(P_{C}=P_{E}\right)$, and the direction of the flow is the same $\left(\theta_{C}^{\prime}=\theta_{F}^{\prime}\right)$. For $P_{C}$ and $P_{F}$ to be equal, $P_{A C}$ should be equal to $P_{A E}$ which can be found from isentropic flow and shock equations:

$$
\begin{aligned}
& P_{A C}=P_{A B} P_{B C}=\left(\frac{2+(\gamma-1) M_{A}^{2}}{2+(\gamma-1) M_{B}^{2}}\right)^{\frac{\gamma}{\gamma-1}}\left(\frac{2 \gamma M I_{B n}^{2}-(\gamma-1)}{\gamma-1}\right) \\
& P_{A E}=P_{A D} P_{D E}=\left(\frac{2 \gamma M_{A n}^{2}-(\gamma-1)}{\gamma-1}\right)\left(\frac{2+(\gamma-1) M_{D}^{2}}{2+(\gamma-1) M_{E}^{2}}\right)^{\frac{\gamma}{\gamma-1}}
\end{aligned}
$$


Expansion

Wave

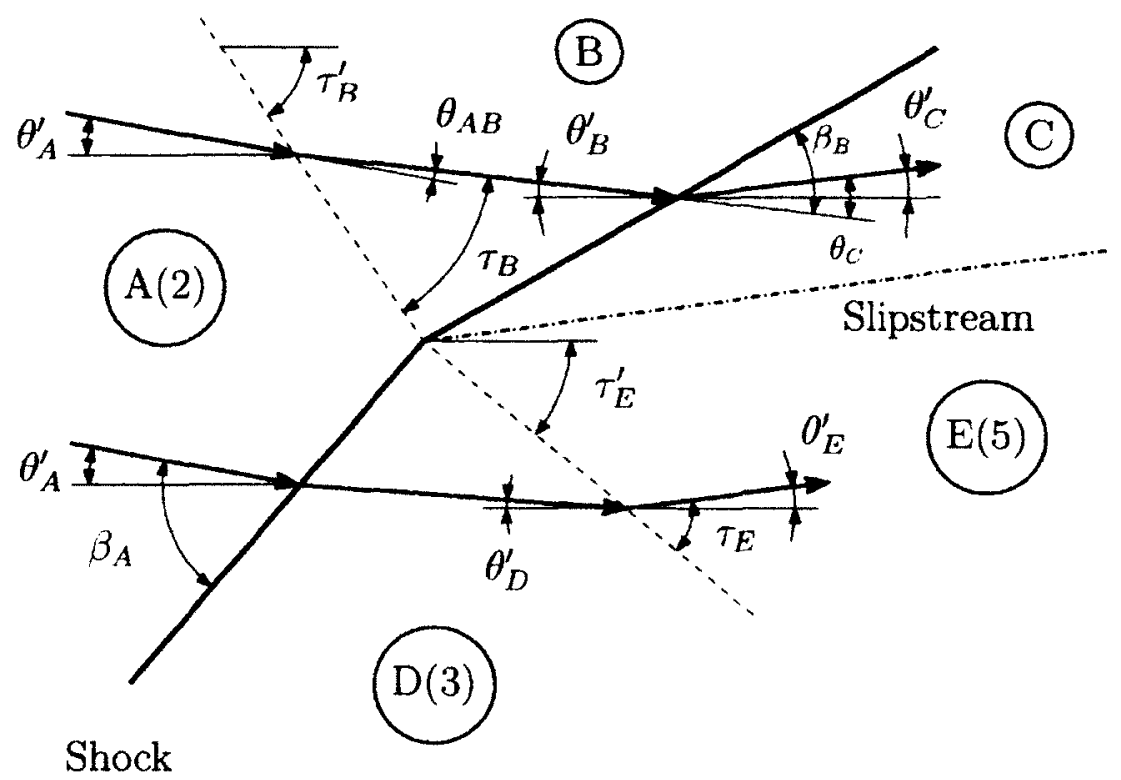

Figure 3.17: Shock/Expansion wave intersection (numbers in brackets indicate the regions shown in Figure 3.16 from previous analysis).

For the EI geometry all of the required information for $P_{A E}$ is known from the previous analysis (Figure 3.16, $M_{A n}=M_{2 n_{r}}, M_{D}=M_{3}$ and $M_{E}=M_{5}$ ) and thus the only unknowns in the above set of equations are $M_{B}$ and $M_{B n}$ which are related through $M_{B n}=M_{B} \sin \left(\beta_{B}\right)$. Applying Eq. 3.17 to the shocks between regions B and $\mathrm{C}$ yields

$$
\tan \left(\theta_{C}\right)=\frac{2 \cot \left(\beta_{B}\right)\left(M M_{B n}^{2}-1\right)}{M_{B}^{2}\left(\gamma+\cos \left(2 \beta_{B}\right)\right)+2}
$$

where $\theta_{C}$ is the turning angle for the flow passing from region $\mathrm{B}$ to $\mathrm{C}$.

$\theta_{C}$ is related to $\theta_{C}^{\prime}$ through $\theta_{C}^{\prime}+\theta_{B}^{\prime} . \theta_{C}^{\prime}$ is the direction of the slipstream and is equal to $\theta_{E}^{\prime}$, which in turn is the direction of $M_{5}$ which is known from the previous analysis. $\theta_{B}^{\prime}$ is related to the known direction of the flow in region $\mathrm{A}\left(\theta_{A}^{\prime}\right)$ and the turning angle across the expansion wave $\left(\theta_{A B}\right)$ through $\theta_{B}^{\prime}=\theta_{A}^{\prime}+\theta_{A B} . \theta_{A B}$ is calculated by using Prandtl-Meyer function (Eq. 3.6), which in turn depends on the Mach numbers in region $\mathrm{A}\left(M_{A}\right)$ and $\mathrm{B}\left(M_{B}\right) . M_{A}$ is known, while $M_{B}$ is what needs to be found. 
At this point $\theta_{C}$ has been made a function of $M_{B}$ and thus Eqs. 3.45 and 3.47 are a set of two equations and two unknowns. Due to the nature of the Eqs. 3.6, 3.47 and 3.45 the equations have to be solved simultaneously through iterations. Table 3.1 contains a summary of the angles used to find the direction of the expansion wave from region $\mathrm{A}$ to $\mathrm{B}\left(\tau_{B}^{\prime}\right)$ and the direction of the flow in region $\mathrm{B}\left(\theta_{B}^{\prime}\right)$.

\begin{tabular}{|c|c|c|}
\hline Symbol & Meaning & Obtained from \\
\hline$\beta_{A}$ & Shock angle between A and D (wrt flow in A) & $\beta_{r}$ (Section 3.5) \\
$\beta_{B}$ & Shock angle between B and C (wrt flow in B) & Eqs. 3.45, 3.46 and 3.47 \\
$\tau_{B}$ & Expansion wave angle from A to B (wrt flow in B) & $\sin ^{-1}\left(1 / M_{B}\right)$ \\
$\tau_{B}^{\prime}$ & Expansion wave angle from A to B (wrt horizon) & $\tau_{B}+\theta_{B}^{\prime}$ \\
$\tau_{E}$ & Expansion wave angle from D to E (wrt flow in E) & $\sin ^{-1}\left(1 / M_{E}\right)$ \\
$\tau_{E}^{\prime}$ & Expansion wave angle from D to E (wrt horizon) & $\tau_{E}+\theta_{E}^{\prime}$ \\
$\theta_{A}^{\prime}$ & Direction in region A (wrt horizon) & $\theta_{i}($ Section 3.5) \\
$\theta_{B}^{\prime}$ & Direction in region B (wrt horizon) & $\theta_{A}^{\prime}+\theta_{A B}$ \\
$\theta_{C}$ & Direction in region C (wrt flow in B) & $\theta_{C}^{\prime}+\theta_{B}^{\prime}$ and Eq. 3.47 \\
$\theta_{C}^{\prime}$ & Direction in region C (wrt horizon) & $\theta_{E}^{\prime}$ \\
$\theta_{D}^{\prime}$ & Direction in region D (wrt horizon) & $\theta_{s s}$ (Section 3.5) \\
$\theta_{E}^{\prime}$ & Direction in region E (wrt horizon) & $\theta_{c b}$ (Section 3.5) \\
$\theta_{A B}$ & Turning angle from A to B & $\nu_{B}-\nu_{A}$ (Eq. 3.6) \\
\hline
\end{tabular}

Table 3.1: Summary of angles used for the shock-expansion wave interaction calculations.

$\tau_{B}^{\prime}$ is used to find the location on the cowl surface from which the expansion wave emanates while $\theta_{R}^{\prime}$ is needed to check if this location is correct. $\theta_{B}^{\prime}$ should match the slope of the cowl surface at the expansion wave $\left(\theta_{C: W}\right.$ in Figure 3.16) when the correct size for the Mach stem has been found. As can be seen from the analysis presented above to find the correct size of the Mach sten, and therefore the actual geometry of the cowl shock, iterations are required. The iteration process is started at the point on the initial cowl shock next to the surface of the centre body. Marching through 
the points above it the process is repeated until the correct Mach stem size is found.

The following is a summary of the preceding Mach stem analysis. The initial cowl shock shape is estimated (Eq. 3.27) based on the Mach number at the leading edge of the cowl $\left(M_{1}(x . r)\right)$ and the flow properties after the shock are calculated. Assuming the location of the triple point(Figure 3.14) the direction of the slipstream $\left(\theta_{s s}\right.$ or $\left.\theta_{D}^{\prime}\right)$ and the reflection shock $\left(\beta_{r}\right.$ or $\left.\beta_{A}\right)$ are obtained. Using the direction of the slipstream and the properties of the flow right after the Mach stem the location and the size of the throat are calculated $\left(x^{*}\right.$ and $\left.A^{*}\right)$. Using the throat location the direction of the flow through the throat $\left(\theta_{r h}\right.$ or $\theta_{F}^{\prime}$ ) (parallel to the surface of the centre body at throat location) is obtained, which is used to find the location of intersection of the reflection shock and the expansion wave emanating back from the throat location. This information is required to check if the assumed triple point location (i.e. the height of the Mach stem) is correct. Assuming the shock angle of the reflection shock between region $\mathrm{B}$ and $\mathrm{C}\left(\beta_{B}\right)$, the direction of the expansion wave $\left(\tau_{B}\right)$ as well as the direstion of the flow in regions $\mathrm{B}\left(\theta_{B}^{\prime}\right)$ and $\mathrm{C}\left(\theta_{C}^{\prime}\right)$ are computed. If the direction of the flow in region $\mathrm{C}\left(\theta_{C}^{\prime}\right)$ does not match the direction of the flow in region $\mathrm{E}\left(\theta_{E}^{\prime}\right)$ the previous step is repeated with a new value of the shock angle $\left(\beta_{B}\right)$. Once $\theta_{E}^{\prime}=\theta_{C}^{\prime}$ the direction of the fluw in region $\mathrm{B}\left(\theta_{B}^{\prime}\right)$ is compared to the slope of the cowl at the point where the expansion wave intersects it $\left(\theta_{C W}\right)$. If the two match, then the size of the Mach stem is correct, otherwise, the process for the Mach stem is repeated with a new location for the triple point. Once a viable location of the triple point is found, the geometry of the cowl shock as well as the flow properties after the cowl shock are adjusted to incorporate the effect of the Mach stem. Afterwards the flow properties after the cowl shock are averaged in the radial direction for output. 


\subsection{Mass Flow Rate Estimation}

Figure 3.18 shows all the required parameters needed to calculate the mass flow rate through the EI. The idea behind this analysis is that there exists a streamline that after passing through the cowl shock will intersect the cowl surface at a right angle. Above this streamline the flow passes around the cowl and flows outside the EI, while the flow below the streamline passes through the EI. Using this fact it is possible to

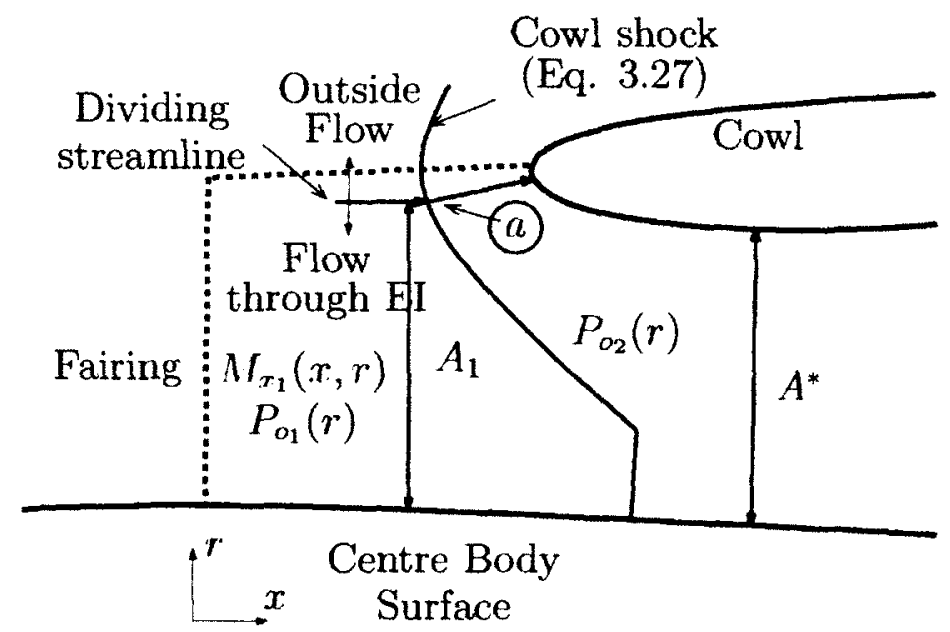

Figure 3.18: Variables needed for the calculation of the mass flow rate through EI.

calculate the mass flow rate through the EI. The streamline is found based on the calculations of the Mach number and its components $\left(M_{x}\right.$ and $\left.M_{r}\right)$ behind the cowl shock (Section 3.5). The data is interpolated until a streamline that intersects the cowl surface at 90 degrees is found. This streamline is the dividing line below which the flow goes through the EI. From the point where this streamline intersects the cowl shock (point a) a vertical line is drawn to the centre body $\left(A_{1}\right)$. This line indicates the area through which the flow passes before it enters the EI. The properties of this flow $\left(M_{x_{1}}\right.$ and $\left.P_{o_{1}}\right)$ can be interpolated from the flow field calculated in Section 3.4 (only $\mathrm{x}$ component of the Mach number is needed in this case). The mass flow rate. can be calculated using Eq. 3.5. However, since the flow properties vary along the 
line, Eq. 3.5 is numerically integrated along $A_{1}$ which results in Eq. 3.48. $M_{i}, P_{o i}$ and $A_{i}$ are defined by Eqs. 3.49, 3.50 and 3.51. In Eq. $3.51 \alpha_{i}$ is length of the circumference in radians available to the flow at radius $r_{i}$ (that is not blocked by the fairing) both of which are shown in Figure 3.19 for area $A_{i}$. The values of $M_{x_{1} i}$ and $P_{o_{1} i}$ are extracted directly from the flow field at radius $r_{i}$, while the value of $\alpha_{i}$ is based on the interpolation from the geometry for a given $x$ and $r_{i}$ values. The $x$ coordinate is the same for all points and is equal to $x$ coordinate of point $a$ which is the location where the dividing streamline intersects the cowl shock (Figure 3.18).

$$
\begin{gathered}
\dot{m}=\sqrt{\frac{\gamma}{R_{a i r} T_{o}}}\left(\sum_{i} M_{i} A_{i} P_{o i}\left(\frac{2}{2+(\gamma-1) M_{i}^{2}}\right)^{\frac{\gamma+1}{2(\gamma-1)}}\right) \\
M_{i}=\frac{M_{x_{1} i}+M_{x_{1} i+1}}{2} \\
P_{m}=\frac{P_{o_{1} i}+P_{o_{1}+1}}{2} \\
A_{i}=\left(r_{i+1}^{2}-r_{i}^{2}\right)\left(\alpha_{i+1}+\alpha_{i}\right) / 4
\end{gathered}
$$

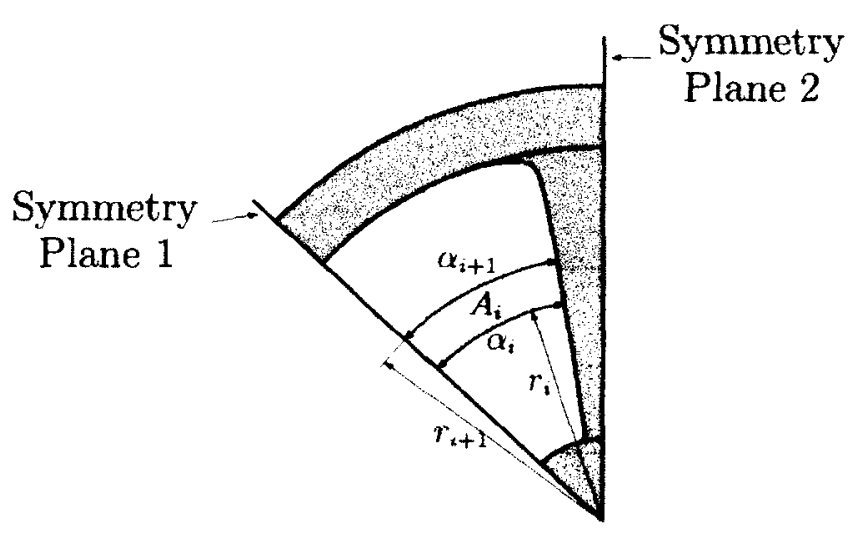

Figure 3.19: Half-section with the variables for area calculations. 
To make sure that the calculated value of $\dot{m}$ does not exceed the choked mass flow rate $\left(\dot{m}^{*}\right)$, the choked condition is calculated and the lesser of the two is used for the final result. Eq. 3.52 is used to calculate $\dot{m}^{*}$ where $A^{*}$ is the minimum cross sectional area of the air passage through the EI taking into account the blockage by the fairings, and $P_{\boldsymbol{O}_{2} a v}$ is the averaged total pressure after the cowl shock which is calculated in Section 3.5 .

$$
\dot{m}^{*}=\sqrt{\frac{\gamma}{R_{a i r} T_{o}}}\left(A^{*} P_{o_{2} a v}\left(\frac{2}{2+(\gamma-1)}\right)^{\frac{\gamma+1}{2(\gamma-1)}}\right)
$$

For the cases where the cowl shock is not computed (e.g. the flow is subsonic after the fairing shock) the $i n$ is calculated for the location at the entrance plane of the EI (i.e. the point $a$ is assumed to be located at the leading edge of the cowl). The $P_{o_{2} a v}$ in Eq. 3.52 is also calculated at the entrance of the EI.

With this the calculations for the shock structure around the EI at the supersonic flight conditions are complete. Along with the shock structure, the preceding analysis allows to find an average $P_{o}$ entering the mixing duct, as well as an estimation of the mass flow rate entering the engine to be calculated. The estimation method takes less than 3 seconds to perform this analysis on a $3.0 \mathrm{GHz}$ processor which is significantly faster than a full 3D simulation. 


\section{Chapter 4}

\section{D CFD simulations}

\subsection{D geometry}

To verify the previously described model for shocks generated by blunt objects, 2D simulations are performed using different Mach numbers and different variations of geometries similar to those discussed. The results are also compared to Billig approximations [28].

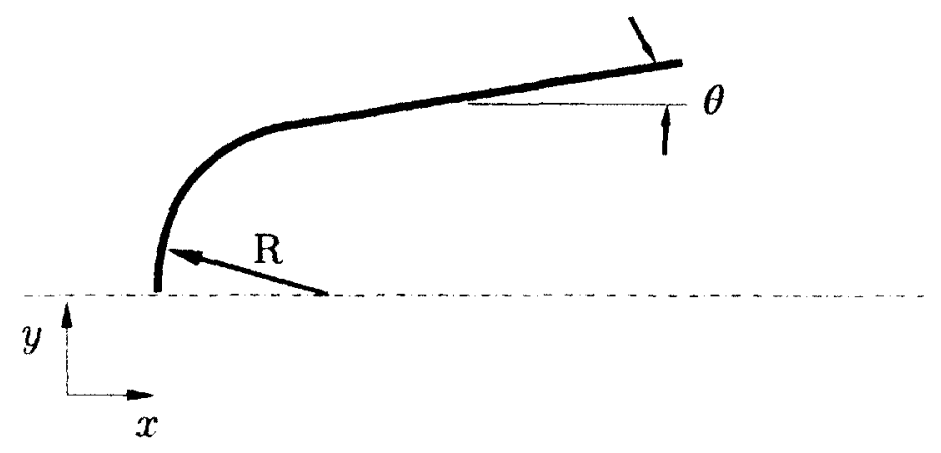

Figure 4.1: Simple 2D geometry.

The schematic of the simulated geometry is shown in Figure 4.1, where $R$ represents the radius of the leading edge and $\theta$ represents the angle at which the surface leaves the leading edge. The radius can be used as a scaling factor for coordinates, meaning it is possible to relate the flow fields for different $R$. This chapter presents 
results for simulations with $\theta$ values of 0,5 , and 20 degrees at various Mach numbers. The simulations are performed using Ansys-CFX software. The following sections describe the setup of the simulations as well as the results of these simulations.

\subsection{Domain, Mesh and Simulation setup}

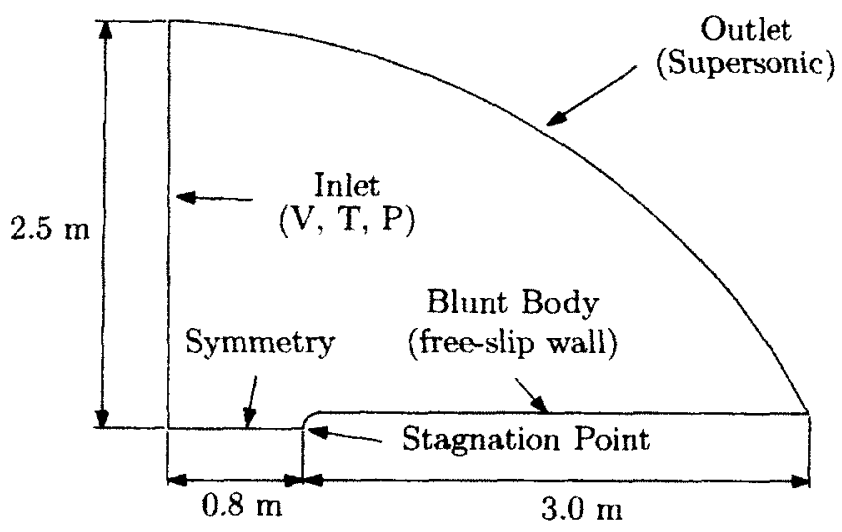

Figure 4.2: $2 \mathrm{D}$ domain with boundary conditions shown $(\mathrm{R}=0.1 \mathrm{~m})$.

Figure 4.2 shows the domain used for the $2 \mathrm{D}$ simulations. The shape of the domain is dictated by the desire to use the same mesh for different conditions, while trying to keep the number of unneeded elements to a minimum. This shape also ensures that the shock does not reflect from any boundary. The mesh used for all $2 \mathrm{D}$ simulations is structured, with the element size near the stagnation point kept at approximately $0.1 \mathrm{~mm}$ (depending on the mesh size).

Figure 4.2 also indicates the boundary types applied to all the surfaces. All simulations are performed with inlet static temperature set to $300 \mathrm{~K}$, and the static pressure set to $1 \mathrm{~atm}$. The tested Mach numbers are $1.1,1.3,1.5,2.0,2.5,3.0$. The outlet is set as a supersonic outlet and thus does not require any additional parameters. The symmetry plane and the side walls of the domain are set to symmetry. The symmetry conditions on the side walls of the domain are required by Ansys-CFX to keep the thickness of the mesh to a single element. The wall of the blunt object 
is set to free-slip wall which means that the viscous boundary layers at the wall are ignored, which simplifies the analysis and reduces the computational time.

The size of the domain is consistent in all cases tested with a height of $2.5 \mathrm{~m}$ and a length of $3.8 \mathrm{~m}$ (Figure 4.2). The simulations are performed for geometries with $R=0.1 \mathrm{~m}$ and $R=0.01 \mathrm{~m}$. The change in $\mathrm{R}$ allows for minimum changes to mesh size and the overall domain, while accomplishing two objectives. The larger radius $(R=0.1 \mathrm{~m})$ allows a high resolution of the shock near stagnation point. The smaller radius $(R=0.01 \mathrm{~m})$ allows for analysis of the shock geometry in the region further away from the blunt object. This is because as indicated in Section 3.4, both stand-off distance and radius at the shock vertex are non-dimensionalized by the radius of the blunt end. The geometry with $R=0.1 \mathrm{~m}$ provides high resolution when non-dimensionalized using $x_{e} / R=0.001$ and thus yields both a more precise location of the shock and more precise radius of the shock at the vertex. In this case, $x_{t}$ is the fixed element size in the stagnation region. The geometry with $R=0.01 \mathrm{~m}$ yields information about the shock away from the geometry up to $x_{\text {domain }} / R=300$ (as measured from the stagnation point).

Figure 4.3 shows an example of meshes for $R=0.01 \mathrm{~m}, \theta=0$ deg and $\theta=20 \mathrm{deg}$. Even though the viscous boundary layers are ignored the velocity gradients near the wall of the object are still relatively high due to the effect of the bow shock, as such the mesh is refined close to the wall. Additional refinement is also done in the region around the stagnation point to resolve the high gradients in that region due to the flow deceleration and the shock.

The advection scheme is set to the first order upwind scheme. The main reason for not using a second order scheme is the means by which the location and the shape of the shock is found. To locate the shock an extraction algorithm determines the location of the largest magnitude of local Mach gradient along a chosen set of streamlines. For high Mach numbers this is generally the correct location of the shock, 


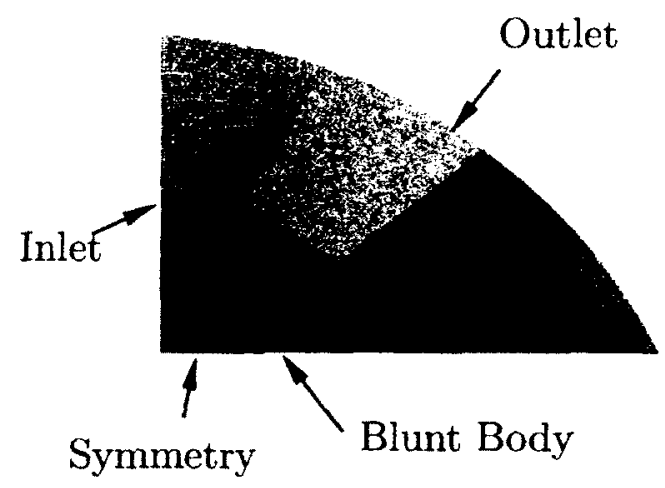

(a) $\theta=0 \mathrm{deg}$

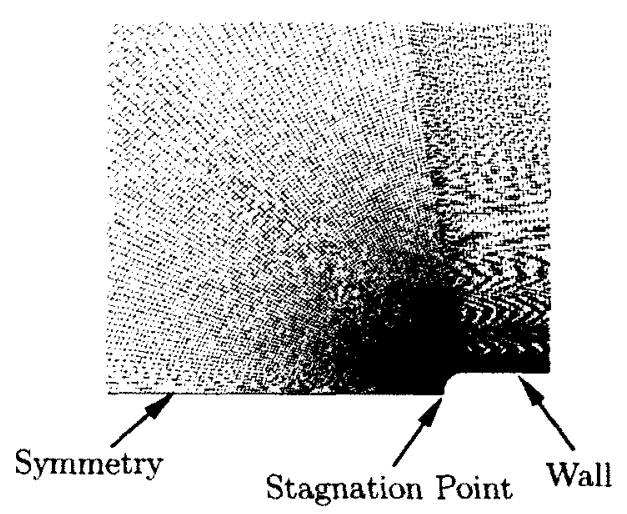

(c) Stagnation region $(\theta=0 \mathrm{deg})$

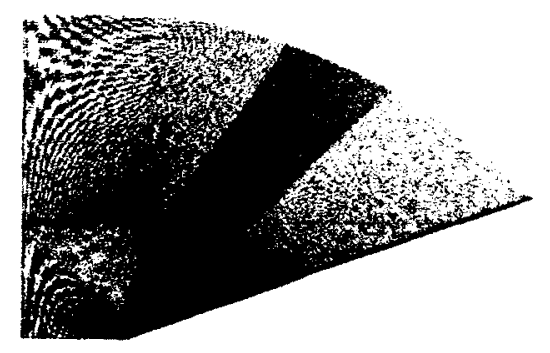

(b) $\theta=20 \mathrm{deg}$

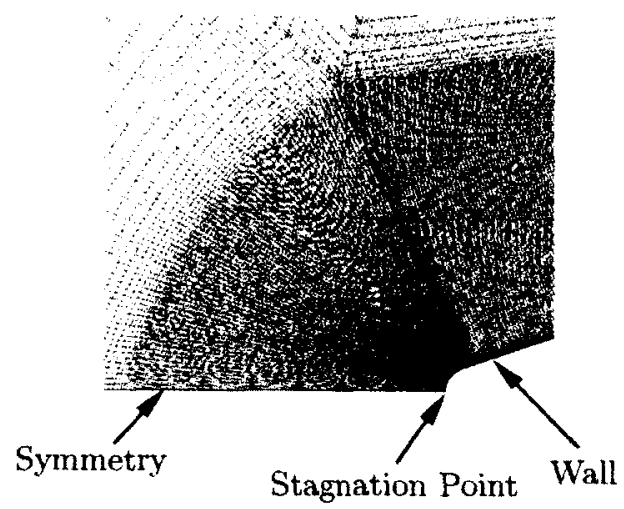

(d) Stagnation region $(\theta=20 \mathrm{deg})$

Figure 4.3: Examples of the mesh for 2D CFD simulations $(R=0.01 \mathrm{~m})$.

however, for lower speeds $\left(M_{\infty} \leq 1.5\right)$ this is not necessarily the case because of the low shock strength. Figure 4.4 shows that at lower Mach numbers the deceleration seen at the stagnation point can lead to a higher calculated gradient than at the shock. This can be compared to the results obtained for $M_{\infty}=2.0$ (Figure 4.8) where the shock produces the largest gradient of the Mach number. As a result the extraction algorithm must search for the first local maximum in the Mach gradient.

Ansys-CFX has a few advection schemes available, though of interest are only two of them: first order upwind and high resolution (HR) schemes [24]. The HR scheme uses a blend factor to switch between first order upwind scheme in the locations of high gradients (e.g. shocks) to second order central scheme everywhere else. As a 


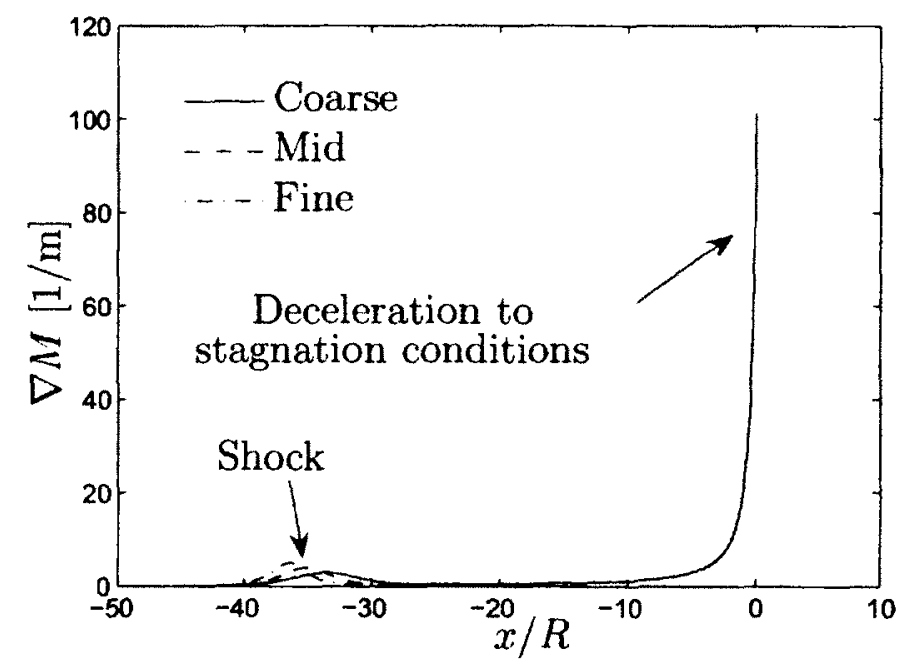

Figure 4.4: Mach gradient distribution along the symmetry plane for different mesh sizes $\left(M_{\infty}=1.1\right)$.

result HR scheme is more accurate than the first order upwind scheme. The second order advection schemes are observed to produce unrealistic numerical oscillations in the regions close to high gradient which is the reason why the HR scheme tries to switch to the first order upwind scheme in such regions. This helps to reduce the oscillations, however, it does not eliminate them. If the HR scheme is used, the shock extraction algorithm can mistake these oscillations for the shock. To avoid these possible errors the HR scheme is not used, instead. first order upwind scheme is used for all of the 2D simulations, even though the HR scheme is more accurate overall for the same mesh.

Due to the presence of the detached shock the "initialization" of the domain is performed in two steps using a very coarse mesh (29880 elements): run a transient simulation for a very short period of simulated time $(1 \mathrm{~ms})$, followed by a steady state simulation until convergence, after which the desired simulations are started. The transient simulation ensures the convergence of the following steady state simulation by calculating the shock in the immediate vicinity of the blunt leading edge. Steady state simulations were found to diverge in a few iterations without this step. 
The steady state simulations are employed as they converge faster than a continuous transient simulation to yield a reasonable initial flow field for more detailed calculations. In the available literature, some authors note that steady state simulations do not provide the correct position for the shocks and as such transient or explicit simulations are required [30].

After this initialization process the final simulation is performed using a transient analysis with initial values obtained from the initialization procedures described above. This final transient simulation is run for $0.05 \mathrm{~s}$ of simulated time using double precision (single precision is used for the initialization procedure). Figure 4.5 shows the residual values for one of the runs. As seen from the figure the numerical convergence is achieved after $0.02 \mathrm{~s}$. Despite this, $0.05 \mathrm{~s}$ of simulated time is used to allow for possible variations in the initialization runs, and allow more time for the residual to converge for higher Mach number simulations.

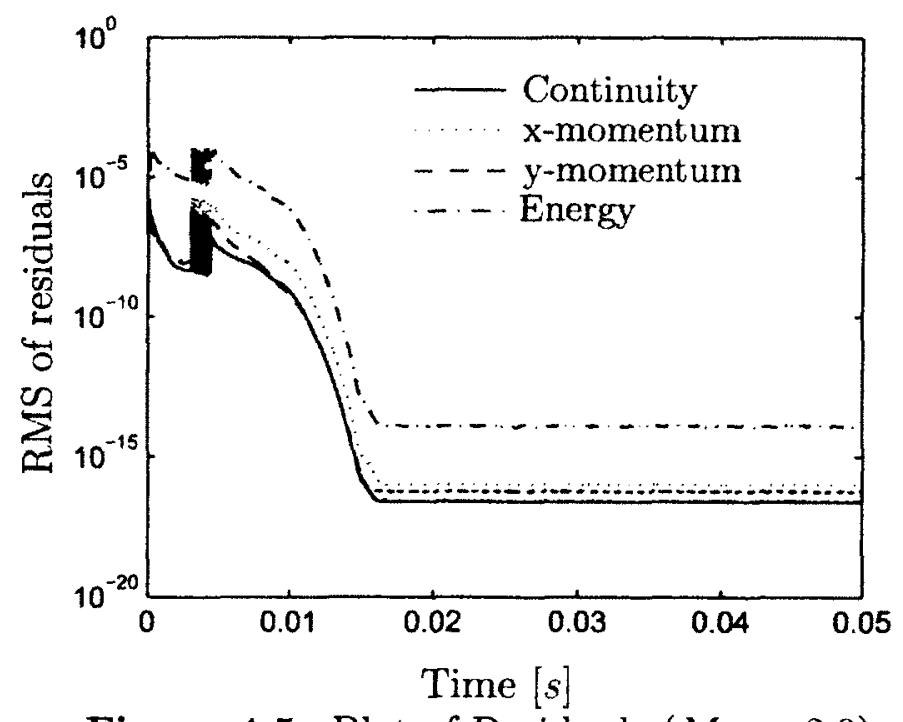

Figure 4.5: Plot of Residuals $\left(M_{\infty}=2.0\right)$.

To find an optimum mesh size a grid sensitivity study is performed and the results are presented in the section below. 


\subsection{Grid Sensitivity Study}

The effect of the mesh size is tested for two different inlet conditions corresponding to $M_{\infty}=1.1$ and $M_{\infty}=2.0$. In both cases the deflection angle is set to 0 . The reason for two different tests is that the shock geometries at these two conditions differ considerably. The tested mesh sizes are presented in Table 4.1 where each successive mesh approximately doubles in node count. The effect of the different mesh sizes on the stand-off distance is shown in Figure 4.6. In addition to comparing the stand-off distance, the Mach number distribution (Figures 4.7a and b) and its gradient (Figures 4.4 and 4.8 ) along the symmetry plane are also compared .

\begin{tabular}{|c|c|c|c|c|}
\hline Mesh & $\begin{array}{c}\text { Number of } \\
\text { Elements }\end{array}$ & $\begin{array}{c}\text { Number of } \\
\text { Nodes }\end{array}$ & $\begin{array}{c}\text { Computational } \\
\text { Time }[h r]\end{array}$ & $\begin{array}{c}\text { Relative increase } \\
\text { in mesh size }\end{array}$ \\
\hline Coarse & $1.2 \times 10^{5}$ & $2.4 \times 10^{5}$ & 10 & - \\
Mid & $2.6 \times 10^{5}$ & $5.3 \times 10^{5}$ & 25 & 2.2 \\
Fine & $5.4 \times 10^{5}$ & $10.9 \times 10^{5}$ & 53 & 2.1 \\
\hline
\end{tabular}

Table 4.1: List of meshes for $2 \mathrm{D}$ simulations.

\begin{tabular}{|c|c|c|}
\hline & \multicolumn{2}{|c|}{$\%$ difference in $d_{s} / R$} \\
\hline Mesh & $M_{\infty}=1.1$ & $M_{\infty}=2.0$ \\
\hline Coarse & 4.3 & 1.25 \\
Mid & 3.7 & 0.85 \\
Fine & - & - \\
\hline
\end{tabular}

Table 4.2: Grid convergence summary.

As seen from Table 4.2 and Figure 4.6 the shock stand-off distance is not significantly affected by the mesh size. This is especially true for $M_{\infty}=2.0$ simulations where the difference in $d_{s} / R$ between the coarse and mid mesh is $1.25 \%$ and the difference between the mid and fine meshes is $0.85 \%$. The mesh size and computation time for the mid mesh on the other hand is twice that of the coarse mesh. Same 


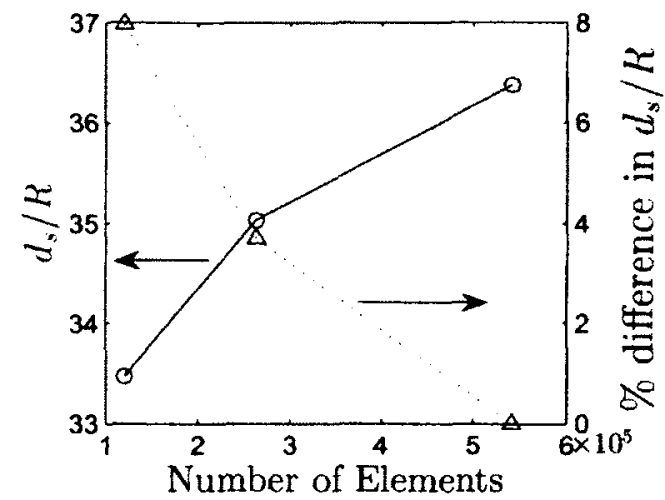

(a) $M_{\infty}=1.1$

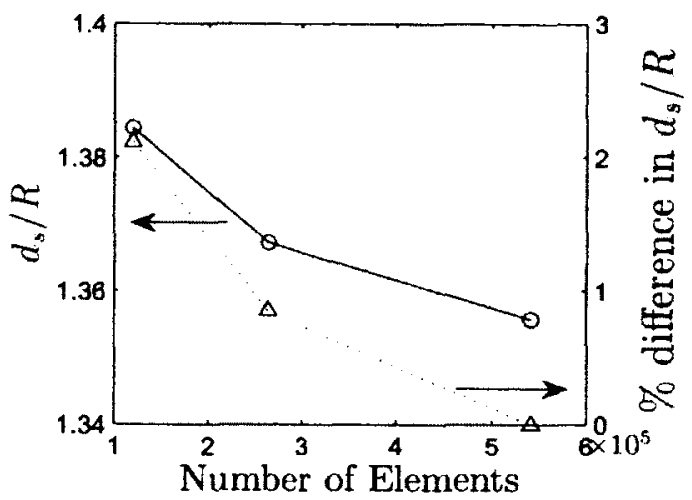

(b) $M_{\infty}=2.0$

Figure 4.6: Stand-off distance as a function of the mesh size.

applies for fine and mid mesh comparison for which the computational time for fine mesh simulations is almost double that of the mid mesh. Based on these results the doubling in mesh size does not yield an appreciable change in $d_{s} / R$ value. In the case of the simulations for $M_{\infty}=1.1$ the differences are larger. The difference in $d_{s} / R$ value between the coarse and mid mesh is $4.3 \%$ and between the mid and fine mesh it is $3.7 \%$. One reason for this larger difference is that at lower Mach numbers the shock stand-off distance is larger, placing the shock in a region where the mesh is considerably coarser with element size of $4 \mathrm{~mm}$ as opposed to $0.1 \mathrm{~mm}$ in the region closer to the stagnation point. This results in lower shock resolution and a greater uncertainty in the shock location.

Figure 4.7 shows the Mach distribution along the symmetry plane for $M_{\infty}=1.1$ (a) and $M_{\infty}=2.0$ (b). As seen from these figures the fine mesh results yield the sharpest discontinuity across the shock in both cases, this result is also seen from the Mach number gradient in Figures 4.4 and 4.8. In the case of $M_{\infty}=1.1$ (Figure 4.7a) the computed shock region is roughly 5 to 10 radii thick, whereas for $M_{\infty}=2.0$ (Figure $4.7 \mathrm{~b}$ ) the shock region is only $0.25-0.5$ radii thick. This reinforces the point about the effect of coarser mesh further away from the blunt body which increases the uncertainty in shock location. Figure 4.8 compares the Mach number gradient along 


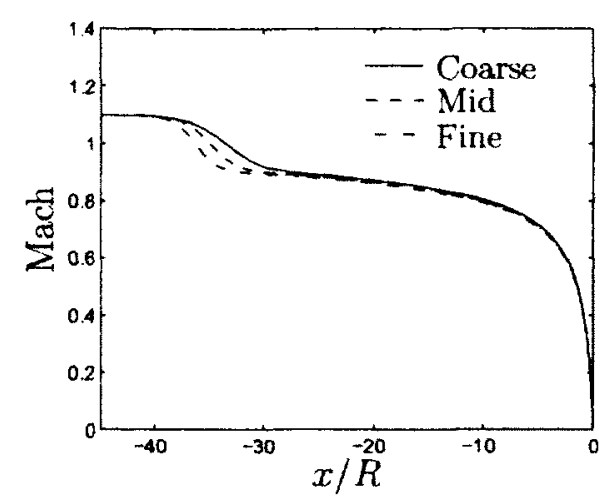

(a) $M_{\infty}=1.1$

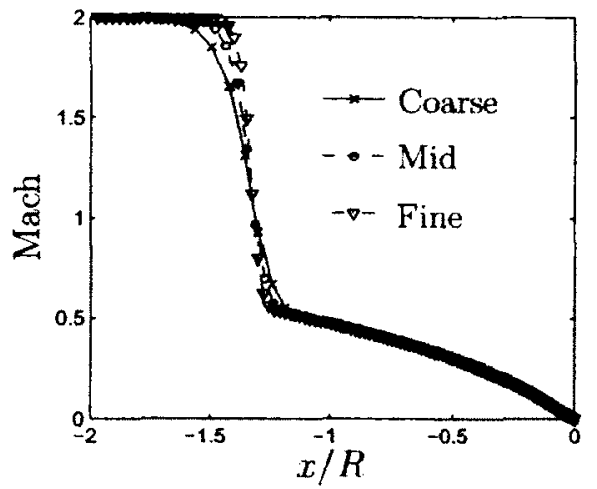

(b) $M_{\infty}=2.0$

Figure 4.7: Mach distribution along the symmetry plane.

the symmetry plane. As seen from the figure the finer mesh shows a considerable increase in the magnitude of the gradient, while the bandwidth of the spiked region is narrower than that of the coarser meshes. This shows that the finer mesh does bring the shock closer to being a discontinuity. Based on these results all further calculations are performed using the mid size mesh (260 000 elements). This mesh produces results that differ by less than $5 \%$ for $M_{\infty}=1.1$ and and less than $1 \%$ $M_{\infty}=2.0$ from the fine mesh and take half the time to complete the calculations when compared to a mesh doubled in size.

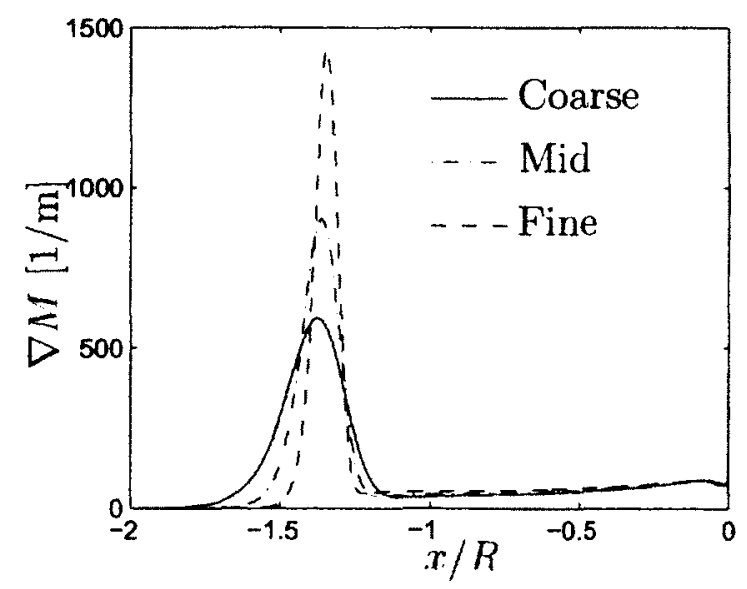

Figure 4.8: Mach gradient distribution along the symmetry plane $\left(M_{\infty}=2.0\right)$. 


\subsection{Results and comparison}

Figure 4.9 shows the plot of the shock stand-off distance $\left(d_{\mathrm{s}} / R\right)$ on the log scale versus $1 / M^{2}$ for CFD results, experimental results [40] and estimated results (Eq. 3.24). As seen from the figure the shock stand-off distance estimated from Eq. 3.24 agrees well with the CFD simulations for $M_{\infty} \geq 1.3\left(1 / M^{2} \leq 0.6\right)$. However, at $M_{\infty}=1.1$ the

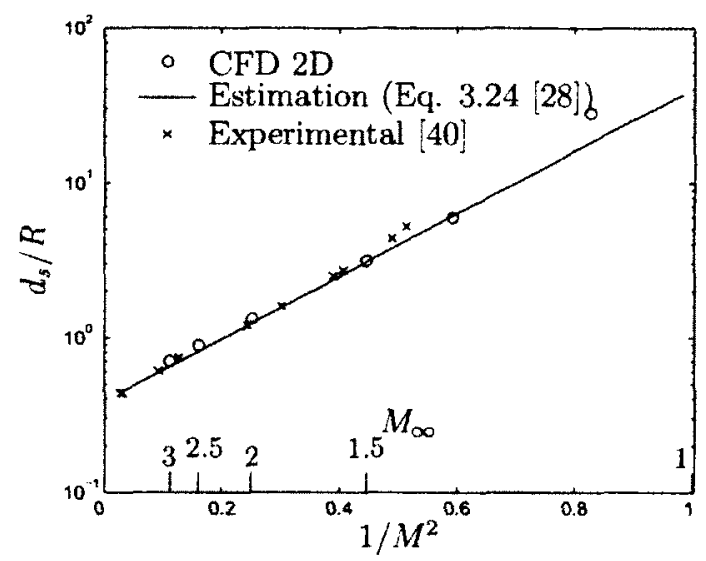

Figure 4.9: Shock stand-off distance vs Mach number.

stand-off distance is underestimated when compared to the numerical simulations. When compared to the experimental results from the literature [40] the stand-off distance approximation agrees well for $M_{\infty}>1.5$ (below $1 / M^{2} \approx 0.45$ ) but again underpredicts the stand-off distance at lower Mach numbers. This means that the curve fit proposed by Billig (Eq. 3.24) is well suited for higher Mach number flows. For the actual EI geometry at high Mach numbers, where multiple shock intersections are more likely, the estimation provided by Eq. 3.24 for the shock stand-off distance is sufficiently accurate. At low Mach numbers a single detached shock is likely sufficient to decelerate the flow to subsonic velocities thus eliminating the possibility of shock intersections. At these low Mach numbers it is the total pressure drop through the shock that is important and not the precise shock location (as there are no downstream interactions), as such the difference between Eq. 3.24 and the experimental or CFD 
results can be neglected.

Figure 4.10 compares shock shapes produced by the blunt object $(\theta=0$ deg $)$ at different Mach numbers as calculated by Ansys-CFX, predicted by Billig (Eq. 3.25), and estimated as described in Section 3.5 (Eq. 3.27). As seen from these results Billig's equation agrees well with the CFD results at higher Mach numbers. However, at lower Mach numbers (e.g $M_{\infty}=1.3$ ) Eq. 3.25 fails to capture the generated shock shape far from the object. This leads to a revision of the equation for the shock shape and $R_{s}$ and results in Eqs. 3.25 and 3.31. According to Figure 4.10, Eq. 3.31 shows much better agreement with the CFD results at all conditions tested.

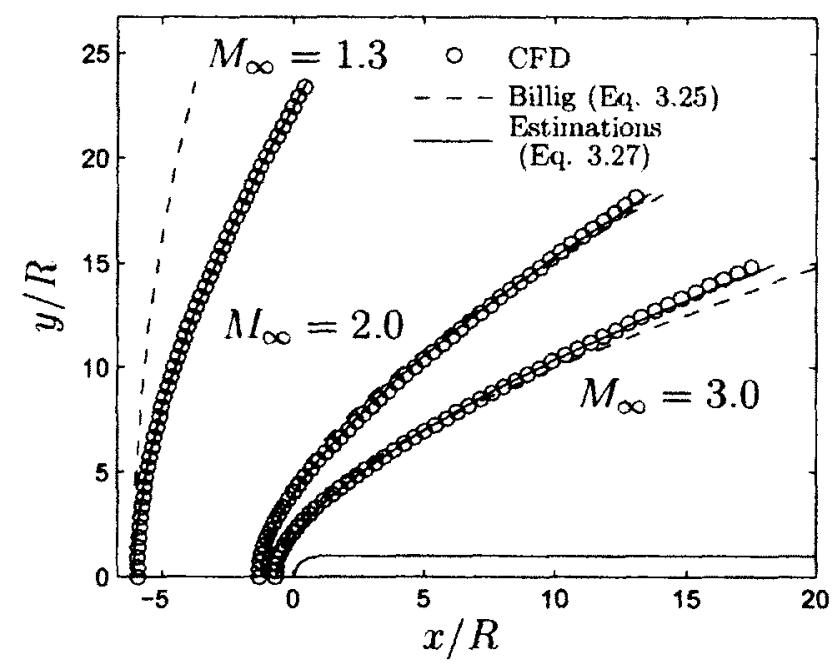

Figure 4.10: Shape of the shocks produced by a blunt body at different Mach numbers $(R=0.1 \mathrm{~m})$.

While Figure 4.10 shows the shock shape close to the object (within 20 radii), Figure 4.11 shows the shock shape as far as 200 radii for the same free stream conditions. Figure 4.11 shows that there is still some discrepancy between the estimated shock shape and the CFD results further away from the object where the estimation method underestimates the shock angle as compared to 2D CFD simulations. This leads to the following analysis.

Figure 4.12 shows the shock shape as computed using the Billig equation (Eq. 


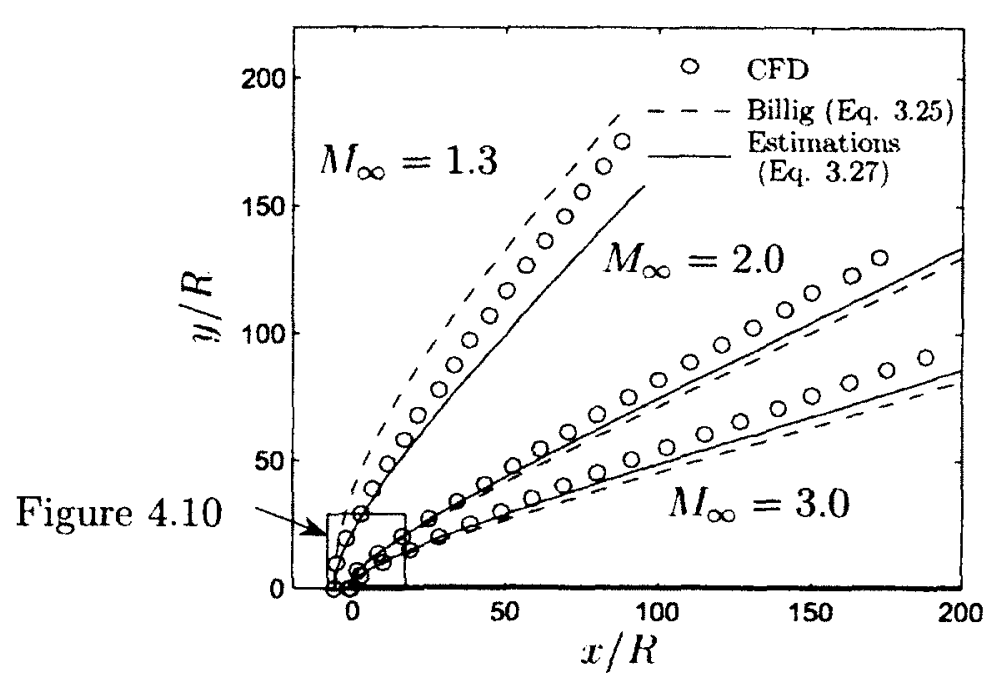

Figure 4.11: Shape of the shocks produced by a blunt body at different Mach numbers $(R=0.01 \mathrm{~m})$.

3.25) and the current estimation (Eq. 3.27) that occurs for a deflection angle of 3 deg, while the CFD results are taken from the same simulations with $\theta=0$ deg. As seen from this figure the estimated shock shape matches the CFD results quite well. However, Figure 4.13 shows that for deflection angles $\theta$ with values of 5 deg and $20 \mathrm{deg}$ the estimated shock shape matches the CFD results quite well without any adjustments. The reason for these differences is due to the expansion of the flow around the leading edge of the object. Figure 4.14 shows the contour plot for the directions of the flow for $\theta=0 \mathrm{deg}$ (a) and $\theta=20 \mathrm{deg}$ (b) cases. In the case of $\theta=0$ deg (Figure 4.14a) part of the flow behind the shock remains nilisaligned with the object surface because some of the expansion waves do not intersect the shock. This misalignment angle according to CFD simulations is around 3 deg which is the adjustment angle in Figure 4.12. In this case the slope of the asymptote of the shock is equivalent to the slope of an attached shock produced by a wedge with deflection angle equal to approximately $3 \mathrm{deg}$. In the case of $\theta=20 \mathrm{deg}$ (Figure 4.14b) the expansion waves intersect the shock within a few radii, which means that the flow further away from the stagnation point is now aligned with object surface and thus 


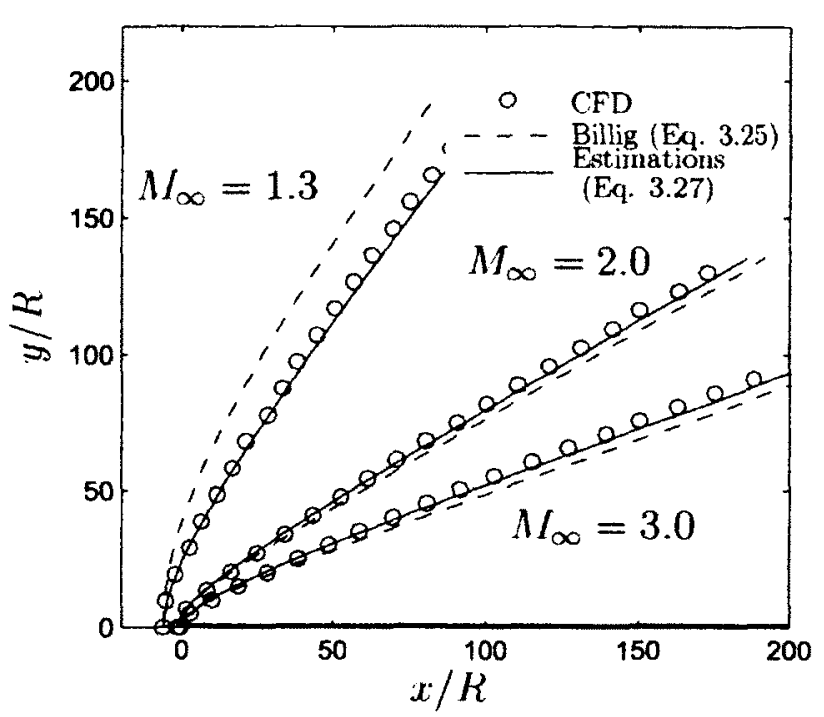

Figure 4.12: Shape of the shocks produced by a blunt body at different Mach numbers, for CFD $\theta=0 \mathrm{deg}$, while for Billig and Estimations $\theta=3$ deg $(R=0.01 \mathrm{~m})$.

the shock shape reaches an asymptotic value consistent with the body angle (Figure $\left.3.10, \beta_{w}=\int\left(\theta_{w}\right)\right)$. Based on this analysis the estimations produce the shock shapes that agree very well with the CFD results and the discrepancy present at $\theta=0$ deg is very small and limited to the flow field further away from the object.

Based on the information provided in this chapter one can conclude that Eq. 3.27 is able to accurately predict the shock shape and position over a variety of Mach numbers and object surface angles $\theta$. With an accurate shock shape, property changes across the shock can then be found allowing an assessment of the overall performance of a given EI. 


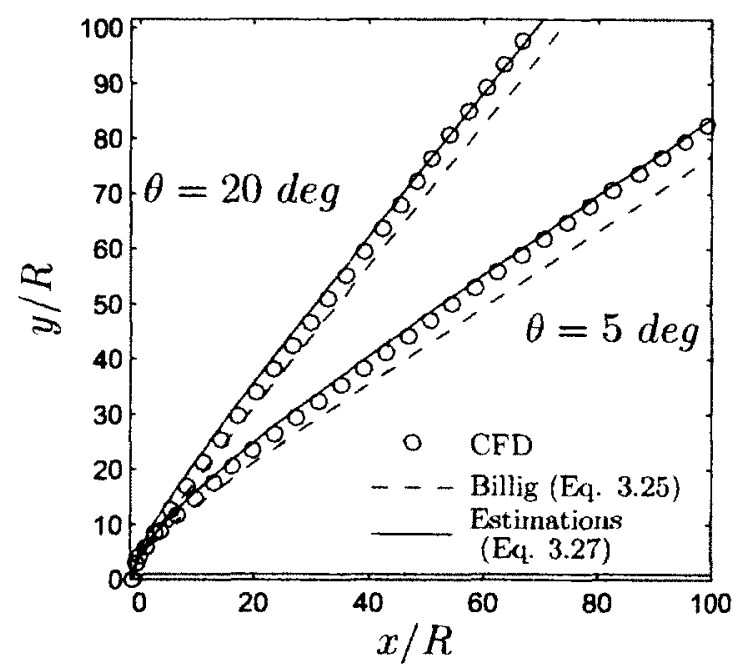

Figure 4.13: Shape of the shocks produced by a blunt body with different deflection angles $\left(R=0.01 \mathrm{~m}, M_{\infty}=2.0\right)$.

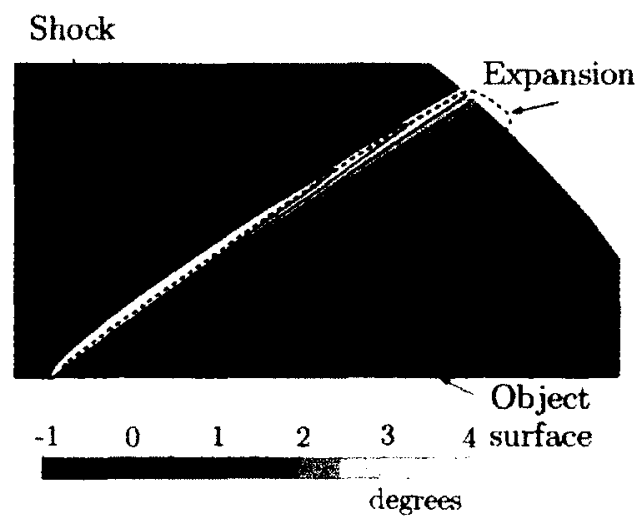

(a) $\theta=0 \mathrm{deg}$
Shock

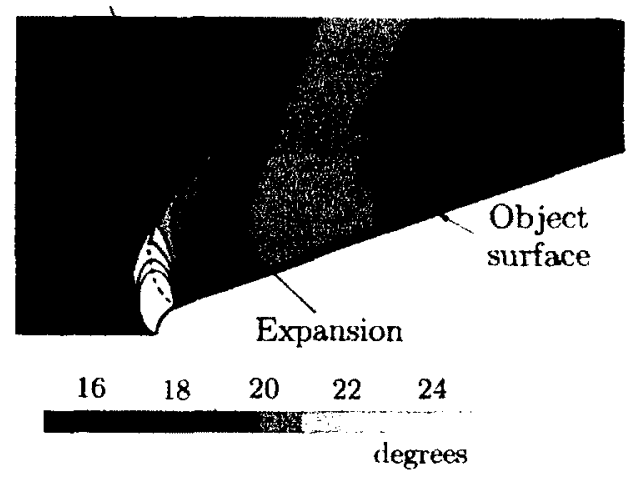

(b) $\theta=20 \mathrm{deg}$

Figure 4.14: Contour plot of the flow direction for $\left(R=0.01 \mathrm{~m}, M_{\infty}=2.0\right)$. 


\section{Chapter 5}

\section{D CFD simulations}

\subsection{Overview}

This chapter describes the 3D simulations of the EI and also compares the results from these simulations to the ones obtained from the estimation method. The comparison is performed for the shock shapes, total pressure drop and the mass flow rate through the EI.

\subsection{D Simulation Setup}

The modelling of the geometry is performed using ICEM CFD software [23]. Due to the symmetry of the RBCC EI only one eighth is modelled. This allows for a finer mesh to be used without excessively raising the computation times. Figure 5.1 shows the section that is being modelled with symmetry planes indicated, while Figure 5.2 shows the computational domain with the boundary conditions. Due to issues with the structured mesh at the nose tip of the centre body the geometry is slightly modified as shown in Figure 5.3. The structured mesh for this analysis is constructed using hexahedron elements. The sharp nose tip of the centre body requires the use of "collapsed" elements, that is one of the faces of these hexahedron 
elements is collapsed to a point becoming a pyramid. However, collapsed elements are not accepted by Ansys-CFX, and as such they cannot be used. Therefore, the tip of the centre body is cut back as shown in Figure 5.3.

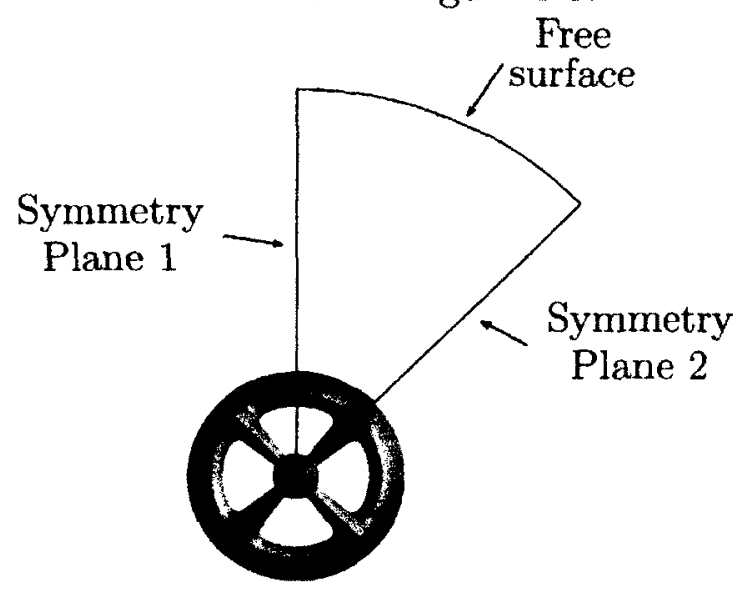

Figure 5.1: Half-section of the EI used for 3D simulations with symmetry planes shown.

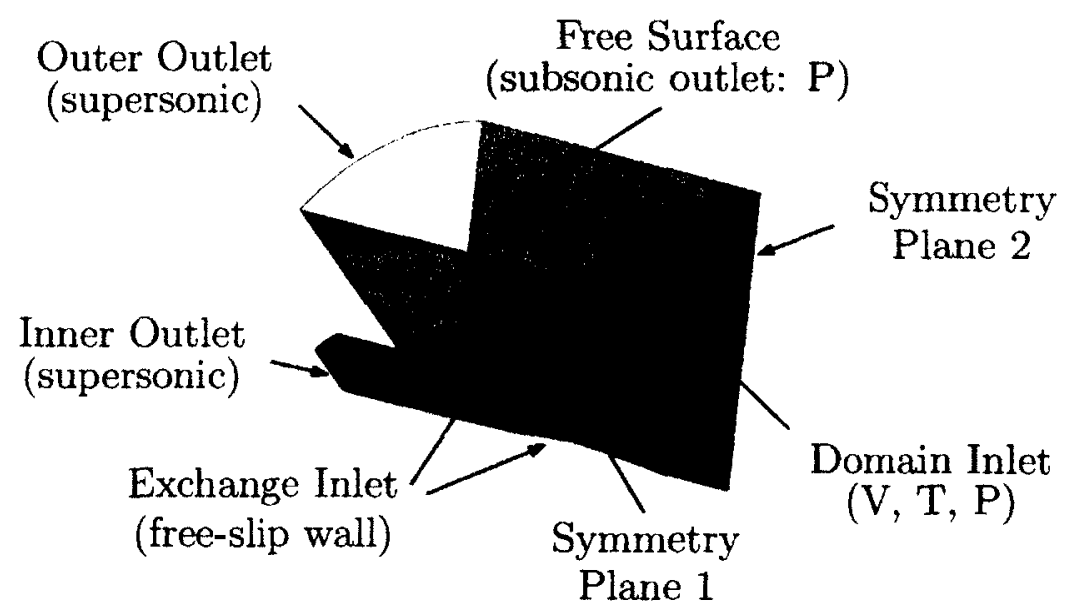

Figure 5.2: The domain setup for CFD simulations with boundaries shown.

Table 5.1 lists the inlet boundary conditions used to obtain the results described in this chapter. These conditions are based on an assumed flight profile as shown in Figure 5.4. This profile is based on a NASA model of atmosphere [41] and a constant dynamic pressure of $30 \mathrm{kPa}$. The criteria for this profile is that it fits into an estimated allowable flight profile for the ramjet and scramjet modes of operation. These limits are also shown in the figure and are based on a papers by Andreadis [42] 


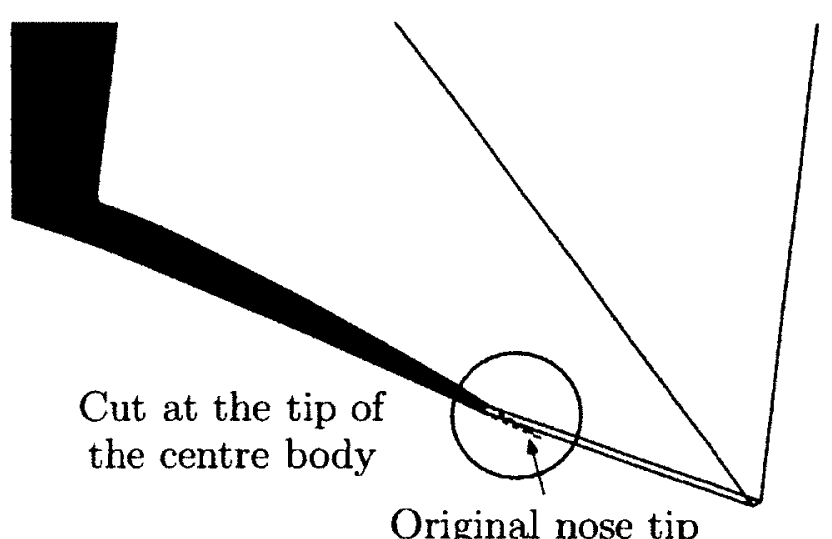

Figure 5.3: Centre body tip geometry.

and Fry [9]. The "Cruise Limit" is set by combustion stability (lower pressure would result in lower combustion efficiency) while the "Structural Limit" is determined by structural and thermal considerations on the likely materials for the engine.

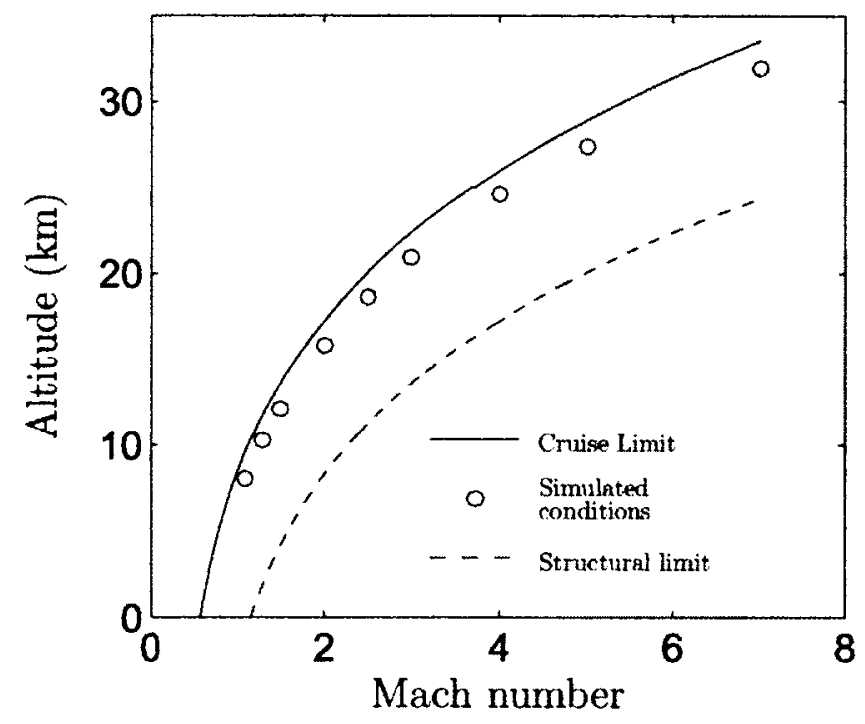

Figure 5.4: Simulated flight profile.

Transient simulations are performed using Ansys-CFX [24] with air modelled as a perfect gas. The reason for choosing a transient analysis is that for this geometry steady state simulations are found to have difficulty converging (as judged by the 


\begin{tabular}{|c|c|c|c|c|c|c|}
\hline Mach & $\begin{array}{c}\text { Altitude } \\
(\mathrm{km})\end{array}$ & $\begin{array}{c}\text { Speed } \\
(\mathrm{m} / \mathrm{s})\end{array}$ & $\begin{array}{c}\text { Static } \\
\text { Pressure } \\
(\mathrm{kPa})\end{array}$ & $\begin{array}{c}\text { Total } \\
\text { Pressure } \\
(\mathrm{kPa})\end{array}$ & $\begin{array}{c}\text { Static } \\
\text { Temperature } \\
(\mathrm{K})\end{array}$ & $\begin{array}{c}\text { Total } \\
\text { Temperature } \\
(\mathrm{K})\end{array}$ \\
\hline 1.1 & 8.1 & 338.7 & 35.4 & 75.6 & 236 & 293 \\
1.3 & 10.3 & 387.8 & 25.4 & 70.3 & 221 & 296 \\
1.5 & 12.1 & 442.6 & 19.0 & 60.9 & 217 & 314 \\
2.0 & 15.8 & 590.2 & 10.7 & 83.8 & 217 & 390 \\
2.5 & 18.6 & 737.7 & 6.9 & 117.2 & 217 & 487 \\
3.0 & 20.9 & 885.3 & 4.8 & 174.9 & 217 & 606 \\
4.0 & 24.6 & 1180.4 & 2.7 & 406.6 & 217 & 910 \\
5.0 & 27.4 & 1499.6 & 1.7 & 906.9 & 224 & 1343 \\
7.0 & 32.0 & 2162.4 & 0.87 & 3620.6 & 237 & 2565 \\
\hline
\end{tabular}

Table 5.1: Flight Conditions.

calculation of an irregular mass flow rate though the inlet). For the results presented in this chapter all of the simulations are initially performed on a very coarse mesh using the initialization approach described in Section 4.2. For these initial simulations the advection scheme is set to first order upwind with the use of single precision as is the case with 2D simulations. All 3D simulations are performed using the second order "High Resolution" advection scheme with double precision. Since unlike the 2D simulations the exact shock location is not extracted based on the Mach gradients.

All of the transient simulations (with the exception of one) are performed for $0.1 \mathrm{~s}$ of simulated time with a time step of $10^{-5} \mathrm{~s}$. These parameters are found to produce stable results for $P_{o}$ and $M$ (i.e. the solution no longer changes with time). The mentioned exception is the fine mesh used in the grid convergence study discussed in the next section. The time step was kept constant for all of the simulations regardless of the inlet conditions. This resulted in a slight variation in the final residuals for different flight conditions. The target for the root mean square (RMS) of the residuals for coefficient loop iteration is set to $10^{-4}$. The coefficient loop is the iteration process 
within a time step.

Figure 5.5 shows the RMS of the residuals for three equations (continuity, momentum in $\mathrm{x}$-direction and energy equations) at each time step. As seen from the figure the RMS drops below the target of $10^{-4}$. After $0.05 \mathrm{~s}$ the change in the residual

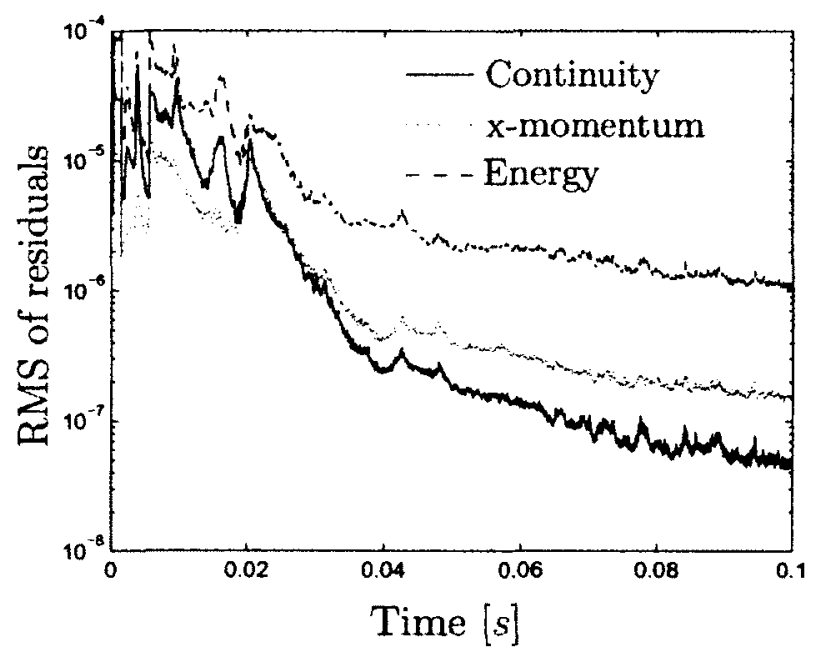

Figure 5.5: Plot of Residuals $\left(M_{\infty}=2.0\right)$.

values becomes relatively small which is indicated by the shallow slope of the RMS values. The simulation is allowed to run until $0.1 \mathrm{~s}$ at which point the residuals remain approximately constant meaning the solution is no longer changing significantly and the simulation is considered to be converged.

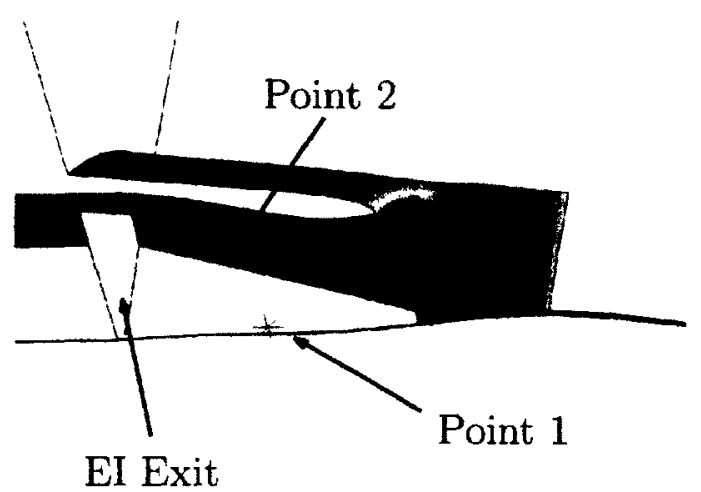

Figure 5.6: Test locations for the time and grid convergence checks. 
Figure 5.7 shows selected variables at different time steps. These variables include Mach number and total pressure at two selected points as well as the mass flow averaged Mach number and total pressure at the exit plane of EI (Figure 5.7a and b) and the mass flow rate through EI (Figure 5.7c). The exit plane and the test points are shown in Figure 5.6. Both points are located on Symmetry Plane 1, with

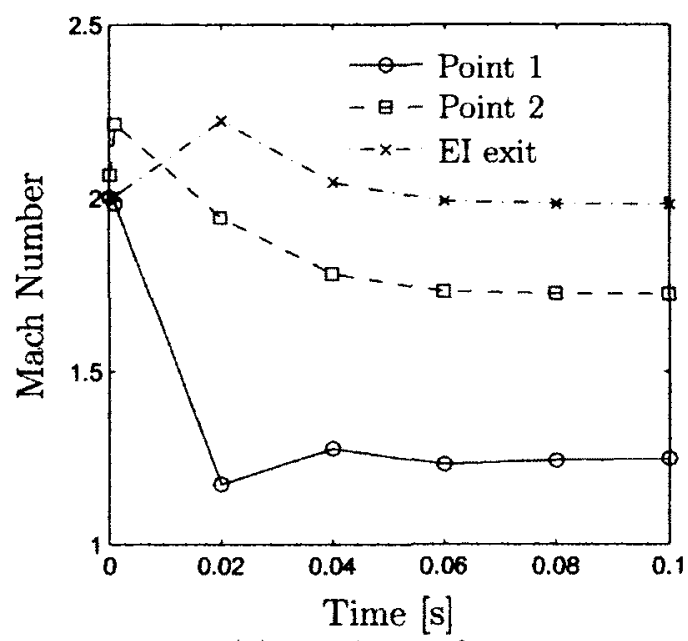

(a) Mach Number

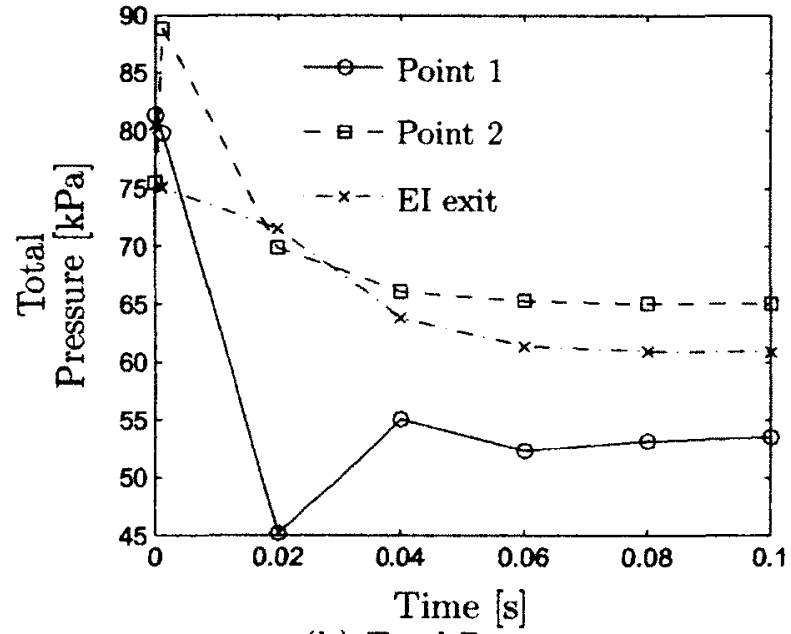

(b) Total Pressure

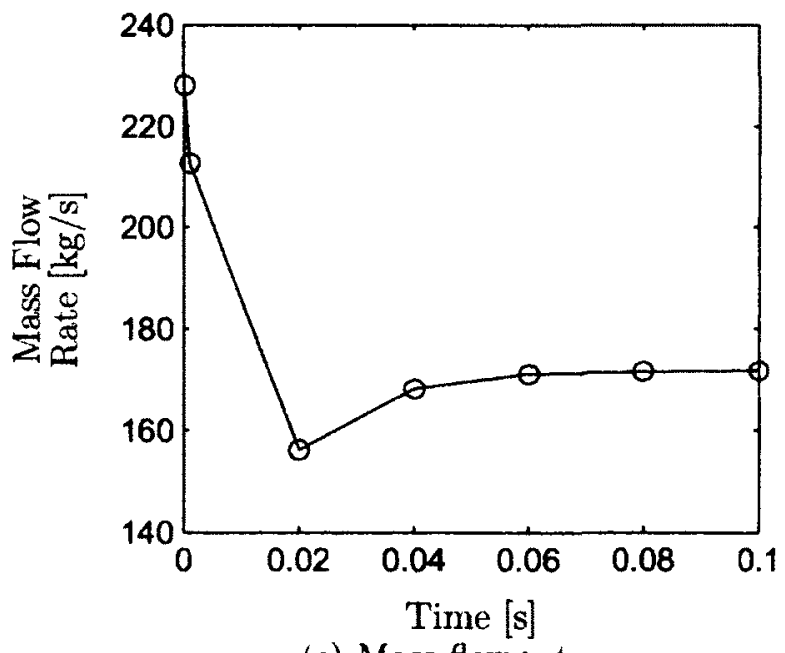

(c) Mass flow rate

Figure 5.7: Time Convergence (Coarse Mesh, $M_{\infty}=2.0$ ).

one being downstream of the Mach stem (Point 1) and the other being downstream of bow section of the cowl shock (Point 2). These points are selected since they are 
located in regions most susceptible to the flow changes. These variables are also the main outputs of the estimation method. As seen from Figure 5.7 all these variables converge to within $2 \%$ of the final values by $0.08 \mathrm{~s}$. This level of convergence is deemed to be sufficient for the purposes of further analysis.

\subsection{Grid sensitivity analysis}

Since there are no experimental data to verify the 3D CFD simulations it is important to reduce as much computational uncertainty as possible and thus a grid sensitivity study is performed. For this case three test meshes were created, plus an additional very coarse mesh, which is used for initialization purposes only. Table 5.2 lists all of the test meshes with the number of elements and nodes within the meshes. The

\begin{tabular}{|c|c|c|c|c|}
\hline Mesh & $\begin{array}{c}\text { Number of } \\
\text { Elements }\end{array}$ & $\begin{array}{c}\text { Number of } \\
\text { Nodes }\end{array}$ & $\begin{array}{c}\text { Computational } \\
\text { Time [days] / } \\
\text { Simulated } \\
\text { Time }[s]\end{array}$ & $\begin{array}{c}\text { Relative increase } \\
\text { in mesh size } \\
\text { (Number of } \\
\text { Elements) }\end{array}$ \\
\hline Coarse & $1.50 \times 10^{6}$ & $1.56 \times 10^{6}$ & $5.3 / 0.1$ & - \\
Mid & $2.71 \times 10^{6}$ & $2.80 \times 10^{6}$ & $10.2 / 0.1$ & 1.8 \\
Fine & $5.27 \times 10^{6}$ & $5.41 \times 10^{6}$ & $12.8 / 0.05$ & 1.9 \\
\hline
\end{tabular}

Table 5.2: List of meshes for $3 \mathrm{D}$ grid convergence study

comparison between the meshes is done for flight conditions corresponding to a free stream Mach number of two. Table 5.2 also indicates the computational time measured in days. The two simulations with the "Coarse" and "Mid" meshes are run until a simulated time of $0.1 \mathrm{~s}$ is reached and are initialized using the results from a steady-state simulation on a very coarse mesh containing $2.1 \times 10^{5}$ elements. This steady-state initialisation simulation takes less than 20 minutes to complete on a single core processor (as opposed to days of transient simulations with the finer meshes on an 8-core computer). As was noted in the previous section the fine mesh simulation 
is not run for a simulated time of $0.1 \mathrm{~s}$. Unlike the coarse and mid mesh simulations the fine mesh simulation is run until a simulated time of $0.05 \mathrm{~s}$ is reached and is initialised using the results from "Mid" mesh. This was done to reduce the computational time, however, as shown in Figure 5.8 the $0.05 \mathrm{~s}$ in this case is sufficient for the simulation to reach a steady-state as previously defined by a fluctuation of less than $2 \%$ on the mass flow rate, Mach number and total pressure at various locations in the EI.

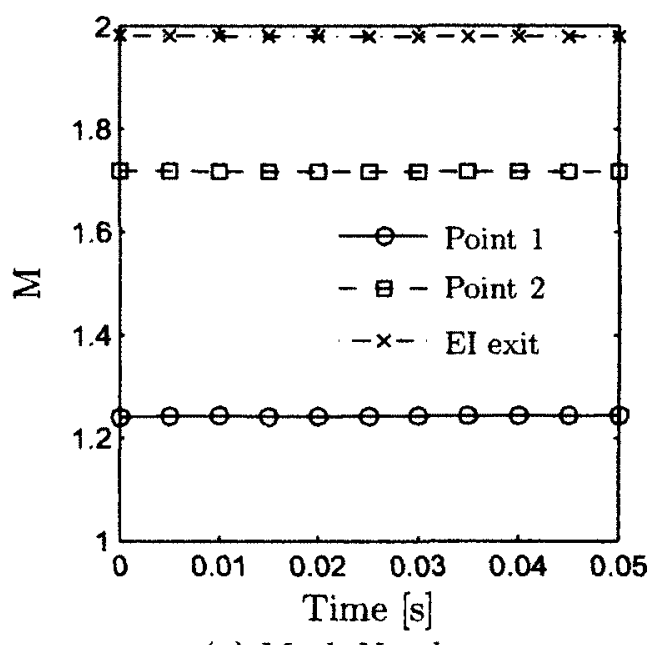

(a) Mach Number

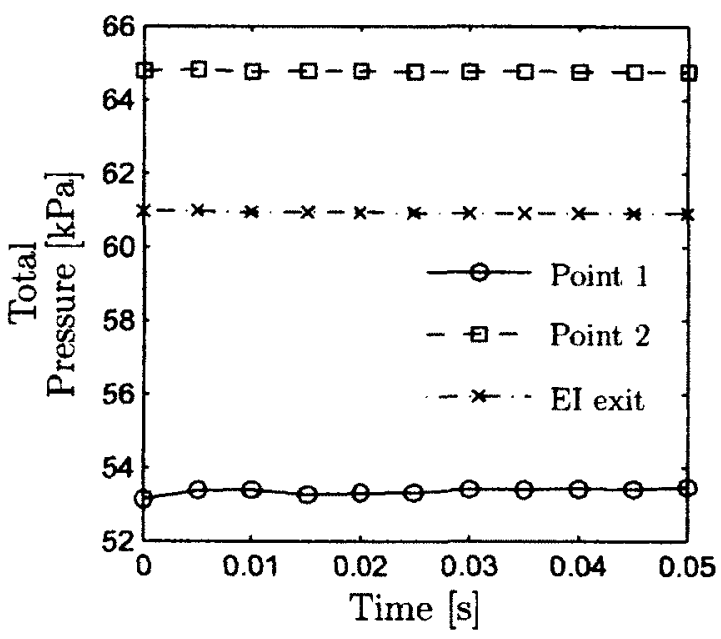

(b) Total Pressure

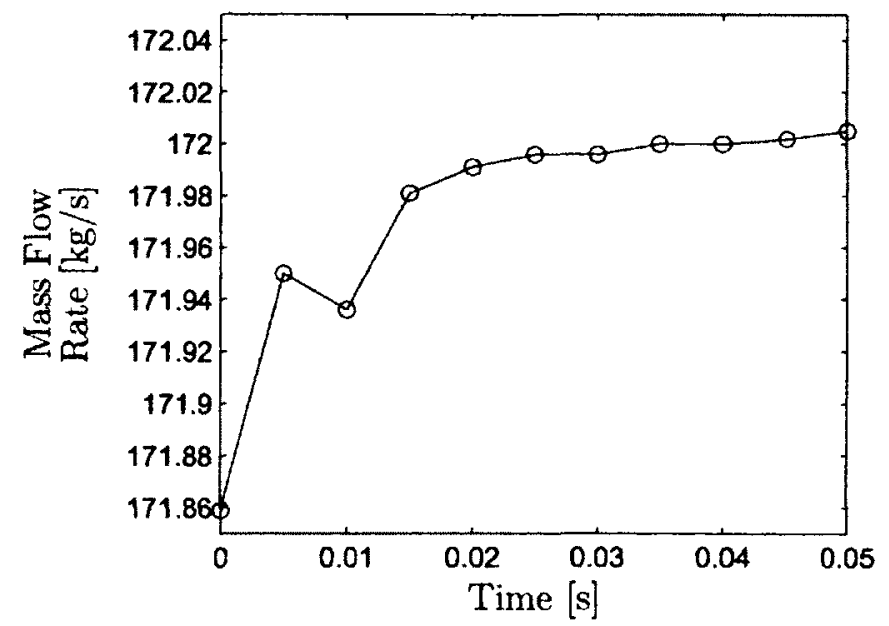

(c) Mass flow rate

Figure 5.8: Time Convergence (Fine Mesh, $M_{\infty}=2.0$ ). 


\begin{tabular}{|c|c|c|c|c|c|c|}
\hline \multicolumn{2}{|c|}{ Mesh: } & \multicolumn{2}{c|}{ Coarse } & \multicolumn{2}{c|}{ Mid } & Fine \\
\hline Variable & Location & Value & \% diff. & Value & \% diff. & Value \\
\hline Total & Point 1 & 53.57 & 2.00 & 52.52 & -1.77 & 53.46 \\
Pressure & Point 2 & 65.10 & 0.31 & 64.90 & 0.20 & 64.77 \\
$(\mathrm{kPa})$ & EI exit & 60.93 & -0.02 & 60.94 & 0.04 & 60.92 \\
\hline Mach & Point 1 & 1.249 & 1.46 & 1.231 & -1.03 & 1.244 \\
Number & Point 2 & 1.723 & 0.23 & 1.719 & 0.12 & 1.717 \\
& EI exit & 1.982 & 0.05 & 1.981 & 0.04 & 1.980 \\
\hline$\dot{m}(\mathrm{~kg} / \mathrm{s})$ & EI exit & 171.7 & -0.12 & 171.9 & -0.05 & 172.0 \\
\hline
\end{tabular}

Table 5.3: Grid convergence summary.

Table 5.3 lists the values of the selected variables at the selected locations. These variables and locations are the same as the ones used for time convergence verification in the previous section (Figure 5.6). This data is also plotted in Figure 5.9 for visual purposes. As seen from both Table 5.3 and Figure 5.9 the values do not differ significantly when the mesh size is doubled or quadrupled with most of the changes being under $1 \%$. The exception to this is total pressure and Mach number at Point 1 , which change by $2.0 \%$ and $1.46 \%$ when the mesh is doubled and $1.77 \%$ and 1.03 7. when the mesh is doubled again, though the changes are fluctuating around the same value of $53 \mathrm{kPa}$ and 1.24 respectively. Considering that there are no appreciable changes in the values of interest when the number of elements in the mesh is doubled or quadrupled the "Coarse" mesh is deemed to be sufficient in terms of accuracy and is therefore used for all simulations. The results of these simulations are presented in the next section. 


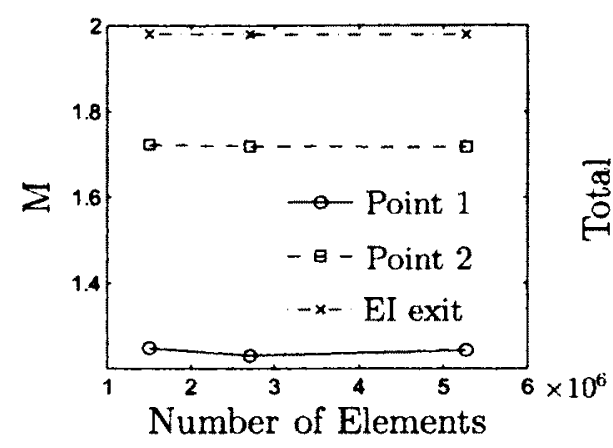

(a) Mach Number

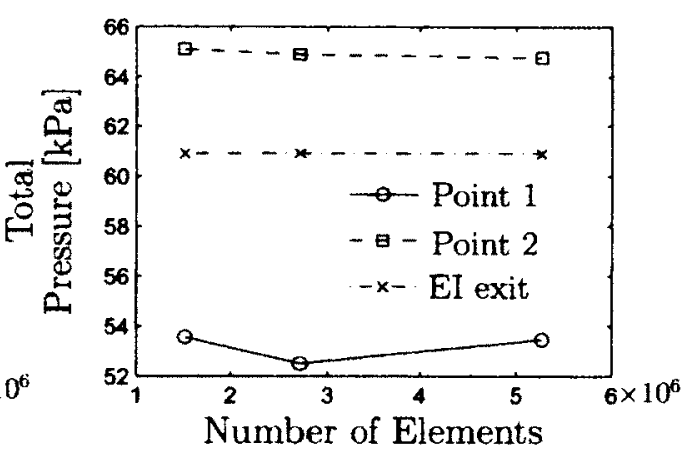

(b) Total Pressure

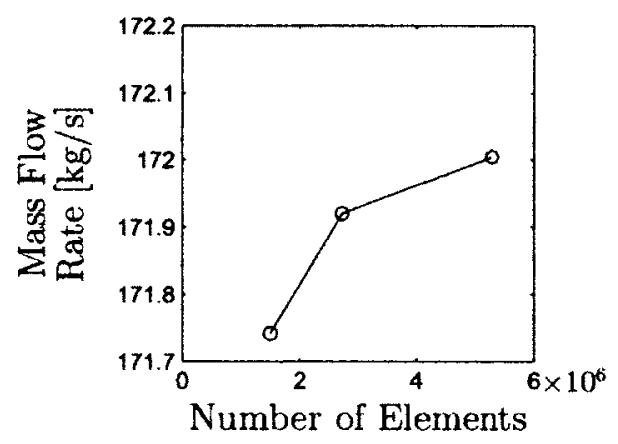

(c) Mass flow rate

Figure 5.9: Grid Convergence.

\subsection{Results and comparison}

\subsubsection{Shock geometry}

Figure 5.10 shows the 3D nature of the shock shapes as approximated from the CFD simulations for different Mach numbers. The shork geometries shown in the figure were obtained by plotting iso-surfaces of the Mach number which is lower than the oncoming Mach number and higher than the Mach number downstream of the shock. As seen from the figure the shocks are relatively simple for $M_{\infty}<3.0$ (Figure $5.10 \mathrm{a}$ and b), however, for $M_{\infty} \geq 3.0$ (Figure $5.10 \mathrm{c}$ and d) the shocks start to intersect making the overall flow field more complex. For all cases the centre body shock (A) closely resembles a cone shock. Figure 5.10a shows the shock geometry to be fairly simple for $M_{\infty}=1.3$ with fairing detached shock (B1) being curved in front of the 


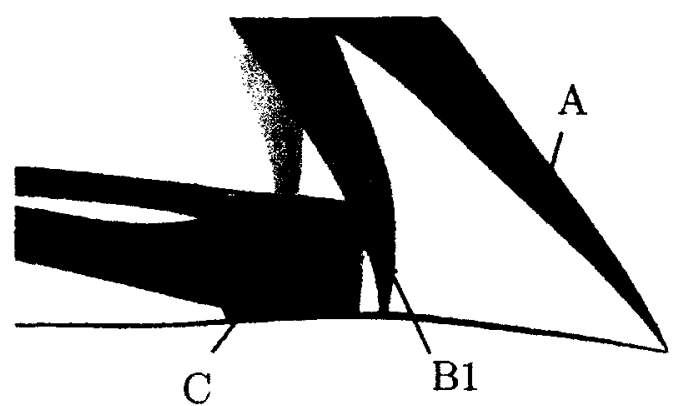

(a) $M_{\infty}=1.3$

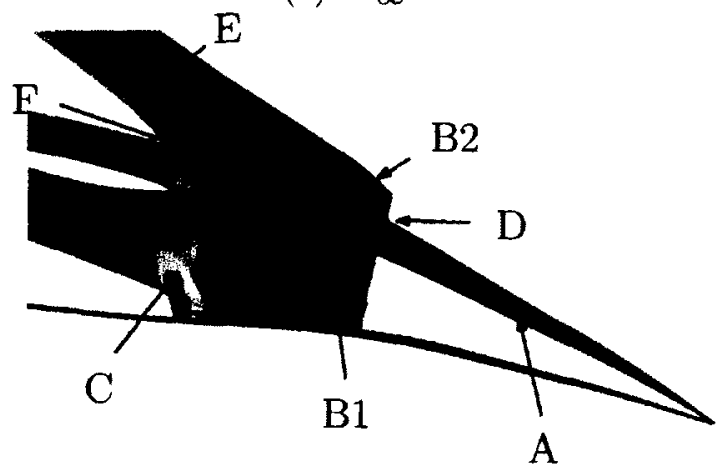

(c) $M_{\infty}=3.0$

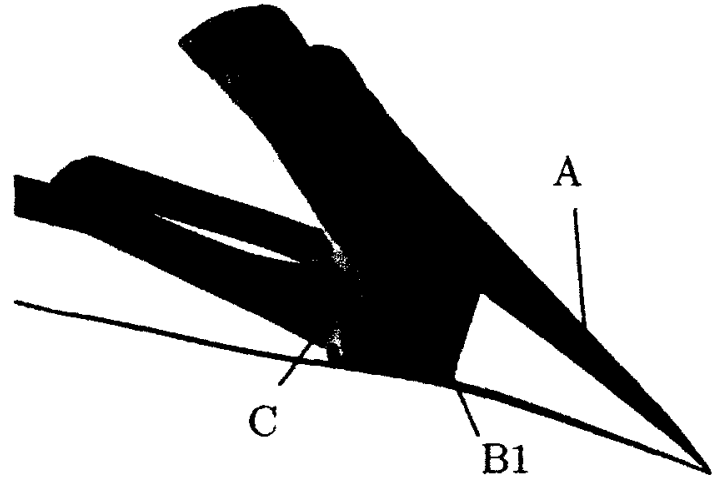

(b) $M_{\infty}=2.0$

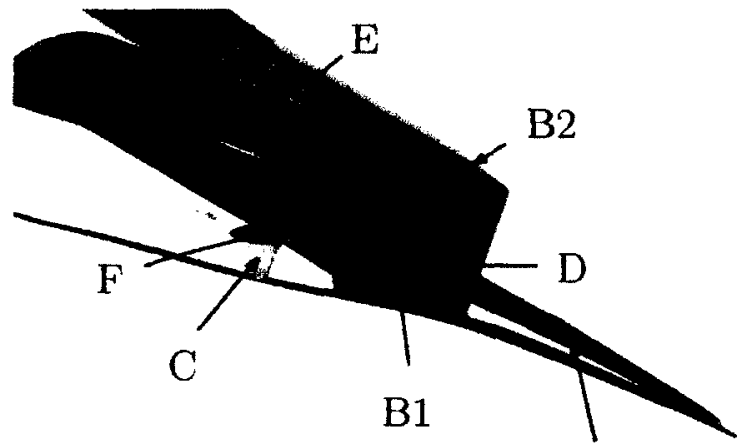

A

Figure 5.10: Shock geometries obtained from 3D CFD simulations: A - Centre-body shock; B1 - Fairing shock; B2 - Fairing shock exposed to free stream; C - Cowl shock; D - Centre body and Fairing Shocks Intersection; E - Cowl and Fairing Shocks Intersection; F - Cowl and Centre body Shocks Intersection

fairing. The Cowl shock (C) is almost normal for the intake region and is a number of cowl radii away from the cowl itself, indicating that the Mach number in this region is relatively low, though still supersonic. As the Mach number increases the shock angles are becoming steeper and the shock stand-off distance becomes smaller for all detached shocks. In the case of $M_{\infty}=2.0$ (Figure $5.10 \mathrm{~b}$ ) the fairing shock (B1) comes off of the fairing at sharper angle, while the cowl shock is now much closer to the cowl, though still normal to the flow. The three shocks seem to intersect well away from the EI geometry. However, for the case of $M_{\infty}=3.0$ the centre body 
shock intersects the fairing and the fairing shock ( $D$ in Figure 5.10c), meaning that part of the fairing is now exposed to the undisturbed free-stream flow (B2) resulting in a stronger shock than the case where the flow passes through the centre body shock before encountering the fairing (B1). For $M_{\infty}=3.0$ both the fairing shock and the centre body shock intersect the cowl shock close to the cowl ( $E$ and F), though at this point the intersection is still outside the EI and has no effect on the flow through the EI. The cowl shock itself becomes less of a normal shock and the Mach stem at the lower section of the shock becomes a more distinguished feature. At $M_{\infty}=7.0$ (Figure 5.10d) the portion of the fairing exposed to the free stream conditions is increased (B2), while the intersection of the centre body and the fairing shocks with the cowl shock happens inside the EI (E and F in Figure 5.10d) resulting in a somewhat complicated shock structure of the cowl shock, a close up of which is shown in Figure 5.11.

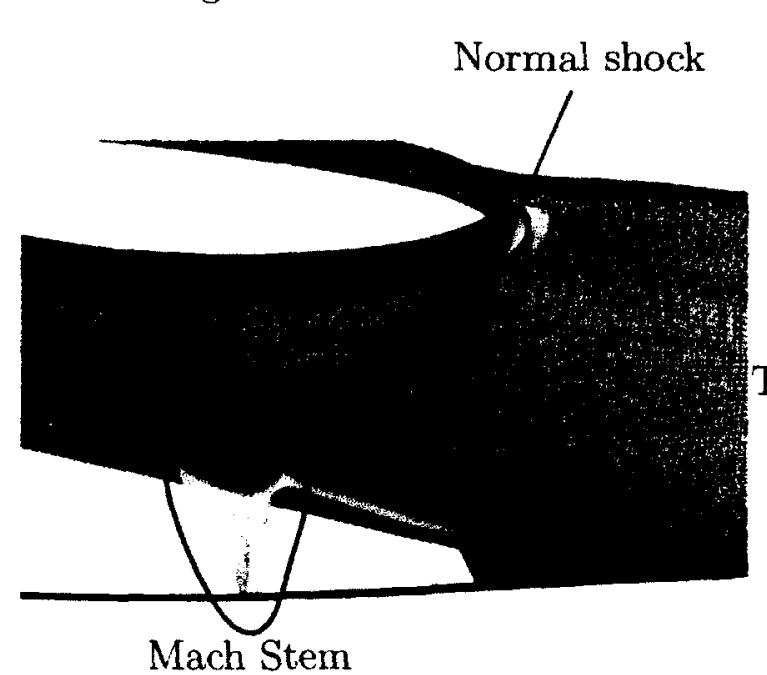

(a)

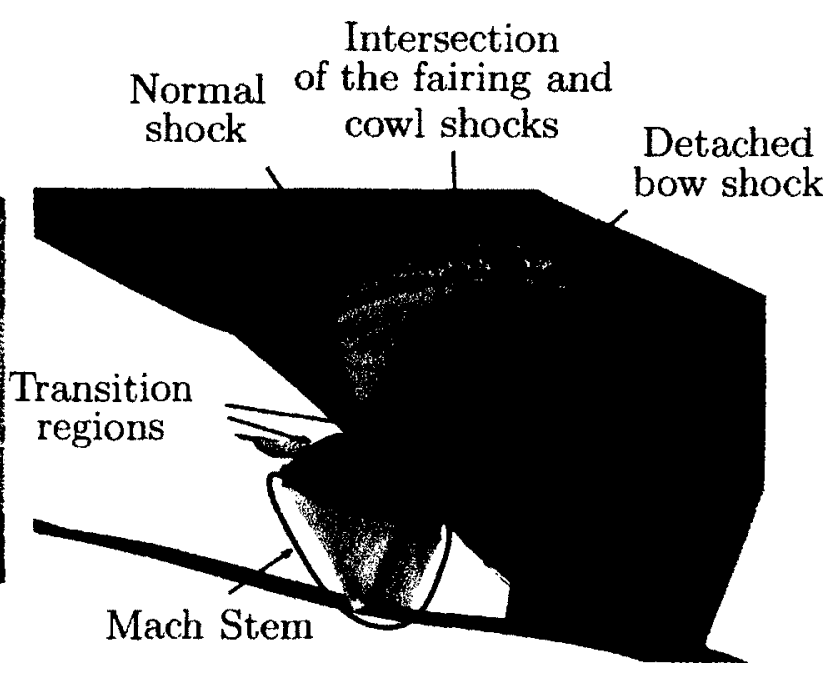

(b)

Figure 5.11: Cowl shock $\left(M_{\infty}=7\right)$.

As seen from Figure 5.11 the cowl shock geometry at $M_{\infty}=7.0$ is quite complicated. A portion of the shock is exposed to the free-stream conditions, which results in two "transition" regions surrounding this normal shock and connecting it to the 
bow shock region and the Mach Stem. The bow shock region is similar to the ones present at lower Mach numbers (Figure 5.10). The two transition regions, however, are at a much sharper angle to the flow resulting in lower changes of properties across these sections. The normal section and these transition regions are not modelled in the estimation method. Instead the cowl shock is assumed to be a $2 \mathrm{D}$ bow shock with Mach stem at the centre body surface.

Figure 5.12 shows the Mach distribution along "Symmetry Plane 1" (shown in Figure 5.1), with estimated shock geometries indicated by thick lines. From the figure the most distinguished features are the three shocks $(A, B$ and $C)$, and the regions of accelerated flow at the bases of both fairing (region D) and cowl shocks (region E). The acceleration is due to the turning of the centre body in the case of the region before the fairing shock (D), and due to both the centre body and the fairing shapes in the region before the cowl shock $(E)$. The three visible shocks consist of the cone shock produced by the centre body $(A)$, the projection of the detached bow shock produced by the fairing (B), and the detached cowl shock (C), with Mach stem (F) visible in the location closer to the centre-body. The projection of the fairing shock is shown in more detail in Figure 5.13, where the fairing shock is coming off the fairing and intersects Symmetry Plane 1 at different downstream locations depending on the radius at which the fairing shock is being considered. Figure 5.13 also indicates a region of interest for further fairing shock analysis with shock planes locations indicated.

In terms of the shock generated by the centre body (A) the estimations and CFD simulations agree reasonably well based on Figure 5.12. As can be seen, with an increase of the Mach number the centre body shock becomes sharper. The projection of the fairing shock also agrees reasonably well between the estimation method and the CFD simulations over the considered range of the Mach numbers. Figure 5.14 shows the Mach distribution on planes normal to the fairing with the estimated shocks 


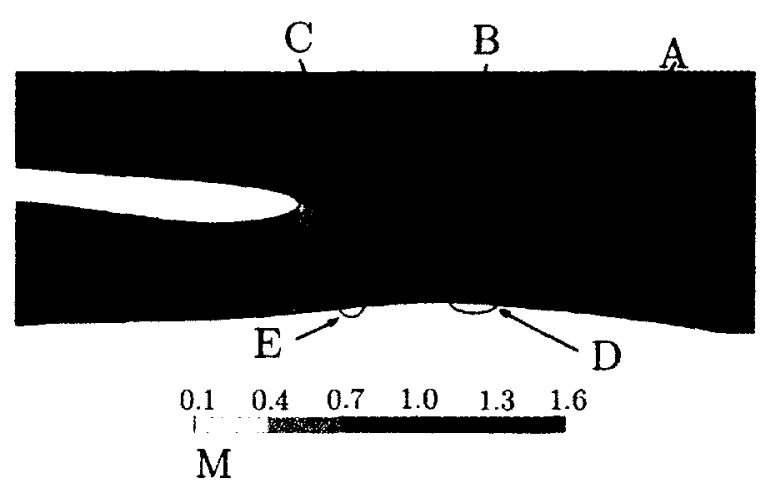

(a) $M_{\infty}=1.3$

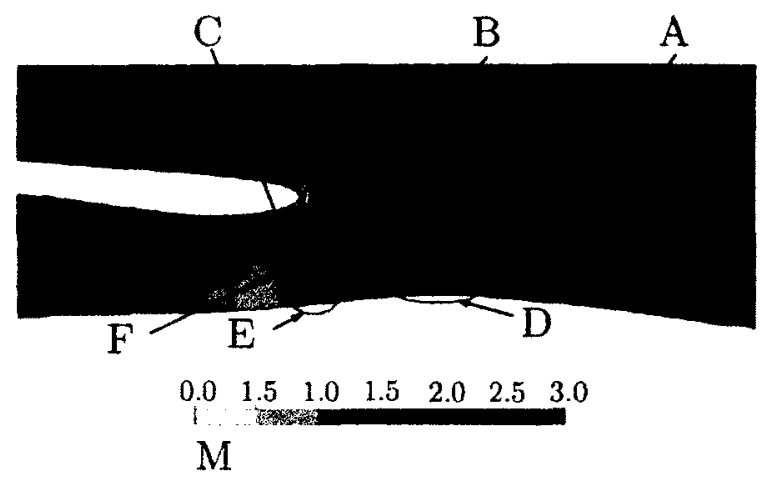

(c) $M_{\infty}=3.0$

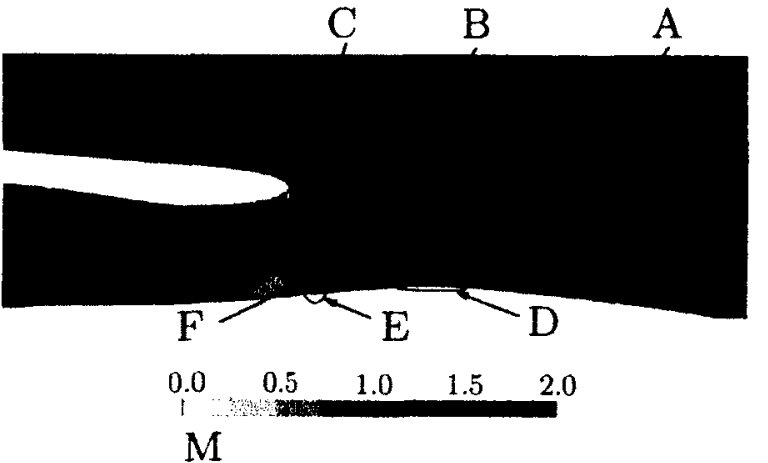

(b) $M_{\infty}=2.0$

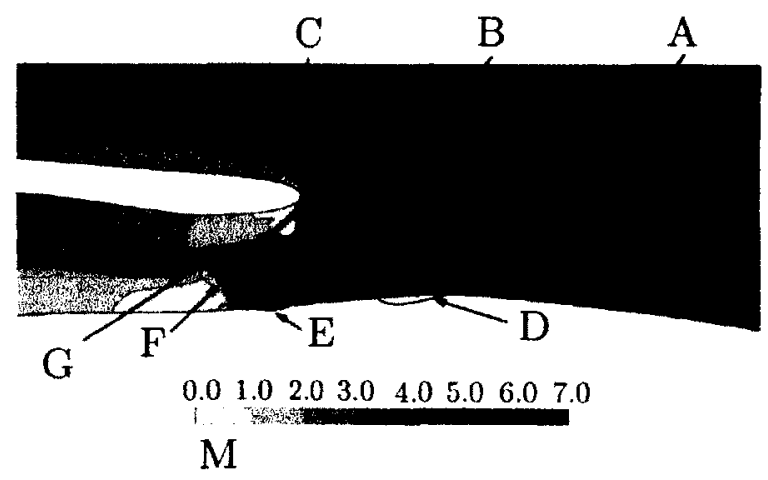

(d) $M_{\infty}=7.0$

Figure 5.12: Mach distribution (Symmetry Plane 1) with lines representing the estimated shocks: A - Centre Body shock, B - Fairing shock, C - Cowl shock, D Acceleration of the flow due to centre body before fairing shock, E - Acceleration of the flow due to the fairing and centre body curvature before the cowl shock, F - Mach Stem, G - Region of high Mach number after cowl shock .

indicated by thick lines. This figure makes it easier to visualise the fairing shock and its projection (B) onto the symmetry plane shown in Figure 5.12. As seen from Figure 5.14 a for $M_{\infty}=1.3$ the location of the estimated fairing shock slightly differs from that calculated by the CFD simulations, though the shape of the shock is predicted quite well. Of note is that according to the simulation the flow is fully subsonic right behind this shock, which is then re-accelerated along the fairing to approximately Mach 1.2-1.3 (E in Figure 5.12). This acceleration of the flow by the fairing is not modelled in the estimation method for the cases where the flow right after the fairing is subsonic (which is the case for $M_{\infty}=1.3$ ) and as such the estimation method 


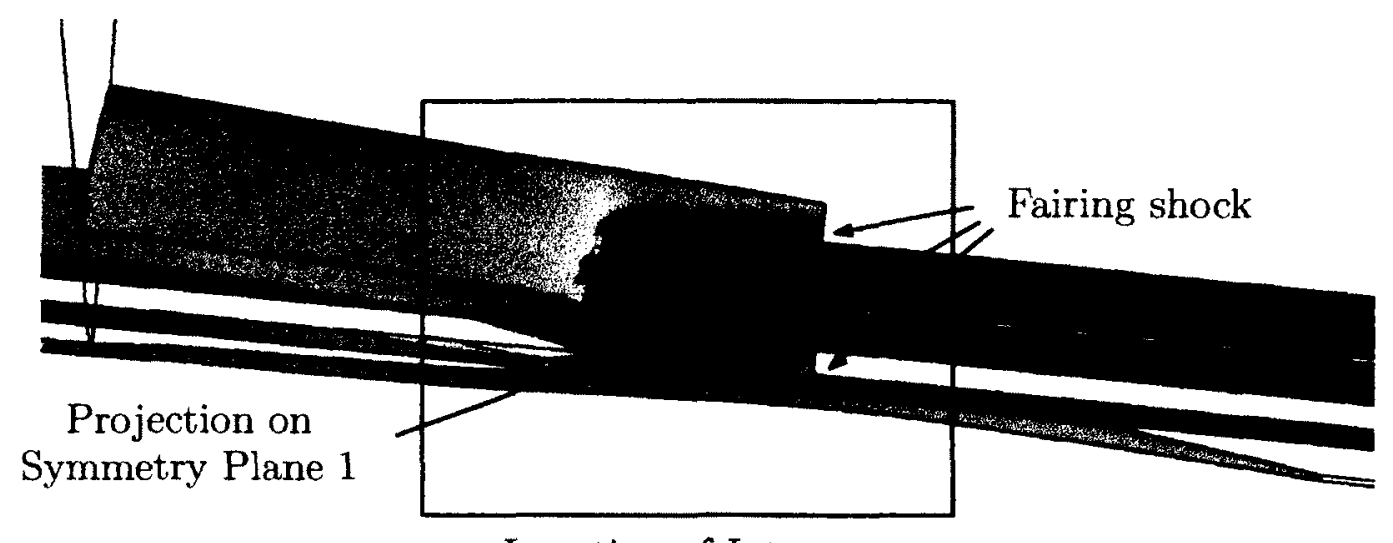

Location of Interest

(Figure 5.14)

Figure 5.13: Fairing shock location and its projection to Symmetry Plane 1.

does not compute the cowl shock (hence the absence of a thick line for cowl shock in Figure 5.14a). The cowl shock, however, is computed for cases where the flow is supersonic after the fairing shock, as shown in the results for $M_{\infty}=2.0$ (Figure $5.12 \mathrm{~b}$ and $5.14 \mathrm{~b}$ ). In this case the cowl shock is estimated as a detached bow shock, while the CFD results show a Mach stem right after the region E. At $M_{\infty}=2.0$ the geometry of the fairing shock follows the one computed by the CFD simulation very closely in both the shock location and its shape. The same can be said about the fairing shock shapes at $M_{\infty}=3.0$ (Figure 5.12c and 5.14c) and $M_{\infty}=7.0$ (Figure $5.12 \mathrm{~d}$ and $5.14 \mathrm{~d}$ ). The Mach stem for these two Mach numbers is now also computed by the estimation method, though in the case of $M_{\infty}=3.0$ the size of the Mach stem is underestimated and it is located further downstream when compared to the CFD results (Figure 5.14c). As was mentioned earlier, at $M_{\infty}=7.0$ both the centre body shock and the fairing shock intersect the cowl shock producing a complicated cowl shock geometry (Figure 5.11). Despite this, the estimation method is able to correctly calculate the size of the Mach stem as well as the overall cowl shock shape at Symmetry Plane 1 (Figure 5.12d).

Figure 5.12 also shows an acceleration of the flow along the centre body (D). As 


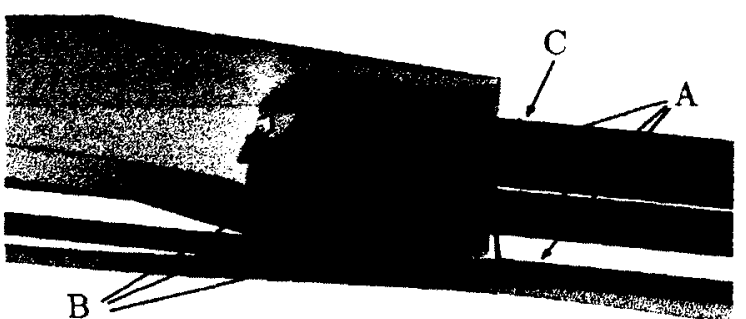

$0.10 \quad 0.25,0.40 \quad 0.55 \quad 0.70 \quad 0.85 \quad 1.00 \quad 1.15 \quad 1.30$

M

(a) $M_{\infty}=1.3$

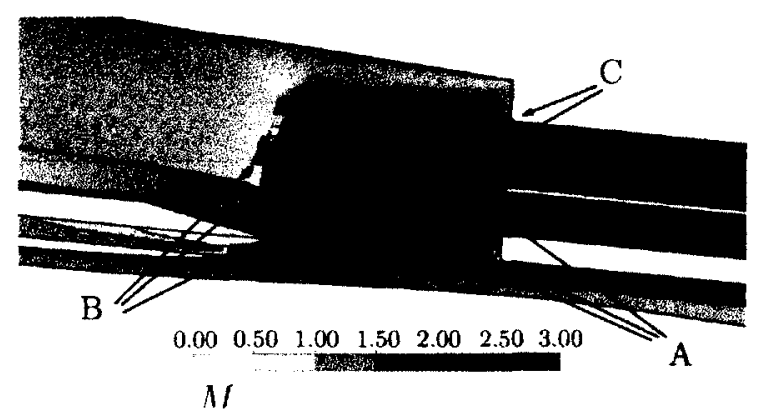

(c) $M_{\infty}=3.0$

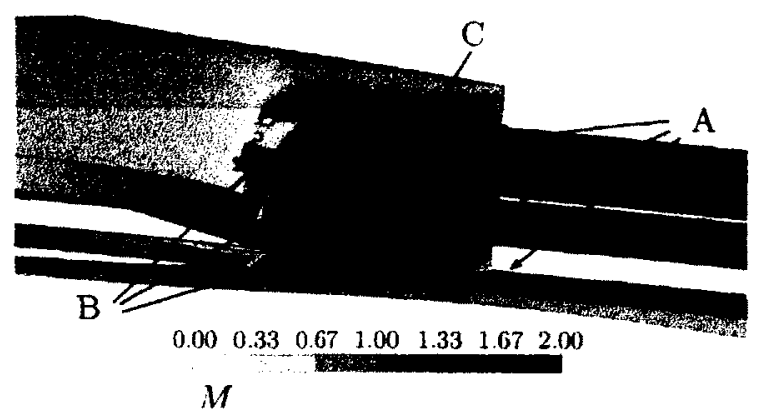

(b) $M_{\infty}=2.0$

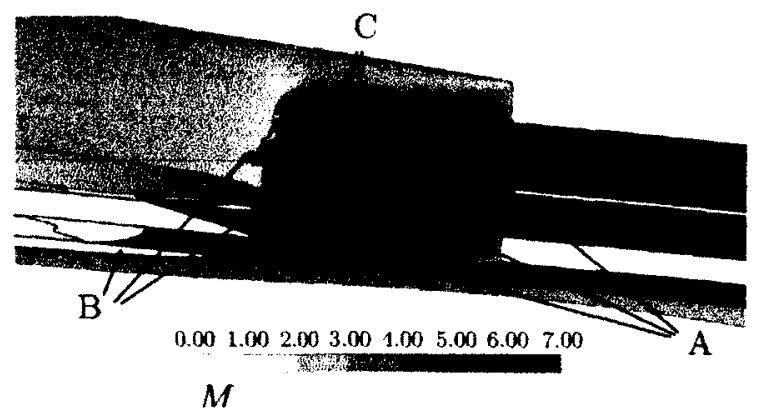

(d) $M_{\infty}=7.0$

Figure 5.14: Mach distribution after the fairing shock (Selected Planes): A - Fairing shock, B - Cowl shock, C - Estimated fairing shock and its projection onto the Symmetry Plane 1 (thick lines).

noted in Chapter 3 this acceleration is estimated using the Prandtl-Meyer function along the centre body geometry. A comparison of this effect between the estimations and the CFD results is shown in Figure 5.15. The CFD results are extracted from Symmetry Plane 1 and in the case of the estimation method results are truncated once the fairing shock is reached. After the fairing shock the estimated flow is averaged and a meaningful comparison along the centre body is no longer possible. As seen from the figure despite the fact that the geometry of the nose tip of the centre body was adjusted in the CFD simulations (Section 5.2) the results from the estimation method and the CFD simulations agree with each other reasonably well meaning that the estimation method captures the flow acceleration at the centre body. Figure 5.15 also indicates that the estimated locations of the fairing shock matches that from the 


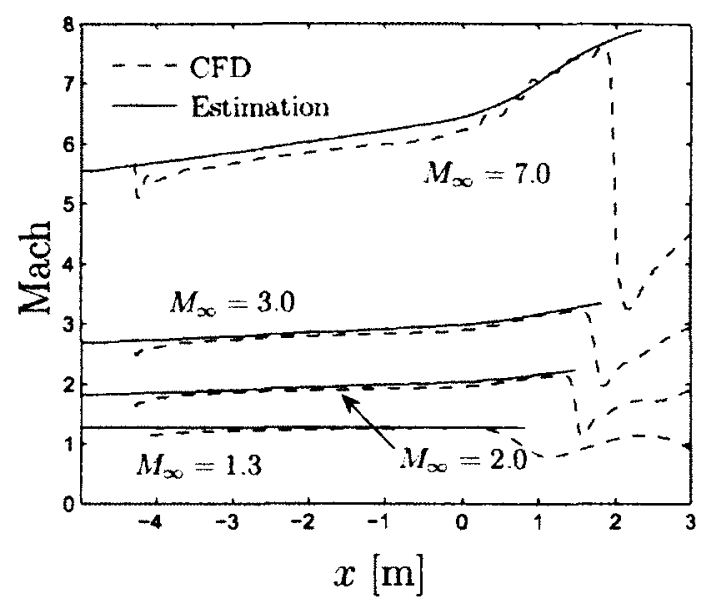

Figure 5.15: Mach number along the centre body surface (estimation line ends at the location of the fairing shock).

CFD results as indicated by the location at which the estimation curve ends and the location of the sharp decrease in Mach number for the CFD curves.

\subsubsection{Total pressure}

Figure 5.16 shows data for the total pressure ratio at three different locations: after the centre body shock (CB shock), after the fairing shock and after the cowl shock (at the EI exit plane). An example of these locations is shown in Figure 5.17. The total

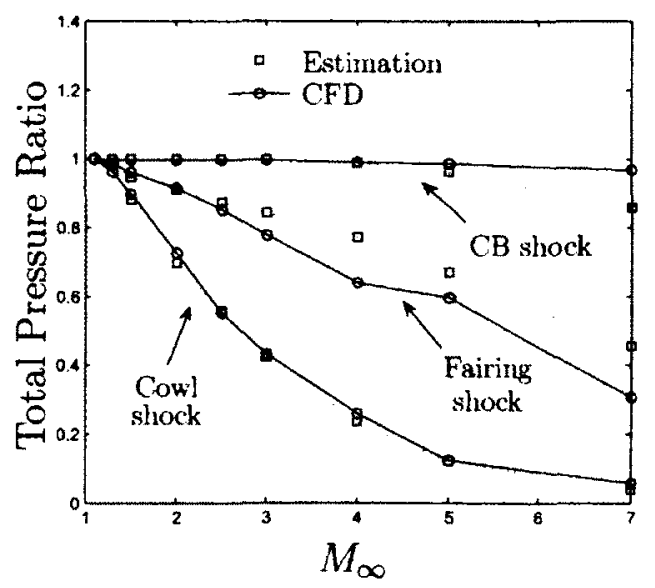

Figure 5.16: Pressure loss through the intake with cumulative total pressure after each shock shown. 
pressure ratio is the averaged total pressure behind a respective shock and normalized with the free stream total pressure. As seen from the figure the total pressure ratio starts to differ after the centre body shock at $\Lambda_{\infty}=5$. This difference comes from the fact that the geometry used for the simulation was slightly changed and as a result the deflection angle seen by the oncoming flow is smaller than in the actual geometry due to the shape of the centre body. This results in a weaker centre body shock calculated by the CFD simulations, and therefore a higher total pressure.

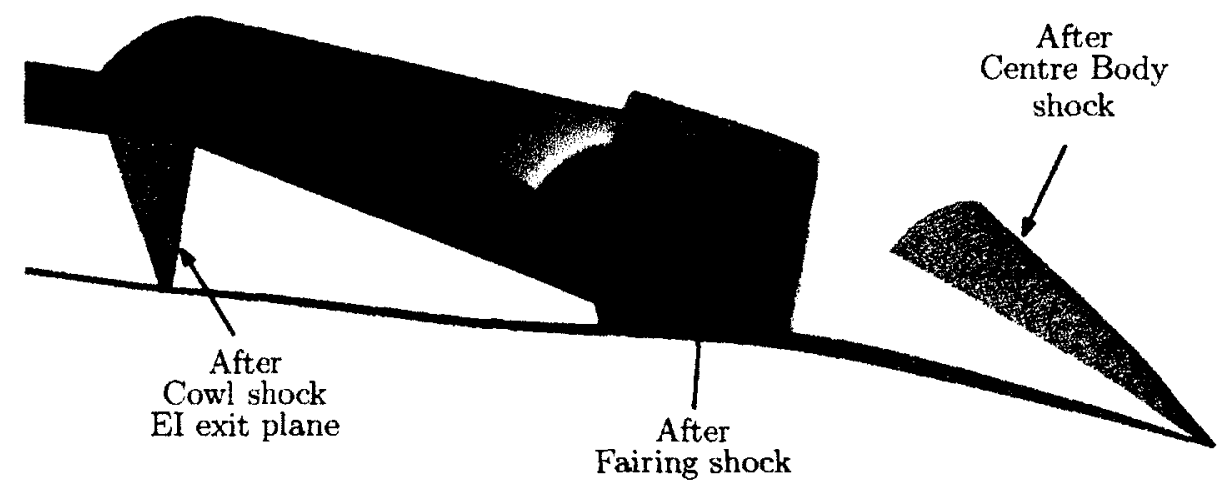

(a) CFD

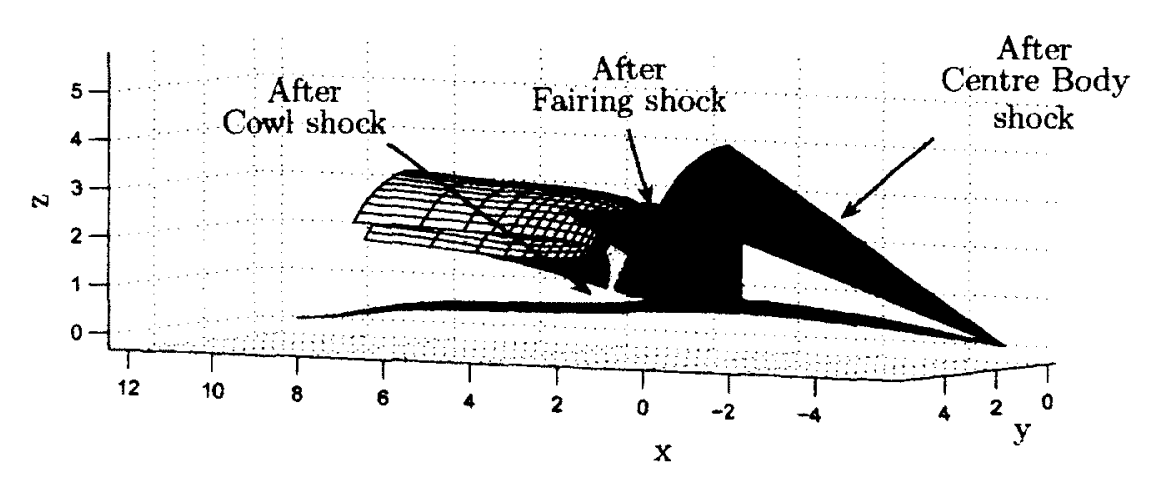

(b) Estimation

Figure 5.17: Example of averaging locations for total pressure comparison $\left(\Lambda \Lambda_{\infty}=\right.$ $2.0)$.

The difference in the fairing shock line is mainly due to different averaging regions. In the case of the simulations, the averaged results were obtained at the surfaces which 
were offset by $0.1 \mathrm{~m}$ from the surface of each respective shock. The averaging of the results was done for the fairing shock truncated by either the topmost fairing location or some distance away from the cowl shock as to avoid shock intersections (Figure $5.17 a)$. In the case of the estimation method the averaging was performed across the entice estimated shock surface, which is calculated from the bottom to the top of the fairing leading edge and was not bound by shock intersections (Figure 5.17b). This means that the estimated averaging produces a higher total pressure after the fairing shock as it covers the region further away from the fairing, where the total pressure drop across the shock is lower (the shock angle in this region is sharper). Even though this is the case, the trend for the estimated fairing shock is the same as the one calculated with CFD simulations.

In terms of the cowl shock line the estimated total pressure follows the results from the CFD simulations very well. The total pressure averaging, however, is performed at different locations. In the case of the estimation method the averaging is done right after the cowl shock and the flow is assumed isentropic afterwards meaning that the total pressure at the exit plane of the EI is the same as right after the cowl shock. In the case of the CFD simulations the averaging is performed at the EI exit plane because the cowl shock becomes harder to isolate for this purpose. On average for $M_{\infty} \leq 5.0$ the difference in total pressure between the estimations and the CFD simulation results does not exceed 3\%. As seen from Figure 5.18 and Table 5.4 the difference is below $10 \%$ for all cases. The only exception to this is $M_{\infty}=7.0$ for which the difference increases to $30 \%$. The possible reason for the large difference

\begin{tabular}{|c|c|c|c|c|c|c|c|c|c|}
\hline Mach Number & 1.1 & 1.3 & 1.5 & 2.0 & 2.5 & 3.0 & 4.0 & 5.0 & 7.0 \\
\% difference & -0.4 & 2.0 & -1.5 & -3.9 & 1.0 & -1.4 & -7.9 & -1.0 & -30.6 \\
\hline
\end{tabular}

Table 5.4: Pressure ratio difference.

between the computed total pressure at $M_{\infty}=7.0$ is the complex shock structure 


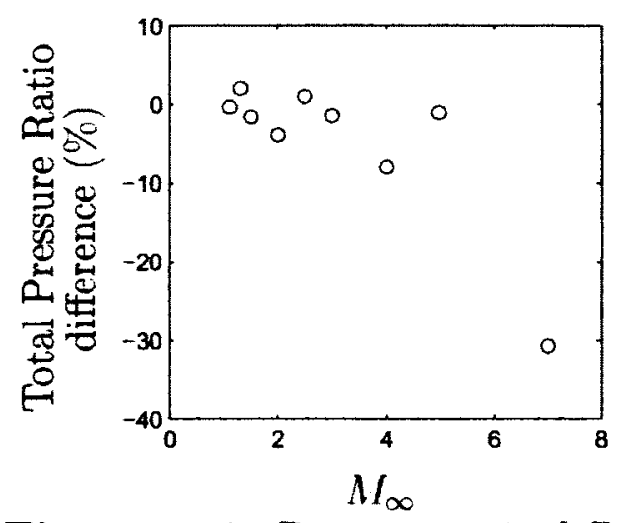

Figure 5.18: Pressure ratio difference.

for the cowl shock. As shown in Figure 5.12d there is a region (G) of the flow that doesn't change the Mach number significantly while passing through the shock (just above the Mach stem and region E). This indicates that the shock in this region is very weak and corresponds to one of the transition regions shown in Figure 5.11b which is not modelled in the estimation method.

This analysis indicates that the estimation method at least for this geometry was able to perform very good as compared to the full 3D CFD simulations when calculating the total pressure drop across the EI while taking less than 3 seconds per flight condition on $3.0 \mathrm{GHz}$ processor as compared to days on a system with 8 cores at $2.4 \mathrm{GHz}$. The analysis of the nuass flow rate adds additional support to this statement.

\subsubsection{Mass flow rate}

Figure 5.19 shows the two mass flow rate estimations described in Chapter 3 (Choked and Total-Pressure-Mach based calculations) used to determine the mass flow rate through the EI. Based on the figure the flow is choked up to and including $M_{\infty}=2.0$ and is unchoked afterwards, which is also supported by Figure 5.12 where the flow is fully subsonic after the cowl shock along the symmetry plane for cases of $M_{\infty}=1.3$ 
and $M_{\infty}=2.0$ while there are supersonic regions observed in the other two Mach number cases considered.

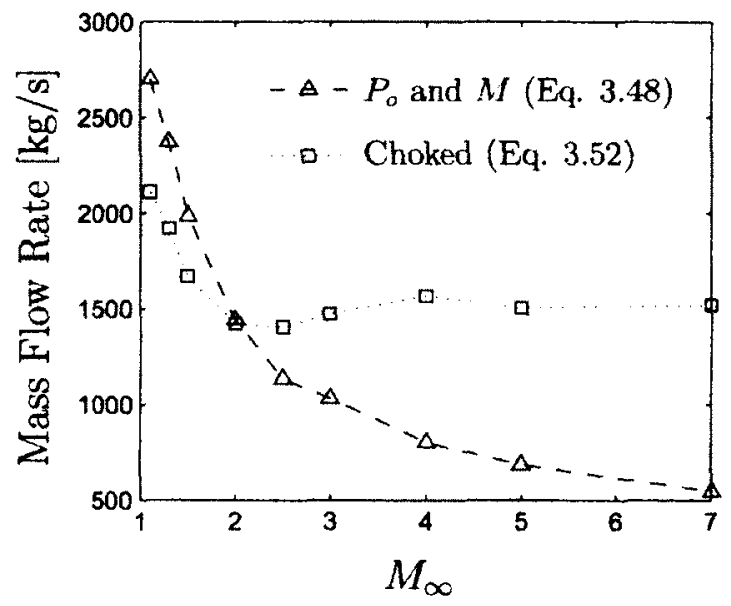

Figure 5.19: Estimations of the mass flow rate.

Figure 5.20 shows estimated mass flow rate comparison to the CFD results. As seen from the figure the estimation has a good agreement with the CFD simulations. Figure 5.21 and Table 5.5 show that the difference between the two does not exceed $10 \%$. The estimation method seem to overpredict the mass flow rate for choked conditions by about $5 \%\left(M_{\infty} \leq 2.0\right)$ and underpredict it for the unchoked conditions on average by about $5 \%\left(M_{\infty}>2.0\right)$. The notable outlier to this is at $M_{\infty}=7.0$ where the estimation overpredics the mass flow rate.

\begin{tabular}{|c|c|c|c|c|c|c|c|c|c|}
\hline Mach Number & 1.1 & 1.3 & 1.5 & 2.0 & 2.5 & 3.0 & 4.0 & 5.0 & 7.0 \\
\% difference & 3.7 & 6.2 & 3.3 & 3.5 & -5.0 & -3.7 & -8.6 & -5.1 & 4.6 \\
\hline
\end{tabular}

Table 5.5: Mass flow rate percent difference. 


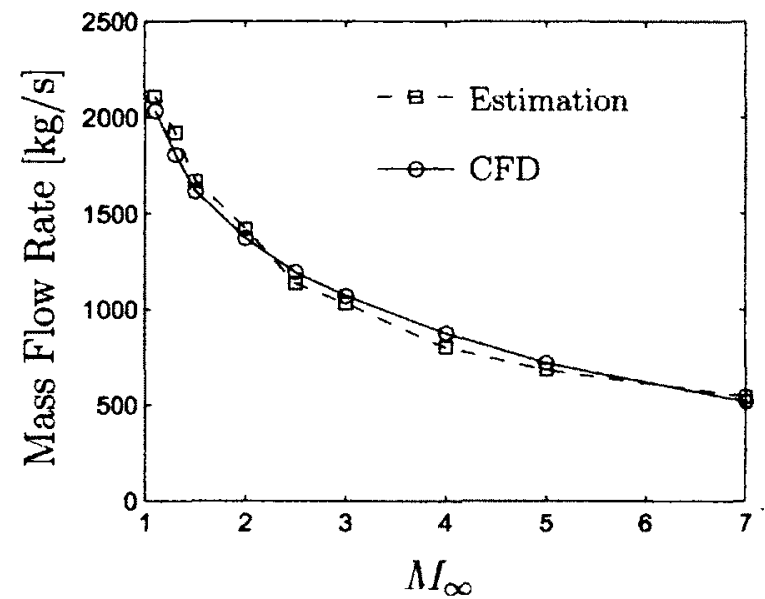

Figure 5.20: Mass flow rate comparison.

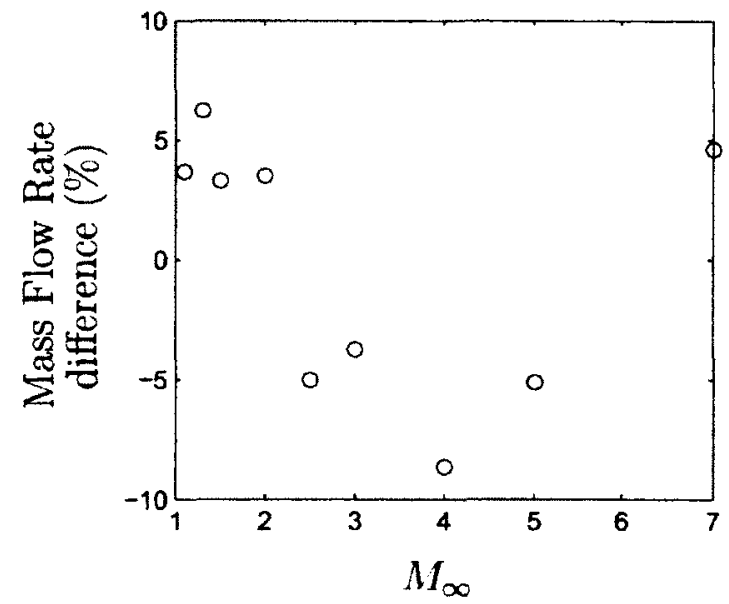

Figure 5.21: Mass flow rate percent difference. 


\section{Chapter 6}

\section{Conclusions and Recommendations}

\subsection{Conclusions}

The performance of a Rocket-Based-Combined-Cycle Exchange Inlet design is analysed at supersonic conditions by the developed estimation method. The results of this method are compared to commercially available CFD solver (Ansys-CFX). The analysed geometry produces three sets of shocks, which could be approximated by a cone shock and a series of detached bow shocks. The estimation method uses these shock approximations to find the shock geometries produced by the Exchange Inlet geometry, and, based on these shock geometries, calculates the total pressure drop across the entire Exchange Inlet geometry using analytical solutions for oblique shocks. The comparison of the estimations to the CFD results shows a good agreement. For example, the total pressure at the exit plane of the exchange inlet differs on average by less than $3 \%$ for Mach numbers below 5.0 and by $30 \%$ for Mach 7.0. The agreement is also very good for the mass flow rate through the Exchange Inlet which is on average less than $5 \%$ and the maximum difference does not exceed 10 $\%$ for any of the tested conditions (including Mach 7.0). The major sources for the difference between the CFD results and those produced by the estimation method are attributed to the complexity of the cowl shock and the interaction between the cowl 
and the fairing shocks which is especially true for the Mach 7.0 case, hence the large difference.

The estimation code completes a single flight condition computation in just under 3 seconds on a $3.0 \mathrm{GHz}$ processor which is significantly faster than the transient CFD simulations. In addition to that the estimation method provides with reasonable results and as such it could be further used within an Exchange Inlet geometry optimization algorithm.

\subsection{Recommendations}

The analysis of the Exchange Inlet geometry with both estimation method and CFD simulations showed a few shortcomings of the Exchange Inlet at supersonic speeds. The following are the recommendations for improvements in the Exchange Inlet geometry:

- The acceleration of the flow before shocks should be avoided and, if possible, the flow should be decelerated. For supersonic flow the acceleration is caused by expansion around concave surfaces, while deceleration is caused by convex surfaces. The concave surfaces before the fairing and the cowl should be avoided, or the curvature of these surfaces should be kept at a minimum.

- The leading edges should be sharp for the supersonic flow. The blunt leading edges of the fairings and the cowl geometries cause detached bow shocks which have a very strong shock section which in turn results in higher total pressure drop across the Exchange Inlet. The size of this strong shock section is linearly proportional to the radius of the leading edge, meaning the radius of the leading edge of the fairings and the cowl should be kept to a minimum.

- The positioning of the fairing with respect to the cowl is not justified from 
a supersonic perspective. Positioning the fairings fully within the cowl could prevent the fairing shock from occurring, provided the flow is kept at subsonic speed after the cowl.

The following is the list of recommendations for future work to improve the estimation method:

- The testing of the performance of the method for different Exchange Inlet geometries needs to be performed to verify the robustness of the estimation method.

- The code would require additional modification should the fairing be moved behind the cowl leading edge. The current version of the estimation method stops once the properties after the cowl shock are computed. To allow for fairing to be moved behind the cowl the flow field after the cowl shock would need to be estimated as well. 


\section{List of References}

[1] "Specific impulse." Accessed December 03, 2012. from littp://en.wikipedia.org/wiki/Specific_impulse.

[2] R. Daines and C. Segal. "Combined rocket and airbreathing propulsion systems for space-launch applications." Journal of Propulsion and Power 14(5), "605612" (1998).

[3] S. A. Whitmore and B. J. Dunbar. "Orbital space plane: Past, present, and future." AIAA 2003-2718 (2003).

[4] V. Lukashevich. "Bor -1, -2, -3." Retrieved September 15, 2012, from http://www.buran.ru/htm/bors.htm (Russian).

[5] V. Lukashevich and I. Afanasjev. Kosmicheskie Krilja (Space Wings). LenTa Stranstvij, Moscow, Russia (2009).

[6] J. Ray. "Pegasus launch report." Accessed December 03, 2012, from http://spaceflightnow.com/pegasus/hete2/status.html (2000).

[7] W. J. Escher. "Synerjet for earth/orbit propulsion - revisiting the 1966 nasa/marquardt composite (airbreathing/rocket) propulsion system study (nas7377)." AIAA 96-3040 (1996).

[8] Y. M. Timnat. "Recent developments in ramjets, ducted rockets and scramjets." Progress in Aerospace Science 27, 201-235 (1990).

[9] R. S. Fry. "A century of ramjet propulsion technology evolution." Journal of Propulsion and Power 20(1) (2004).

[10] R. Longstaff and A. Bond. "The skylon project." AIAA 2011-2244 (2011).

[11] R. Daines and M. Bulman. "Computational analyses of dynamic rocket ejector flowfields." AIAA 96-2686 (1996). 
[12] J. D. Escher and R. E. Schnurstein. "A retrospective on early cryogenic primary rocket subsystem designs as integrated into rocket-based combined-cycle (rbcc) engines." Presented at the $29^{\text {th }}$ Joint Propulsion Conference (Jul, 1993).

[13] F. Dykstra, A. Maree, M. Caporicci, and H. Immich. "Experimental investigation of the thrust enhancement potential of ejector rockets." AIAA 97-2756 (97).

[14] A. Seebass, S. Murthy, and E. Curran. "High speed flight propulsion systems." Progress in Astronautics and Aeronautics 137 (1991).

[15] J. J. Bertin. Hypersonic Aerothermodynamics. AIAA, Washington, USA (1994).

[16] "Gnom." Accessed December 03, 2012, from http://astronautix.com/lvs/gnom.htm.

[17] "Nasa's x-43a scramjet breaks speed record." Accessed December 03, 2012, from http://www.nasa.gov/missions/research/x43_schedule.html (2004).

[18] D. J. Cerantola and J. Etele. "A nozzle concept to entrain atmospheric air for ejector operation." AIAA 2006-8089. Presented at the 14th AIAA/AHI Space Planes and Hypersonic Systems and Technologies Conference. (2011).

[19] D. Cerantola. Rocket Nozzle Design with Ejector Effect Potential. Master's thesis, Carleton University (2007).

[20] T. Waung and J. Etele. "An ejector air intake design method for a novel rbcc rocket nozzle." AIAA (2009).

[21] T. Yuen and J. Etele. "Exchange inlet design for enhanced rbcc rocket-air mixing." AIAA (2011).

[22] G. Chorkawy. Exchange Inlet Design Optimization by Genetic Algorithm. Master's thesis, Carleton University (2011).

[23] ANSYS ${ }^{\circledR}$,Inc. ANSYS ${ }^{\circledR}$ ICEM CFD/AI Environment, Release 12.0 Documentation (2009).

[24] ANSYS ${ }^{\circledR}$,Inc. ANSYS ${ }^{\circledR}$ CFX, Release 12.0 Documentation (2009).

[25] W. Hui, J. Hu, and G. Zhao. "Gridless computation using the unified coordinates." (2004).

[26] J. Anderson. Modern Compressible Flow. McGraw-Hill Publishing Company (1990). 
[27] A. Ambrosio and A. Wortman. "Stagnation point shock detachment distance for flow around spheres and cylinders." American Rocket Society Journal 32, 281 (1962).

[28] F. S. Billig. "Shock-wave shapes around spherical \& cylindrical-nosed bodies." Journal of Spacecraft and Rockets 4, 822-823 (1967).

[29] E. S. Love. "A reexamination of the use of simple concepts for predicting the shape and location of detached shock waves." National Advisory Committee for Aeronautics NACA TN4170 (1957).

[30] G. Moretti and M. Abbett. "A time dependent computational method for blunt body flows." AIAA 4, 2136-2141 (1966).

[31] The MathWorks ,Inc. MATLAB, R2011b (2011).

[32] F. M. White. Fluid Mechanics. McGraw-Hill Publishing Company (1999).

[33] J. Anderson. Hypersonic and High Temperature Gas Dynamics. McGraw-Hill Book Company (1989).

[34] E. H. Hirschel. Basic of Aerothermodynamics. Springer-Verlag, Germany (2005).

[35] M. J. Zucrow and J. D. Hoffinan. Gas Dynamics: Multidimensional flow. John Wiley and Sons, New York, USA (1976).

[36] G. Emanuel. Gasdynamics: Theory and Applications. AIAA, New York, USA (1986).

[37] H. Versteeg and W. Malalasekera. An Introduction to Computational Fluid Dynamics: The Finite Volume Method. Pearson Education Limited (2007).

[38] G. Ben-Dor and K. Takayama. "The influence of the downstream pressure on the shock wave reflection phenomenon in steady flows." Journal of Fluid Mechanics 386, 213-232 (1999).

[39] C. Moutan. Transition between Regular Reflection and Mach Reflection in the Dual-Solution Domain. Ph.D. thesis, California Institute of Technology (2007).

[40] H. Liepmann and A. Roshko. Elements of Gasdynamics. Galcit aeronautical series. John Wiley \& Sons (1957).

[41] "Earth atmosphere model - metric units." Http://www.grc.nasa.gov/WWW/K12/airplane/atmosmet.html. 
[42] D. Andreadis. "Scramjet engines enabling the seamless integration of air and space operations." Retrieved April 10, 2012, from http://www.pwrengineering.com/data.htm (2006). 